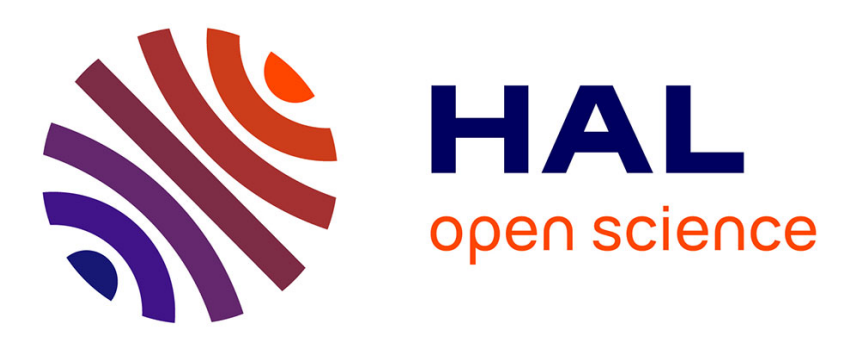

\title{
Rock strength and texture evolution during deformation in the Earth's ductile lithosphere: a two-phase thermodynamics model
}

\author{
Benoit Bevillard, Guillaume Richard, Hugues Raimbourg
}

\section{- To cite this version:}

Benoit Bevillard, Guillaume Richard, Hugues Raimbourg. Rock strength and texture evolution during deformation in the Earth's ductile lithosphere: a two-phase thermodynamics model. Geochemistry, Geophysics, Geosystems, 2019, 20 (2), pp.1095-1137. 10.1029/2018GC007881 . insu-01986113

\section{HAL Id: insu-01986113 https://hal-insu.archives-ouvertes.fr/insu-01986113}

Submitted on 27 Feb 2019

HAL is a multi-disciplinary open access archive for the deposit and dissemination of scientific research documents, whether they are published or not. The documents may come from teaching and research institutions in France or abroad, or from public or private research centers.
L'archive ouverte pluridisciplinaire HAL, est destinée au dépôt et à la diffusion de documents scientifiques de niveau recherche, publiés ou non, émanant des établissements d'enseignement et de recherche français ou étrangers, des laboratoires publics ou privés. 


\section{Geochemistry, Geophysics, Geosystems}

\author{
RESEARCH ARTICLE \\ 10.1029/2018GC007881 \\ Key Points: \\ - The presented model adresses the \\ evolution of mylonite texture during \\ ductile deformation \\ - This model tracks the role of creep \\ mechanisms on the mixing state \\ evolution of the aggregate \\ - An effective viscosity that depends on \\ the mixing degree of the two-phase \\ increases stress weakening
}

Correspondence to:

B. Bevillard,

benoit.bevillard@cnrs-orleans.fr

Citation:

Bevillard, B., Richard, G., \& Raimbourg, H. (2019). Rock strength and texture evolution during deformation in the Earth's ductile lithosphere: A two-phase thermodynamics model. Geochemistry, Geophysics, Geosystems, 20.

https://doi.org/10.1029/2018GC007881

Received 16 AUG 2018 Accepted 10 JAN 2019 Accepted article online 16 JAN 2019

\section{Rock Strength and Texture Evolution During Deformation in the Earth's Ductile Lithosphere: A Two-Phase Thermodynamics Model}

\author{
Benoit Bevillard $^{1}(\mathbb{D})$, Guillaume Richard ${ }^{1}(\mathbb{D})$, and Hugues Raimbourg ${ }^{1}(\mathbb{D}$ \\ ${ }^{1}$ Institut des Sciences de la Terre d'Orléans, UMR 7327 CNRS/Université d'Orléans 1A rue de la Férollerie, Orléans \\ Cedex 2, France
}

\begin{abstract}
Building upon the two-phase and grain damage theory, we propose a new formulation allowing to track the evolution of phase mixing/segregation during ductile deformation of a two-phased aggregate. Our model is based on a set of variables characterizing a rock texture: the mean grain sizes and the phase proportion. During ductile deformation, activation of different micromechanical processes impacts the aggregate texture. Dislocation and diffusion creep are the two main deformation processes considered. We only account for the effect of Zener pinning in slowing down grain growth and allow for active grain-size reduction mechanisms in the diffusion creep domain. For this purpose, an equation is proposed to track the phase mixing evolution during ductile deformation. Numerical application using anorthite rheology shows that any grain reduction mechanisms that could be active in the diffusion creep regime requires a very high partition fraction in order to reach the grain size predicted by the feldspar piezometer. Application of this model to gabbroic composition, relevant for the ductile crust, demonstrates that the strong coupling between phases grain sizes and interface evolution results in steady-state grain sizes far below the field boundary. This effect is coeval with an important increase of mixing between the two phases. In addition, accounting for the phase mixing results in a drop of the global aggregate stress during deformation. This model allows for further comparison of mylonitized textures evolution with natural shear zones at the local and regional scales.
\end{abstract}

Plain Language Summary In the Earth lower crust, the rocks deform by slow creep instead of breaking due to high temperature and pressure. Observations of natural material deformed in such conditions show that deformation concentrates on narrow-banded structures. At small scales $(1 \mathrm{~cm}$ to $1 \mu \mathrm{m}$ ), these shear bands generally present a very fine mean crystal size and a very good mixing of the minerals. These features are characteristic of deformed rocks, but their origins and consequences on geological structures at larger scales $(1 \mathrm{~m}$ to $100 \mathrm{~km}$ ) are yet to be fully understood. In this study we propose a physical model (a mathematical representation of reality) that allows to represent a rock consisting of two minerals, their mean crystal sizes and proportions and the quality of their mixing. This is based on parameters constrained experimentally for each pure mineral. Using this model, we are able to track the evolution of these rocks microscopical characteristic and their consequences on the rock strength during deformation. Eventually, the results allow us to refine our understanding of the implied processes by comparing the computed variables to measures performed on rock samples. The physical equations proposed could then be used to model the rock strength in geodynamical models at larger scales.

\section{Introduction}

Since the development of the theory of plate tectonics, the lithosphere is described as the upper boundary layer of mantle convection. Within this layer, the stress field induced by the motion of convective asthenosphere is mainly accommodated by deformation at plate boundaries. Within the Earth crust, this deformation appears heterogeneously distributed. This is evidenced by large-scale geological structures such as mountain ranges, rifts, oceanic margins, strike-slip faults, or subduction trenches which exhibit intense deformation and bound regions of much weaker strain. While the uppermost part of the crust exhibits brittle deformation, the lower crust and lithospheric mantle are considered to accommodate tectonic stresses through plastic deformation and creep flow (Kohlstedt et al., 1995; Wilks \& Carter, 1990). The rheology of
(C)2019. American Geophysical Union. All Rights Reserved. 
such materials is classically approximated by viscous flow with an effective viscosity depending on the intensive parameters and/or the properties of the material (Kohlstedt, 2007; Nabarro, 1967; Weertman, 1957). Numerous control factors play a role in the rheology of lithospheric rocks.

Deformation experiments (Hirth \& Kolhstedt, 2003; Karato et al., 1986) on olivine aggregates described its rheology as a combination of power-law and Newtonian behavior. In the case of lower crust materials, similar experiments involving plagioclase yielded similar conclusions (Rybacki \& Dresen, 2000, 2004; Tullis et al., 1991). The power-law rheology generally corresponds to dislocation (grain size insensitive) creep where the material undergoes dynamic recrystallization and grain-size reduction. On the other hand, at lower strain rate or higher temperature, the deformation is dominated by diffusion (grain size sensitive) creep, and the material displays a Newtonian behavior. These studies provided empirical flow laws which remain an essential tool in order to understand the ductile behavior of the rocks composing the lithosphere. Figure E1 displays a stress/grain-size deformation map for anorthite (Rybacki \& Dresen, 2000), illustrating the rheology resulting from a combination of these two mechanisms. These rheological laws yield an Arrhenius-type dependence on the temperature which has been demonstrated to control the effective viscosity of rock at large scale (Kameyama et al., 1997; Kaus \& Podladchikov, 2006; Leloup et al., 1999; Schubert \& Yuen, 1978). Field studies on ductilely deformed rocks widely support the characteristic grain size as a crucial parameter controlling effective viscosity, especially at scales at which the thermal diffusion would prevent significant viscous drop due to temperature. Indeed, natural shear zones or shear bands from the scale of a few centimeters to regional scale commonly exhibit very high and localized grain-size reduction (Carreras, 2001; Fossen \& Cavalcante, 2017; Pennachioni \& Mancktelow, 2007). This characteristic defines the mylonitic fabric. The dependence of grain size on the driving stress promoted the use of grain-size measurements as piezometers in natural mylonites (Stipp \& Tullis, 2003; Twiss, 1977).

Grain-size reduction usually results from dislocation creep through dynamic recrystallization (Derby, 1991; Derby \& Ashby, 1987; Drury \& Urai, 1990; Shimizu, 1998, 2008; Twiss, 1977). On the other hand, Newtonian creep is highly dependent on the grain size because of diffusion processes (Herring, 1950; Nabarro, 1967), but in the absence of a grain reduction mechanism, it results in grain growth. It has been proposed that the coupling between dynamic recrystallization and grain-size sensitive creep can explain plastic weakening at small scales (Drury et al., 1991; Poirier, 1980; Van der Wal et al., 1993). Dislocation creep allows the mean grain size to decrease deep into the diffusion creep domain thus producing stress weakening. Figure E1 green arrow (1) illustrates such an evolution toward the Post and Tullis (1999) piezometer (red line) in the case of a feldspar aggregate. However, Austin and Evans (2007) and Ricard and Bercovici (2009) proposed that the steady-state grain size depends on the rate of deformational work instead of the stress alone, regarding the steady-state grain size as a paleo-wattmeter instead of a paleo-piezometer. Furthermore, De Bresser et al. (2000) emphasized that such an interaction between dislocation and diffusion creep results in a grain-size equilibrium at the transition between the two mechanisms and prevents any significant weakening as schematized in Figure E1 green arrow (2). Therefore, explaining both the observed grain sizes and the formation of shear bands imposes a grain-size reduction mechanism occurring below the grain size insensitive/grain size sensitive transition grain size (Bercovici \& Ricard, 2012; Platt, 2015; Rutter \& Brodie, 1988).

The nature of this mechanism and its potential control factors remains an important question. The complete grain-size distribution and not only a characteristic grain size (Czaplinska et al., 2015; Herwegh et al., 2005; Karato, 1984; Rozel et al., 2011; Ter Heege et al., 2004) and the presence of secondary phases, a common characteristic of very fine grained shear zones (Herwegh et al., 2011; Hiraga et al., 2010; Linckens et al., 2015; Platt, 2015) have been proposed to play a role in the transition between grain-size insensitive (dislocation) to grain-size sensitive (diffusion) creep. Bercovici and Ricard (2012) proposed that grain splitting because of pinning by a second phase could reduce grain size in the diffusion creep regime. More recently, Precigout and Stunitz (2016) documented a possible grain nucleation mechanism triggered by grain boundary sliding in diffusion creep domain. This mechanism is likely to produce important grain-size reduction. High strain experiments of Cross and Skemer (2017) also produce well-mixed mylonitized textures.

The numerous studies on secondary phases, pinning, and phase mixing advocate for models with a more complete description of deforming aggregates, taking into account grain-size distributions, secondary phases, and the mixing state between them, that could be efficiently compared to field and experimental 
observations. A two-phase theory was proposed by Bercovici and Ricard (2012) using a continuum description of the system and involving the mean curvature of the interface as a measure of the phases mixing. This theory encompasses the effect of pinning and provides an easily scalable framework to compare numerical models of texture evolution to statistical measures on natural samples.

Our main objective is to propose a model allowing to compare numerical application with natural shear zones. The two-phase damage theory (Bercovici \& Ricard, 2012) was developed specifically for large-scale purposes such as plate tectonics generation and persistence (Bercovici \& Ricard, 2013). In this study, we adapt it by using the smaller-scale model to compute a texture-dependent viscosity that could be implemented in a geodynamical model and compared to field observations at both the textural and local/regional scales. We choose to account for the interdependence between interface density and the phases mean grain sizes in the entropy production equation. A modification of the equations for grain size and interface mean curvature evolution is proposed, in order to limit the effect of Zener pinning to grain growth. To account for the possible effect on the material viscosity of grain-size reduction mechanisms potentially active in the diffusion creep domain (Bercovici \& Ricard, 2012; Menegon et al., 2015; Precigout \& Stunitz, 2016; Tasaka et al., 2017), we introduce another generic deformation partition coefficient. Furthermore, we propose an equation for the effective viscosity of the material that not only depend on the effective viscosity of the two phase but also on their mixing state. For two phases $\mathrm{A}$ and $\mathrm{B}$, this mixing state is measured from the relative quantity of $\mathrm{AB}$ and $\mathrm{AA}, \mathrm{BB}$ grain-boundaries densities (Heilbronner \& Barrett, 2014). Eventually, we modify the scale numbers proposed in Bercovici and Ricard (2012) in order to account for different mineral rheologies. The variables used are specifically designed to be measurable on natural samples in order to provide useful constraints from both natural and experimental data. We finally present simple numerical applications, to display and discuss the general behavior of the equations.

\section{Continuum Description of Grained Rocks}

\subsection{Grains Properties and Size Distributions}

Following the formalism described in Bercovici and Ricard (2012, appendix A), within a control volume $\delta V$, all the macroscopic variables and their derivatives are considered continuous. In the following description, all variables accented as $\breve{X}$ are related to one grain of specific size $\breve{\mathrm{R}}$, variables accented $\tilde{X}$ are related to interfaces, and variables indexed $X_{i}$ are phase dependent $(i=1$ or2, see Appendix E and Table E3). We consider two immiscible phases represented by a function $\varphi_{i}$ such that $\varphi_{i}=1$ where only the phase $i$ is present and 0 where not. Therefore, the volume of phase $i$ in $\delta V$ is

$$
\delta V_{i}=\int_{\delta V} \varphi_{i} \mathrm{~d} V=\phi_{i} \delta V
$$

and $\phi_{i}$ satisfy $\sum_{i=1}^{2} \phi_{i}=1$. Any variables summed over the two phases is written $\bar{X}=\sum_{i} \phi_{i} X_{i}$. The volume and surface area of one grain of phase $i$ are written as the following:

$$
\begin{gathered}
\breve{V}_{i}=\frac{4}{3} \pi \breve{\mathrm{R}}_{i}^{3}, \\
\breve{\mathcal{A}}_{i}=\breve{\mathrm{A}}_{i} \breve{\mathrm{P}}_{i}=4 \pi \breve{\mathrm{R}}_{i}^{2} \breve{\mathrm{P}}_{i},
\end{gathered}
$$

which defines the grain-size variable $\breve{\mathrm{R}}_{i}$ and the grain "roughness" or "shape factor" $\breve{\mathrm{P}}_{i}$. The grain-size variable $\breve{\mathrm{R}}_{i}$ corresponds to the radius of the sphere with a volume equivalent to the grain as schematized by Figure E3a. The grain roughness $\breve{\mathrm{P}}_{i}$ accounts for the amount of area in excess from the minimum area compatible with the grain volume (the area of a sphere of radius $\breve{\mathrm{R}}_{i}$ ). All grains of a size $\breve{\mathrm{R}}_{i}$ share an identical roughness $\breve{\mathrm{P}}_{i}$. This is also illustrated in Figure E3a. Therefore, the grain volume and associated quantities only involve the variable $\breve{\mathrm{R}}_{i}$, while grain surface depends on both $\breve{\mathrm{R}}_{i}$ and $\breve{\mathrm{P}}_{i}$. The mean curvature of one grain reads

$$
\breve{\mathrm{K}}_{i}=\frac{\mathrm{d} \breve{\mathcal{A}}_{i}}{\mathrm{~d} \breve{V}_{i}}=\frac{2 \breve{P}_{i}}{\breve{R}_{i}} .
$$

Each phase within a control volume is described by a continuous and convergent distribution $v_{i}$ of grain size $\breve{\mathrm{R}}_{i}$ (the distribution function $v_{i}$ vanishes at finite maximum and minimum $\breve{\mathrm{R}}_{i}$ ). The variable $\mathcal{R}_{i}$ is the mean 
grain size and the variable $\mathcal{P}_{i}$ the mean grain roughness of the phase $i$ grain population (Appendix B). The volume of phase $i$ reads (see; Bercovici \& Ricard, 2012, appendix B, equation B.3)

$$
\delta V_{i}=\left(\int_{0}^{\infty} \breve{V}_{i} v_{i} \mathrm{~d}, R\right) \phi_{i} \delta V
$$

which because of equation (1) yields

$$
\int_{0}^{\infty} \breve{V} v_{i} \mathrm{dR}=1,
$$

implying that there is no void of any kind between the grains. The two phases being considered incompressible, we will write all mass-dependent quantities as volume dependent. The general equations concerning grain-size distributions are displayed in Appendix B.

\subsection{Interface Density and Mean Radius of Curvature}

Accounting for the effect of phase mixing on the rheology of two mineral phases materials such as the one displayed in Figure E3b is one of our objectives. The interface area is defined as the sum of all the surfaces separating a grain of phase A from a grain of the phase B in a given volume as illustrated by the red lines in Figure E3b. On the other hand, the intraphase boundary area is defined as the sum of all the surfaces separating two grains of the same phase (green and olive green lines in Figure E3). The amount of mixing between the two phases is related to the relative quantity of interfacial area $(\mathrm{AB})$ with respect to intraphase grain boundaries AA or BB (Heilbronner \& Barrett, 2014, chapter 18). The grain boundary area defines the sum for one phase of the interface and intraphase boundary area. The total interface area $\delta A_{I}$ in the control volume $\delta V$ is written (Bercovici \& Ricard, 2012, appendix A, equation A.3)

$$
\delta A_{I}=\int_{\delta V} \alpha \mathrm{d} V,
$$

which defines the interface density $\alpha$ as the total interface area normalized by the considered volume. A grain-boundary density $\alpha_{i}$ is similarly defined and corresponds to the sum of interface and intraphase grain boundary area for all the phase $i$ grains:

$$
\alpha_{i}=\int_{0}^{\infty} \mathcal{A}_{i} v_{i} \mathrm{dR}
$$

The phase grain boundary area density is $\phi_{i} \alpha_{i}$, and the total grain boundary surface of phase $i$ yields

$$
\delta A_{i}=\int_{\delta V} \phi_{i} \alpha_{i} \mathrm{~d} V
$$

The interface density should be a function of the phase volume fraction $\phi_{i}$, the phase mean grain sizes $\mathcal{R}_{i}$, and mean roughness $\mathcal{P}_{i}$. Furthermore, $\alpha$ must vanish if either $\phi_{1}$ or $\phi_{2}=0$ which advocate for $\alpha=f\left(\phi_{1} \phi_{2}\right)$ (Bercovici et al., 2001; Bercovici \& Ricard, 2012). If the interface density has to be a function of the phases proportions and grain-size distributions, another independent variable is required in order to describe the mixing state of the phase. On the basis of the work done by Ganesan and Poirier (1990), Bercovici et al. (2001), and Bercovici and Ricard (2005), Bercovici and Ricard (2012) propose the general formula:

$$
\alpha=3 \phi_{1} \phi_{2} \mathcal{F}=\frac{3 \phi_{1} \phi_{2}}{r}
$$

thus introducing the interface mean radius of curvature $r$ as an independent variable measuring the interface coarseness (inverse of interface fineness $\mathcal{F}$ ). However, in the following development, we will consider the interface density as a function of not only phases volumic fractions $\phi_{i}$ and interface mean radius of curvature $r$ but also of the mean grain sizes $\mathcal{R}_{i}$, that is, $\alpha=f\left(\phi_{i}, r, \mathcal{R}_{i}\right)$.

\section{Thermodynamics of Coupled Two-Phase Deformation}

\subsection{Damage, Self-Similarity, and Entropy Production}

Grain "damage" is related to the processes affecting grains evolution during deformation (Ricard \& Bercovici, 2009). The damage theory is generally based on the proposition that the internal energy $E$ of a 
given system depends on its entropy $S$ and density $\rho$ but also on a third state variable $\underline{D}$ related to damage, that is, $E=f(S, \rho, \underline{D})$. However, if $\rho$ and $S$ are clearly conjugated to the pressure $P$ and the temperature $T$, the conjugate variable of $\underline{D}$ is only defined by the partial derivative of the internal energy with respect to $\underline{D}$. The approach proposed by Bercovici et al. (2001) considers the damage variable $\mathcal{D}$ as the quantity of interfacial surface energy present in the system. Thus, the internal energy is now written as $E=f(S, \rho, \alpha)$ where $\alpha$ is the interfacial area density. The conjugate variable of $\alpha$ becomes $\tilde{\gamma}_{I}$ the interfacial surface tension. Therefore, grain damage results in storage of deformation energy as surface tension energy through the creation of grain boundary area. Even if mylonitized rocks exhibit a strong anisotropic fabric, we follow Bercovici and Ricard (2012) and assume a scalar $\alpha$ (grains properties are isotropic). As they demonstrated, mass conservation implies to split the grain-size fluxes in the grain-size space into continuous (grains vanishing or growing) and discontinuous fluxes (grains breaking or fusing, see Appendix A for further details).

Furthermore, Rozel et al. (2011) consider that the grain-size distribution remains self-similar throughout deformation (all distribution moments are only function of the mean). The self-similar grain-size distribution function $v_{i}$ reads

$$
v_{i}=B\left(\mathcal{R}_{i}\right) H(u)
$$

The function $B$ is an amplitude depending only on $\mathcal{R}_{i}, H$ is the shape function of the distribution which depends on the self-similarity variable $u=\breve{\mathrm{R}} / \mathcal{R}_{i}$. Rozel et al. (2011) comes up with an equation for grain-size evolution where the continuous fluxes of grain size are related to grain coarsening and the discontinuous fluxes corresponds to grain damage:

$$
\frac{\mathrm{d} \mathcal{R}_{i}}{\mathrm{~d} t}=C\left(\mathcal{R}_{i}\right)-D\left(\mathcal{R}_{i}\right)
$$

where $C\left(\mathcal{R}_{i}\right)$ and $D\left(\mathcal{R}_{i}\right)$, respectively, represent the continuous and discontinuous fluxes of mean grain sizes $\mathcal{R}_{i}$ (see section B2 and equation (B11)). The two phases grain-size distributions are assumed to be log-normal (Rozel et al., 2011), which allows to compute the various integrands related to grain-size distribution. From these leading hypothesis, it becomes possible to write the entropy production within a control volume under deformation (section C2) and to use it in order to derive phenomenological evolution laws for mean grain sizes and interface density. The main difference with the previous model at this stage is that we consider the interface density alpha to depend not only on phase volumic fractions $\phi_{i}$ and interface mean curvature $r$ but also on the phases mean grain size $\mathcal{R}_{i}$. As a consequence, we cannot assume the mean grain size and interface density to satisfy entropy positivity independently. However, using Onsager's phenomenological relations requires each thermodynamical flux to be independent from the other. We therefore rewrite the interface density $\alpha$ as the sum of all its partial derivative with respect to the grain size $\mathcal{R}_{i}$, the phase volumic fraction $\phi_{i}$, and the interface mean radius of curvature $r$. Assuming that the grain-size distribution of each phase remain self-similar, assuming that these grain-size distributions are log-normal and neglecting heat production and transfer terms, because the thermal diffusion overcomes the heat produced by viscous dissipation (high Brinkmann number) at the scales of interest,

yields the equation for the production of entropy (see section C2 and equation (C20)):

$$
\Sigma=\sum_{i}\left[\frac{3 \lambda_{2} \phi_{i} \gamma_{i} \mathcal{P}_{i}}{\lambda_{3} \mathcal{R}_{i}^{2}}-\gamma_{I} \frac{\partial \alpha}{\partial \mathcal{R}_{i}}\right] \frac{\mathrm{d} \mathcal{R}_{i}}{\mathrm{~d} t}-\gamma_{I} \frac{\partial \alpha}{\partial r} \frac{\partial r}{\partial t}-\left(\gamma_{I} \frac{\partial \alpha}{\partial \phi_{1}}+\Delta \Pi\right) \frac{\tilde{D} \phi_{1}}{\tilde{D} t}+\psi^{*} \geqslant 0
$$

The first term corresponds to the processes related to grain-size evolution and encompasses the interface variation due to grain size $\left(-\gamma_{I} \partial \alpha / \partial \mathcal{R}_{i}\right.$ term). The second term corresponds to processes that modify interface density independently of grain size (for instance, phase mixing at constant mean grain sizes). The third term is relative to phase volumic fraction, $\Delta \Pi$ being the effective pressure difference defined as $\Delta \Pi=\Pi_{2}-\Pi_{1}$ and $\Pi_{i}=P_{i}+\gamma_{i} \mathcal{K}_{i}$ (see sections $\mathrm{C} 1$ and $\mathrm{C} 2$, the mean grain curvature $\mathcal{K}_{i}$ is defined by equation (4)). The intraphase boundary surface tension energy is $\gamma_{i}$, while the true interfacial energy is $\tilde{\gamma}_{i}$. However, in the following development, the surface tension energy related to interface area corresponds to an effective surface tension energy $\gamma_{I}=\tilde{\gamma_{I}}-\sum_{i} \gamma_{i}$ (Bercovici \& Ricard, 2012, appendix E.1, equation E.1). The log-normal shape of the grain size allows to compute the grain-size distribution $n$th moment (section B2):

$$
\lambda_{n}=\int_{0}^{\infty} u^{n} H(u) \mathrm{d} u=e^{n^{2} \sigma^{2} / 2}
$$


where again $u$ is the self-similarity variable (see equation (11)), and $\sigma$ is the dimensionless variance. An important quantity is the grain boundaries density $\alpha_{i}$ that is computed from the equations (8) and (11) for self-similar log-normal distribution (see section B1 and equation (B6) for the form of $v_{i}$ ):

$$
\alpha_{i}=\int_{0}^{\infty} \mathcal{A}_{i} v_{i} \mathrm{dR}=\frac{3 \lambda_{2} \mathcal{P}_{i}}{\lambda_{3} \mathcal{R}_{i}}
$$

Similarly, from equation (4), the mean grain boundaries curvature $\mathcal{K}_{i}$ reads

$$
\mathcal{K}_{i}=\int_{0}^{\infty} \breve{\mathrm{K}}_{i} v_{i} \mathrm{dR}=\frac{2 \lambda_{2} \mathcal{P}_{i}}{\lambda_{3} \mathcal{R}_{i}} .
$$

\subsection{Nonequilibrium Thermodynamics}

In order to use the equation (13) to derive evolution equations for $\mathcal{R}_{i}$ and $r$, we consider that there are only linear and homogeneous (phenomenological) kinetic relations between thermodynamical fluxes $\mathbf{J}$ and dissipative forces $\mathbf{X}$. Under this hypothesis, the entropy production equation is written as a scalar product of thermodynamical fluxes and dissipation forces yielding:

$$
\Sigma=\sum_{k=1}^{4} J_{k} X_{k}+\psi^{*} \geqslant 0 .
$$

According to Onsager (1931), satisfying the positivity of entropy production requires to write the thermodynamical fluxes $\mathbf{J}$ as

$$
J_{k}=\sum_{l} K_{k l} X_{l},
$$

where $\underline{\mathbf{K}}$ is a positive definite square matrix. Therefore, the entropy production reads

$$
\Sigma=\sum_{k, l} X_{k} K_{k l} X_{l} \geqslant 0
$$

The positive definiteness of $\underline{\mathbf{K}}$ is sufficient to ensure the positiveness of $\Sigma$. The Onsager's reciprocal relations also imply that $\underline{\mathbf{K}}$ is symmetrical. The thermodynamical fluxes are generally defined as the whole material derivative $\dot{\mathbf{Y}}$ of the state variables $\mathbf{Y}$ (Fischer et al., 2014), which reads

$$
\mathbf{Y}=\left(\begin{array}{l}
\mathcal{R}_{1} \\
\mathcal{R}_{2} \\
r \\
\phi_{1}
\end{array}\right) \quad \dot{\mathbf{Y}}=\left(\begin{array}{l}
\dot{\mathcal{R}}_{1} \\
\dot{\mathcal{R}}_{2} \\
\dot{r} \\
\dot{\phi}_{1}
\end{array}\right) .
$$

The effective deformational work rate, $\psi^{*}$, is defined as the tensorial product of the strain-rate tensor $\underline{\dot{e}}$ with the effective stress tensor $\underline{\tau}^{*}$, that is,

$$
\psi^{*}=\underline{\tau}^{*}: \underline{\dot{e}}
$$

(see section 3.5.3 and equation (122)). It is generally considered that the strain-rate tensor corresponds to the thermodynamical flux associated to a dissipative force that is the stress tensor (De Groot \& Mazur, 1984). When writing the Onsager's matrix, this results in a rheological equation relating the strain-rate tensor to a linear function of all the dissipative forces including the stress tensor. However, in the case of deforming mineral aggregates, the rheologies are nonlinear (see sections 3.5.3 and D2). Furthermore, assuming the strain rate as the thermodynamical flux induces two other questions. First, fluxes and forces that are not of the same tensorial order should be decoupled (De Groot \& Mazur, 1984). As the considered medium is assumed isotropic (mean grain sizes are scalars), this implies that the mean grain-sizes evolution equations should be decoupled from stress. Second, Austin and Evans (2007) and Ricard and Bercovici (2009) show that mean grain sizes depend on deformational work rate rather than stress alone. Keeping with this result and the phenomenological hypothesis implies that the dissipative force in that case has to be the deformational work which in turn implies the conjugated thermodynamical flux to be 1 so that the thermodynamical fluxes are written as linear functions of the deformational work rate. We acknowledge the exotic character of this choice but will demonstrate that this assumption allows to recover the general forms of the 
equations proposed by Bercovici and Ricard (2012) and Rozel et al. (2011). Therefore, we choose to define the thermodynamical fluxes $\mathbf{J}$ and dissipative forces $\mathbf{X}$ as follows:

$$
\begin{aligned}
& J_{k}=\dot{Y}_{k} \text { for } k=1, \ldots, 4, \\
& J_{5}=1, \\
& X_{k}=f_{k} \text { for } k=1, \ldots, 4, \\
& X_{5}=\psi^{*} .
\end{aligned}
$$

Taking into account equations (13) and (26), the forces are thus given by

$$
\mathbf{f}=\left(\begin{array}{c}
\left(\frac{3 \lambda_{2} \phi_{1} \gamma_{1} p_{1}}{\lambda_{3} \mathcal{R}_{1}^{2}}-\gamma_{I} \frac{\partial \alpha}{\partial \mathcal{R}_{1}}\right) \\
\left(\frac{3 \lambda_{2} \phi_{2} \gamma_{2} p_{2}}{\lambda_{3} \mathcal{R}_{2}^{2}}-\gamma_{I} \frac{\partial \alpha}{\partial \mathcal{R}_{2}}\right) \\
-\gamma_{I} \frac{\partial \alpha}{\partial r} \\
-\left(\gamma_{I} \frac{\partial \alpha}{\partial \phi_{1}}+\Delta \Pi\right) \\
\psi^{*}
\end{array}\right)
$$

This yields the entropy production equation:

$$
\Sigma(\dot{\mathbf{Y}}, \mathbf{Y})=\sum_{k=1}^{4} f_{k} \dot{Y}_{k}+\psi^{*} \geqslant 0 .
$$

The Onsager's reciprocal relations imply $K_{k l}=K_{l k}$, yielding the corresponding system of phenomenological relations:

$$
\begin{aligned}
& \dot{\mathcal{R}}_{1}=K_{11} f_{1}+K_{12} f_{2}+K_{13} f_{3}+K_{14} f_{4}+K_{15} \psi^{*}, \\
& \dot{\mathcal{R}}_{2}=K_{12} f_{1}+K_{22} f_{2}+K_{23} f_{3}+K_{24} f_{4}+K_{25} \psi^{*}, \\
& \dot{r}=K_{13} f_{1}+K_{23} f_{2}+K_{33} f_{3}+K_{34} f_{4}+K_{35} \psi^{*}, \\
& \dot{\phi}_{1}=K_{14} f_{1}+K_{24} f_{2}+K_{34} f_{3}+K_{44} f_{4}+K_{45} \psi^{*}, \\
& 1=K_{15} f_{1}+K_{25} f_{2}+K_{35} f_{3}+K_{45} f_{4}+K_{55} \psi^{*},
\end{aligned}
$$

where we should have $K_{k k} \geqslant 0$ and $K_{k k} K_{l l} \geqslant \frac{1}{4}\left(K_{k l}+K_{l k}\right)^{2}$ for $\underline{\mathbf{K}}$ to be positive definite.

\subsection{Phases Proportions}

As we neither consider phase transition nor any mass transfer between the two phases, and we assume equivalent velocities between the two phases (see section 3.2), we prescribe $\tilde{D} \phi_{i} / \tilde{D} t=0$ in a given control volume. Furthermore, we consider all the equations (28)-(30), and (32) to be decoupled from $\dot{\phi}_{1}(31)$ which imposes $K_{k 4}=0$. The phases volumic fractions $\phi_{i}$ will be considered as constant parameters. The system thus reduces to

$$
\begin{aligned}
& \dot{\mathcal{R}}_{1}=K_{11} f_{1}+K_{12} f_{2}+K_{13} f_{3}+K_{15} \psi^{*}, \\
& \dot{\mathcal{R}}_{2}=K_{12} f_{1}+K_{22} f_{2}+K_{23} f_{3}+K_{25} \psi^{*}, \\
& \dot{r}=K_{13} f_{1}+K_{23} f_{2}+K_{33} f_{3}+K_{35} \psi^{*}, \\
& \dot{\phi}_{1}=0, \\
& 1=K_{15} f_{1}+K_{25} f_{2}+K_{35} f_{3}+K_{55} \psi^{*} .
\end{aligned}
$$


To achieve the $\dot{\phi}_{1}=0$ condition, either $K_{44}$ or $f_{4}$ or both has to be 0 . The condition

$$
f_{4}=0,
$$

taking into account the expression of the dissipation force $\mathfrak{f}_{4}$ (equation (26)) yields

$$
-\gamma_{I} \frac{\partial \alpha}{\partial \phi_{1}}=\Delta \Pi
$$

According to Bercovici and Ricard (2012), the pressure difference term reads $\Delta \Pi=\Pi_{2}-\Pi_{1}$ where $\Pi_{i}$ is an effective pressure taking into account the effect of surface tension, that is, $\Pi_{i}=P_{i}+\gamma_{i} \mathcal{K}_{i}$ (see Appendix C). Equation (39) describes the equilibrium between the variation of surface tension on the interface and the pressure difference on both side of the interface. It is equivalent to Bercovici and Ricard (2012, appendix E, equation E.23a) and corresponds to the Laplace quasi-static equilibrium across the interface.

\subsection{Grain-Size Evolution Equation Without Deformation}

The system now reads

$$
\begin{gathered}
\dot{\mathcal{R}}_{1}=K_{11} f_{1}+K_{12} f_{2}+K_{13} f_{3}+K_{15} \psi^{*}, \\
\dot{\mathcal{R}}_{2}=K_{12} f_{1}+K_{22} f_{2}+K_{23} f_{3}+K_{25} \psi^{*}, \\
\dot{r}=K_{13} f_{1}+K_{23} f_{2}+K_{33} f_{3}+K_{35} \psi^{*}, \\
1=K_{15} f_{1}+K_{25} f_{2}+K_{35} f_{3}+K_{55} \psi^{*} .
\end{gathered}
$$

At this point, we assume that the diffusion processes occurring in each phase are independent, the phases are only coupled through the evolution of interface density, this assumption implies $K_{12}=0$, and the system comes down to

$$
\begin{aligned}
& \dot{\mathcal{R}}_{1}=K_{11} f_{1}+K_{13} f_{3}+K_{15} \psi^{*}, \\
& \dot{\mathcal{R}}_{2}=K_{22} f_{2}+K_{23} f_{3}+K_{25} \psi^{*}, \\
& \dot{r}=K_{13} f_{1}+K_{23} f_{2}+K_{33} f_{3}+K_{35} \psi^{*}, \\
& 1=K_{15} f_{1}+K_{25} f_{2}+K_{35} f_{5}+K_{55} \psi^{*} .
\end{aligned}
$$

\subsubsection{Mono-Phase Aggregate}

For one phase $\left(\phi_{1}=1\right)$, considering the continuous and discontinuous grain-size fluxes to independently satisfy the positivity of entropy (Ricard \& Bercovici, 2009), and if there is no deformation, we are left with

$$
\dot{\mathcal{R}}_{1}=K_{11} f_{1}+K_{13} f_{3},
$$

which, once introduced the expression of the forces (equation (26)) and taking into account that $\alpha$ must vanish if either $\phi_{1}$ or $\phi_{2}=0$, yields

$$
\dot{\mathcal{R}}_{1}=\frac{3 \lambda_{2} \phi_{1} \gamma_{1} \mathcal{P}_{1}}{\lambda_{3} \mathcal{R}_{1}^{2}} K_{11}
$$

This equation appears similar to the equation (12) proposed by Rozel et al. (2011) in the case of no deformation $\left(\mathcal{D}\left(\mathcal{R}_{i}\right)=0\right)$ :

$$
\dot{\mathcal{R}}_{i}=C\left(\mathcal{R}_{i}\right) .
$$

Following Ricard and Bercovici (2009) and Rozel et al. (2011), we consider that, in the absence of deformation, the grain size evolution results from mass diffusive transfer driven by surface tension minimization, 
that is, classical grain growth. This implies that the coefficient $K_{11}$ should be related to the rate of growth of the grains volume and yields

$$
\begin{aligned}
\dot{\mathcal{R}}_{1} & =C\left(\mathcal{R}_{1}\right) \\
& =\frac{3 \lambda_{2} \phi_{1} \gamma_{1} \mathcal{P}_{1}}{\lambda_{3} \mathcal{R}_{1}^{2}} K_{11} \\
& =\frac{G_{1}}{p \mathcal{R}_{1}^{p-1}},
\end{aligned}
$$

where $G_{1}$ is the rate $\left([\mathrm{m}]^{2} .[\mathrm{s}]^{-1}\right)$ at which the mean grain size grows in order to decrease surface tension and $p$ is the grain-growth power exponent (Bercovici \& Ricard, 2012; Rozel et al., 2011). Taking all these remarks into account yields a $K_{11}$ coefficient of the form:

$$
K_{11}=\frac{\lambda_{3} G_{1} \mathcal{R}_{1}}{6 \phi_{1} \lambda_{2} \mathcal{P}_{1}} .
$$

\subsubsection{Two-Phase Aggregate}

\subsubsection{Grain-Size Evolution Equation}

The discontinuous fluxes which depend on deformational work rate are related to grain damage (Ricard \& Bercovici, 2009; Rozel et al., 2011). In the absence of deformation, the deformation work terms in the system vanish, which, using the equation 52 and the expressions for the thermodynamical forces of the equation (26), yields the system:

$$
\begin{aligned}
& \dot{\mathcal{R}}_{1}=\left(\frac{3 \lambda_{2} \phi_{1} \gamma_{1} \mathcal{P}_{1}}{\lambda_{3} \mathcal{R}_{1}^{2}}-\gamma_{I} \frac{\partial \alpha}{\partial \mathcal{R}_{1}}\right) K_{11}-\gamma_{I} \frac{\partial \alpha}{\partial r} K_{13} \\
& \dot{\mathcal{R}}_{2}=\left(\frac{3 \lambda_{2} \phi_{2} \gamma_{2} \mathcal{P}_{2}}{\lambda_{3} \mathcal{R}_{2}^{2}}-\gamma_{I} \frac{\partial \alpha}{\partial \mathcal{R}_{2}}\right) K_{21}-\gamma_{I} \frac{\partial \alpha}{\partial r} K_{23} \\
& \dot{r}=\left(\frac{3 \lambda_{2} \phi_{1} \gamma_{1} \mathcal{P}_{1}}{\lambda_{3} \mathcal{R}_{1}^{2}}-\gamma_{I} \frac{\partial \alpha}{\partial \mathcal{R}_{1}}\right) K_{13}+\left(\frac{3 \lambda_{2} \phi_{2} \gamma_{2} \mathcal{P}_{2}}{\lambda_{3} \mathcal{R}_{2}^{2}}-\gamma_{I} \frac{\partial \alpha}{\partial \mathcal{R}_{2}}\right) K_{23}-\gamma_{I} \frac{\partial \alpha}{\partial r} K_{33} .
\end{aligned}
$$

During grain growth, the presence of interfacial area related to the second phase particles is likely to impede the migration of grain boundaries allowing the mean grain size to reach a steady-state at smaller size. This mechanism called Zener pinning has been described by Smith (1948) and widely investigated for metallurgical purposes as it constrains the influence of impurities on the rheology of metallic alloys (see; Roberts, 2008; Smith, 1948, for instance). It occurs when a migrating grain boundary passes through a smaller inclusion which increases the interface curvature, thus increasing surface tension energy and impeding grain growth (Herwegh et al., 2005; Mehl \& Hirth, 2008). In geological materials, this process extends to the grain effect of one phase on the other during grain growth. In classical grain-growth theories, the Zener pinning effect involves the second phases particles that are very small in size and proportions with respect to the major phase in such a way that they do not evolve during the coarsening of the opposite phase. In this case Hillert (1965) proposes that the pinning effect could be represented by an equation of the form:

$$
\frac{\mathrm{d} \mathcal{R}^{2}}{\mathrm{~d} t}=G\left[1-z\left(\frac{\mathcal{R}}{\mathcal{R}_{c r}}\right)^{2}\right],
$$

thus allowing a steady-state when the major phase grain-size $\mathcal{R}$ reach a given critical size $\mathcal{R}_{c r}$. Additionally, Bercovici and Ricard (2012) build a micromechanical model for Zener pinning allowing the two phases to be of relative equivalent proportions and grain sizes and end up with a comparable equation:

$$
\begin{aligned}
\frac{\mathrm{d} \mathcal{R}_{i}}{\mathrm{~d} t} & =\frac{G_{i}}{p \mathcal{R}_{i}^{p-1}}\left[1-z\left(1-\phi_{i}\right)\left(\frac{\mathcal{R}_{i}}{r}\right)^{2}\right] \\
& =\frac{G_{i}}{p \mathcal{R}_{i}^{p-1}} \mathcal{Z}_{i},
\end{aligned}
$$


where the critical grain size $\mathcal{R}_{c r}$ corresponds to the interface mean curvature radius $r$. The coefficient $z$ reads

$$
z=\frac{3 b_{\mathfrak{n}} \lambda_{\mathfrak{n}+1}}{2(\mathfrak{n}+3) \lambda_{2}},
$$

where $\mathfrak{n}$ depends on the assumed function accounting for the evolution of grain surface curvature during pinning (Bercovici \& Ricard, 2012, appendix F.4, equation F.27). We assume that each thermodynamic flux satisfies the positivity of entropy independently. The coefficients $K_{13}$ and $K_{23}$ which couple the grain size $\mathcal{R}_{i}$ and the mean radius of curvature $r$ evolution equations are thus imposed to be $0\left(K_{13}=K_{23}=0\right)$. Substituting equation (52) in (53) and (54) and factorizing reads

$$
\begin{aligned}
\dot{\mathcal{R}}_{1} & =\frac{G_{1}}{p \mathcal{R}_{1}^{p-1}}\left(1-\frac{\gamma_{I} \lambda_{3} \mathcal{R}_{1}^{2}}{3 \lambda_{2} \phi_{1} \gamma_{1} \mathcal{P}_{1}} \frac{\partial \alpha}{\partial \mathcal{R}_{1}}\right), \\
\dot{\mathcal{R}}_{2} & =\frac{G_{2}}{p \mathcal{R}_{2}^{p-1}}\left(1-\frac{\gamma_{I} \lambda_{3} \mathcal{R}_{2}^{2}}{3 \lambda_{2} \phi_{2} \gamma_{2} \mathcal{P}_{2}} \frac{\partial \alpha}{\partial \mathcal{R}_{2}}\right), \\
\dot{r} & =-\gamma_{I} \frac{\partial \alpha}{\partial r} K_{33} .
\end{aligned}
$$

In the case of grain growth alone, we aim to recover the form of the Zener pinning coefficient proposed by Bercovici and Ricard (2012). Comparing equations 59 and (60) with (57) yields

$$
\begin{aligned}
& 1-z\left(1-\phi_{1}\right)\left(\frac{\mathcal{R}_{1}}{r}\right)^{2}=1-\frac{\gamma_{I} \lambda_{3} \mathcal{R}_{1}^{2}}{3 \lambda_{2} \phi_{1} \gamma_{1} \mathcal{P}_{1}} \frac{\partial \alpha}{\partial \mathcal{R}_{1}}, \\
& 1-z\left(1-\phi_{2}\right)\left(\frac{\mathcal{R}_{2}}{r}\right)^{2}=1-\frac{\gamma_{I} \lambda_{3} \mathcal{R}_{2}^{2}}{3 \lambda_{2} \phi_{2} \gamma_{2} \mathcal{P}_{2}} \frac{\partial \alpha}{\partial \mathcal{R}_{2}},
\end{aligned}
$$

and thus allows to constrain the partial derivatives of the interface density:

$$
\begin{aligned}
\frac{\partial \alpha}{\partial \mathcal{R}_{1}} & =\frac{3 \lambda_{2} \phi_{1} \phi_{2} z \gamma_{1} \mathcal{P}_{1}}{\lambda_{3} \gamma_{I} r^{2}}, \\
\frac{\partial \alpha}{\partial \mathcal{R}_{2}} & =\frac{3 \lambda_{2} \phi_{1} \phi_{2} z \gamma_{2} \mathcal{P}_{2}}{\lambda_{3} \gamma_{I} r^{2}} .
\end{aligned}
$$

The simplest solution of this system is

$$
\alpha=\frac{3 \lambda_{2} \phi_{1} \phi_{2} z}{\lambda_{3} \gamma_{I} r^{2}}\left(\sum_{i} \gamma_{i} \mathcal{P}_{i} \mathcal{R}_{i}\right)+\sum_{i} I_{i},
$$

with $I_{i}$ being the integrand constant relative to phase $i$. This expression appears consistent with the form of the interface density $\alpha$ used by Bercovici and Ricard (2012) to derive their two-phase model (see equation (7)). In the absence of a better choice, we will further assume the two integrand constants $I_{i}$ to be zero.

3.4.2.2. Interface Mean Radius of Curvature Evolution

From deriving the equation (64) with respect to the interface mean curvature radius $r$, one can write

$$
\frac{\partial \alpha}{\partial r}=-\frac{6 \lambda_{2} \phi_{1} \phi_{2} z}{\lambda_{3} \gamma_{I} r^{3}} \sum_{i} \gamma_{i} \mathcal{P}_{i} \mathcal{R}_{i}
$$

which yields from equation (61):

$$
\dot{r}=\frac{6 \lambda_{2} \phi_{1} \phi_{2} z}{\lambda_{3} r^{2}} \sum_{i} \gamma_{i} \mathcal{P}_{i} \mathcal{R}_{i} K_{33}
$$

According to Bercovici and Ricard (2012, appendix E, equation E.23b), the coarsening term of the interface evolution equation reads

$$
\dot{r}=\frac{3 \phi_{1} \phi_{2} \hat{G}_{I}}{q r^{q-1}} .
$$


To remain consistent with this expression but keeping with the dependence of $\alpha$ on $\mathcal{R}_{i}$, we impose that

$$
K_{33}=\frac{\hat{G}_{I}}{4},
$$

which provided that the interface coarsening contributions of phases 1 and 2 written as $G_{I i} / \gamma_{i}$ are equal to the interface coarsening rate $\hat{G}_{I}$ :

$$
\frac{G_{I 1}}{\gamma_{1}}=\frac{G_{I 2}}{\gamma_{2}}=\hat{G}_{I},
$$

and that $q=4$ yields the interface coarsening expression:

$$
\dot{r}=\frac{6 \phi_{1} \phi_{2} \lambda_{2} z \hat{G}_{I}}{\lambda_{3} q r^{q-1}} \sum_{i} \mathcal{P}_{i} \mathcal{R}_{i} .
$$

3.5. Grain Growth and Damage Evolution Laws 3.5.1. Time Evolution of a Mono-Phase Aggregate With Deformation Using equation (52) and considering that $\alpha=0$ if $\phi_{1}$ or $\phi_{2}=0$ leads from equations (44) and (47) to

$$
\begin{aligned}
\dot{\mathcal{R}}_{1} & =\frac{G_{1}}{p \mathcal{R}_{1}^{p-1}}+K_{15} \psi^{*}, \\
1 & =K_{15} \frac{3 \lambda_{2} \phi_{1} \gamma_{1} \mathcal{P}_{1}}{\lambda 3 \mathcal{R}_{1}^{2}}+K_{55} \psi^{*} .
\end{aligned}
$$

The last term in equation (73) depending on $\psi^{*}$ is related to damage. It has to be negative as damage must reduce grain size (see equation (12)), but the deformation work rate $\psi^{*}$ is positive. Thus, the coefficient $K_{15}$ must be negative. Recasting the equation (74) as

$$
K_{15} \frac{3 \lambda_{2} \phi_{1} \gamma_{1} \mathcal{P}_{1}}{\lambda 3 \mathcal{R}_{1}^{2}}=1-K_{55} \psi^{*}
$$

it implies that

$$
1-K_{55} \psi^{*} \leqslant 0
$$

In order to remain consistent with the Rozel et al. (2011) formalism, this condition is satisfied provided that

$$
\left\{\begin{array}{l}
K_{55}=\frac{X}{\psi^{*}} \\
X \geqslant 1
\end{array} .\right.
$$

Furthermore, we assume that damage to grains occurs through two mechanisms:

1. Dynamic recrystallization in the dislocation creep domain; and

2. Potential grain reduction mechanisms in the diffusion creep domain (Bercovici \& Ricard, 2012; Menegon et al., 2015; Precigout \& Stunitz, 2016; Tasaka et al., 2017).

To account for these mechanisms, we propose to write the function $X$ as

$$
X=\left(1+\mathfrak{f}_{D 1}\right) F+\left(1+\mathfrak{f}_{N 1}\right)(1-F),
$$

where $f_{D 1}$ accounts for the storage of deformation energy due to dynamic recrystallization during dislocation creep, while $f_{N 1}$ corresponds to the possible grain-size reduction mechanisms active in diffusion creep. This yields the phenomenological coefficient $K_{55}$ :

$$
K_{55}=\frac{\left[\left(1+\mathfrak{f}_{D 1}\right) F+\left(1+\mathfrak{f}_{N 1}\right)(1-F)\right]}{\psi *},
$$

where

$$
\begin{aligned}
& 0 \leqslant \mathfrak{f}_{D 1} \leqslant 1, \\
& 0 \leqslant \mathfrak{f}_{N 1} \leqslant 1 .
\end{aligned}
$$


The function $F$ must constrain the activation domains of each mechanisms (Figure E1); to stay consistent with Rozel et al. (2011) equation, we set

$$
\begin{gathered}
F=\frac{\mathcal{R}_{1}^{x}}{\mathcal{R}_{c 1}^{x}+\mathcal{R}_{1}^{x}}, \\
1-F=\frac{\mathcal{R}_{c 1}^{x}}{\mathcal{R}_{c 1}^{x}+\mathcal{R}_{1}^{x}},
\end{gathered}
$$

where $\mathcal{R}_{c 1}$ is the transition grain size between the two mechanisms (field boundary grain size), defined by the equation (section D2 and equation (D13)):

$$
\mathcal{R}_{c i}=\left(\frac{b_{i}}{a_{i} \tau_{i}^{n_{i}-1}}\right)^{1 / m_{i}}
$$

and $x$ is an exponent controlling the sharpness of the transition, $F \rightarrow 1$ if $\mathcal{R}_{1} \ggg \mathcal{R}_{c 1}$ and $F \rightarrow 0$ if $\mathcal{R}_{1} \lll \mathcal{R}_{c 1}$. Substituting equations (82) and (83) into (79) yields the phenomenological coefficient:

$$
K_{55}=\frac{\left[\left(1+\mathfrak{f}_{D 1}\right) \frac{\mathcal{R}_{1}^{x}}{\mathcal{R}_{c 1}^{x}+\mathcal{R}_{1}^{x}}+\left(1+\mathfrak{f}_{N 1}\right) \frac{\mathcal{R}_{c 1}^{x}}{\mathcal{R}_{c 1}^{x}+\mathcal{R}_{1}^{x}}\right]}{\psi^{*}},
$$

which allows to constrain $K_{15}$ from equation (75):

$$
\begin{aligned}
K_{15} \frac{3 \lambda_{2} \phi_{1} \gamma_{1} \mathcal{P}_{1}}{\lambda 3 \mathcal{R}_{1}^{2}} & =\frac{\mathcal{R}_{1}^{x}}{\mathcal{R}_{c 1}^{x}+\mathcal{R}_{1}^{x}}+\frac{\mathcal{R}_{c 1}^{x}}{\mathcal{R}_{c 1}^{x}+\mathcal{R}_{1}^{x}}-\left[\left(1+\mathfrak{f}_{D 1}\right) \frac{\mathcal{R}_{1}^{x}}{\mathcal{R}_{c 1}^{x}+\mathcal{R}_{1}^{x}}+\left(1+\mathfrak{f}_{N 1}\right) \frac{\mathcal{R}_{c 1}^{x}}{\mathcal{R}_{c 1}^{x}+\mathcal{R}_{1}^{x}}\right] \\
K_{15} \frac{3 \lambda_{2} \phi_{1} \gamma_{1} \mathcal{P}_{1}}{\lambda 3 \mathcal{R}_{1}^{2}} & =-\frac{\mathfrak{f}_{D 1} \mathcal{R}_{1}^{x}}{\mathcal{R}_{c 1}^{x}+\mathcal{R}_{1}^{x}}-\frac{\mathfrak{f}_{N 1} \mathcal{R}_{c 1}^{x}}{\mathcal{R}_{c 1}^{x}+\mathcal{R}_{1}^{x}}, \\
K_{15} & =-\frac{\lambda_{3} \mathcal{R}_{1}^{2}\left(\mathfrak{f}_{D 1} \mathcal{R}_{1}^{x}+\mathfrak{f}_{N 1} \mathcal{R}_{c 1}^{x}\right)}{3 \lambda_{2} \phi_{1} \gamma_{1} \mathcal{P}_{1}\left(\mathcal{R}_{c 1}^{x}+\mathcal{R}_{1}^{x}\right)} .
\end{aligned}
$$

If we do not consider any grain-size reduction mechanism in the diffusion creep domain $\left(\mathfrak{f}_{N 1}=0\right)$ and consider spherical grains $\left(\mathcal{P}_{i}=1\right)$, we recover the grain-size evolution law proposed by Rozel et al. (2011):

$$
\dot{\mathcal{R}}_{1}=\frac{G_{1}}{p \mathcal{R}_{1}^{p-1}}-\frac{\lambda_{3} \mathcal{R}_{1}^{2} \psi^{*}}{3 \lambda_{2} \phi_{1} \gamma_{1} \mathcal{P}_{1}} F_{D 1}
$$

where $F_{D 1}$ is defined by

$$
F_{D 1}=\frac{\mathfrak{f}_{D 1} \mathcal{R}_{1}^{x}}{\left(\mathcal{R}_{c 1}^{x}+\mathcal{R}_{1}^{x}\right)}
$$

On the other hand, the existence of a nucleation mechanism yields

$$
\dot{\mathcal{R}}_{1}=\frac{G_{1}}{p \mathcal{R}_{1}^{p-1}}-\frac{\lambda_{3} \mathcal{R}_{1}^{2} \psi^{*}}{3 \lambda_{2} \phi_{1} \gamma_{1} \mathcal{P}_{1}}\left(F_{D 1}+F_{N 1}\right),
$$

with $F_{N 1}$ defined by

$$
F_{N 1}=\frac{\mathfrak{f}_{N 1} \mathcal{R}_{c 1}^{x}}{\left(\mathcal{R}_{c 1}^{x}+\mathcal{R}_{1}^{x}\right)}
$$

We are only interested here in accounting for such mechanisms and their potential roles and magnitudes in producing the textural variations observed in natural mylonites (see Figure E2). It is not in the scope of this study to propose a complete micromechanical model at grain-size scale explaining these processes, even if it could be added later by relating the partition functions $\mathfrak{f}_{D 1}$ and $\mathfrak{f}_{N 1}$ to grain-scale micromechanical models. 3.5.2. Time Evolution of a Two-Phase Aggregate With Deformation Reintroducing all the interface dependent terms in the system yields

$$
\dot{\mathcal{R}}_{1}=\frac{G_{1}}{p \mathcal{R}_{1}^{p-1}} \mathcal{Z}_{1}+K_{15} \psi^{*}
$$




$$
\begin{gathered}
\dot{\mathcal{R}}_{2}=\frac{G_{2}}{p \mathcal{R}_{2}^{p-1}} \mathcal{Z}_{2}+K_{25} \psi^{*}, \\
\dot{r}=\frac{6 \phi_{1} \phi_{2} \lambda_{2} z \hat{G}_{I}}{\lambda_{3} q r^{q-1}} \sum_{i} \mathcal{P}_{i} \mathcal{R}_{i}+K_{35} \psi^{*}, \\
1=K_{15} \frac{3 \lambda_{2} \phi_{1} \gamma_{1} \mathcal{P}_{1}}{\lambda_{3} \mathcal{R}_{1}^{2}} \mathcal{Z}_{1}+K_{25} \frac{3 \lambda_{2} \phi_{2} \gamma_{2} \mathcal{P}_{2}}{\lambda_{3} \mathcal{R}_{2}^{2}} \mathcal{Z}_{2}-K_{35} \frac{6 \lambda_{2} \phi_{1} \phi_{2} z}{\lambda_{3} r^{3}}\left(\gamma_{1} \mathcal{P}_{1} \mathcal{R}_{1}+\gamma_{2} \mathcal{P}_{2} \mathcal{R}_{2}\right)+K_{55} \psi^{*} .
\end{gathered}
$$

Again, we recast the equation (95) in the same form than in equation (75) yielding:

$$
1-K_{55} \psi^{*}=K_{15} \frac{3 \lambda_{2} \phi_{1} \gamma_{1} \mathcal{P}_{1}}{\lambda_{3} \mathcal{R}_{1}^{2}} \mathcal{Z}_{1}+K_{25} \frac{3 \lambda_{2} \phi_{2} \gamma_{2} \mathcal{P}_{2}}{\lambda_{3} \mathcal{R}_{2}^{2}} \mathcal{Z}_{2}-K_{35} \frac{6 \lambda_{2} \phi_{1} \phi_{2} Z}{\lambda_{3} r^{3}}\left(\gamma_{1} \mathcal{P}_{1} \mathcal{R}_{1}+\gamma_{2} \mathcal{P}_{2} \mathcal{R}_{2}\right)
$$

Because the coefficients $K_{i 5}$ must be negative and to remain consistent with Bercovici and Ricard (2012) formalism, we propose to write the phenomenological coefficient $K_{55}$ :

$$
K_{55}=\frac{\phi_{1}\left[\left(1+\mathfrak{f}_{D 1}\right) \mathcal{R}_{1}^{x}+\left(1+\mathfrak{f}_{N 1}\right) \mathcal{R}_{c 1}^{x}\right]}{\psi^{*}\left(\mathcal{R}_{1}^{x}+\mathcal{R}_{c 1}^{x}\right)}+\frac{\phi_{2}\left[\left(1+\mathfrak{f}_{D 2}\right) \mathcal{R}_{2}^{x}+\left(1+\mathfrak{f}_{N 2}\right) \mathcal{R}_{c 2}^{x}\right]}{\psi^{*}\left(\mathcal{R}_{2}^{x}+\mathcal{R}_{c 2}^{x}\right)},
$$

with $\mathfrak{f}_{D i}$ the deformational work partition fraction relative to dislocation creep and dynamic recrystallization processes and $\mathfrak{f}_{N i}$ a deformational work rate partition fraction accounting for grain-size reduction mechanisms occurring in the diffusion creep domain. In each case a portion of this energy is stored by creating intraphase grain boundaries, while the rest of available energy is stored through the creation of interface surface. In order to account for these energy storage processes, we introduce the forms

$$
\begin{aligned}
& \mathfrak{f}_{D i}=\mathfrak{f}_{\alpha i} \mathfrak{f}_{D i}+\left(1-\mathfrak{f}_{\alpha i}\right) \mathfrak{f}_{D i}, \\
& \mathfrak{f}_{N i}=\mathfrak{f}_{\alpha i} \mathfrak{f}_{N i}+\left(1-\mathfrak{f}_{\alpha i}\right) \mathfrak{f}_{N i} .
\end{aligned}
$$

Taking into account equation (97), the left-hand side of equation (96) becomes

$$
\begin{aligned}
1-K_{55} \psi^{*}=-\phi_{1}\left[\mathfrak{f}_{\alpha 1}\left(F_{D 1}+F_{N 1}\right)+\left(1-\mathfrak{f}_{\alpha 1}\right)\left(F_{D 1}+F_{N 1}\right)\right] \\
-\phi_{2}\left[\mathfrak{f}_{\alpha 2}\left(F_{D 2}+F_{N 2}\right)+\left(1-\mathfrak{f}_{\alpha 2}\right)\left(F_{D 2}+F_{N 2}\right)\right],
\end{aligned}
$$

where $F_{D i}$ and $F_{N i}$ are defined by equations (89) and (91). By comparing this expression with the right-hand side of equation (96), we propose

$$
\begin{aligned}
K_{15} \frac{3 \lambda_{2} \phi_{1} \gamma_{1} \mathcal{P}_{1}}{\lambda_{3} \mathcal{R}_{1}} \mathcal{Z}_{1} & =-\phi_{1} \mathfrak{f}_{\alpha 1}\left(F_{D 1}+F_{N 1}\right), \\
K_{25} \frac{3 \lambda_{2} \phi_{2} \gamma_{2} \mathcal{P}_{2}}{\lambda_{3} \mathcal{R}_{2}} \mathcal{Z}_{2} & =-\phi_{2} \mathfrak{f}_{\alpha 2}\left(F_{D 2}+F_{N 2}\right), \\
K_{35} \frac{6 \lambda_{2} \phi_{1} \phi_{2} Z}{\lambda_{3} r^{3}}\left(\gamma_{1} \mathcal{P}_{1} \mathcal{R}_{1}+\gamma_{2} \mathcal{P}_{2} \mathcal{R}_{2}\right) & =-\sum_{i} \phi_{i}\left(1-\mathfrak{f}_{\alpha i}\right)\left(F_{D i}+F_{N i}\right),
\end{aligned}
$$

which yields the following expressions for the phenomenological coefficients:

$$
\begin{gathered}
K_{15}=-\frac{\lambda_{3} \mathcal{R}_{1}^{2} \mathfrak{f}_{\alpha 1}}{3 \lambda_{2} \gamma_{1} \mathcal{P}_{1} \mathcal{Z}_{1}}\left(F_{D 1}+F_{N 1}\right), \\
K_{25}=-\frac{\lambda_{3} \mathcal{R}_{2}^{2} \mathfrak{f}_{\alpha 2}}{3 \lambda_{2} \gamma_{2} \mathcal{P}_{2} \mathcal{Z}_{2}}\left(F_{D 2}+F_{N 2}\right), \\
K_{35}=-\frac{\lambda_{3} r^{3}\left[\sum_{i} \phi_{i}\left(1-\mathfrak{f}_{\alpha i}\right)\left(F_{D i}+F_{N i}\right)\right]}{6 \lambda_{2} \phi_{1} \phi_{2} z\left(\gamma_{1} \mathcal{P}_{1} \mathcal{R}_{1}+\gamma_{2} \mathcal{P}_{2} \mathcal{R}_{2}\right)} .
\end{gathered}
$$


Replacing equations (104), (105), and (106) into, respectively, (92), (93), and (94), the set of evolution equations reads

$$
\begin{aligned}
\dot{\mathcal{R}}_{1} & =\frac{G_{1}}{p \mathcal{R}_{1}^{p-1}} \mathcal{Z}_{1}-\frac{\lambda_{3} \mathcal{R}_{1}^{2} \mathfrak{f}_{\alpha 1}}{3 \lambda_{2} \gamma_{1} \mathcal{P}_{1} \mathcal{Z}_{1}}\left(F_{D 1}+F_{N 1}\right) \psi^{*}, \\
\dot{\mathcal{R}}_{2} & =\frac{G_{2}}{p \mathcal{R}_{2}^{p-1}} \mathcal{Z}_{2}-\frac{\lambda_{3} \mathcal{R}_{2}^{2} \mathfrak{f}_{\alpha 2}}{3 \lambda_{2} \gamma_{2} \mathcal{P}_{2} \mathcal{Z}_{2}}\left(F_{D 2}+F_{N 2}\right) \psi^{*}, \\
\dot{r} & =\frac{6 \phi_{1} \phi_{2} \lambda_{2} z \hat{G}_{I}}{\lambda_{3} q r^{q-1}} \sum_{i} \mathcal{P}_{i} \mathcal{R}_{i}-\frac{\lambda_{3} r^{3} \sum_{i} \phi_{i}\left(1-\mathfrak{f}_{\alpha i}\right)\left(F_{D i}+F_{N i}\right)}{6 \lambda_{2} \phi_{1} \phi_{2} z\left(\sum_{i} \gamma_{i} \mathcal{P}_{i} \mathcal{R}_{i}\right)} \psi^{*} .
\end{aligned}
$$

We consider the Zener pinning effect to be only related to grain growth, and we therefore aim to limit the effect of Zener pinning to the grain-growth member of the grain-size evolution equation $\left(C\left(\mathcal{R}_{i}\right)\right.$ equation 12). For this purpose, a variable change is necessary in order to allow for the Zener coefficient $\mathcal{Z}_{i}$ to decrease interface curvature which is an expected effect of Zener pinning (distorting interface), while grain-size damage is the result of deformation energy storage on grain boundaries. To do so, we write $\mathfrak{f}_{\alpha i}$ as

$$
\mathfrak{f}_{\alpha i}=\chi_{i}-\chi_{i} \phi_{i} z\left(\frac{\mathcal{R}_{i}}{r}\right)^{2} .
$$

This yields

$$
1-\mathfrak{f}_{\alpha i}=1-\chi_{i}+\chi_{i} \phi_{i} z\left(\frac{\mathcal{R}_{i}}{r}\right)^{2}
$$

Eventually, substituting equation (111) into (107)-(109), the final set of evolution equations reads

$$
\begin{aligned}
& \dot{\mathcal{R}}_{1}=\frac{G_{1}}{p \mathcal{R}_{1}^{p-1}} \mathcal{Z}_{1}-\frac{\lambda_{3} \mathcal{R}_{1}^{2} \chi_{1}}{3 \lambda_{2} \gamma_{1} \mathcal{P}_{1}}\left(F_{D 1}+F_{N 1}\right) \psi^{*}, \\
& \dot{\mathcal{R}}_{2}=\frac{G_{2}}{p \mathcal{R}_{2}^{p-1}} \mathcal{Z}_{2}-\frac{\lambda_{3} \mathcal{R}_{2}^{2} \chi_{2}}{3 \lambda_{2} \gamma_{2} \mathcal{P}_{2}}\left(F_{D 2}+F_{N 2}\right) \psi^{*}, \\
& \dot{r}=\frac{6 \phi_{1} \phi_{2} \lambda_{2} z \hat{G}_{I}}{\lambda_{3} q r^{q-1}} \sum_{i} \mathcal{P}_{i} \mathcal{R}_{i}-\frac{\lambda_{3} r^{3} \sum_{i} \phi_{i}\left(1-\chi_{i} \mathcal{Z}_{i}\right)\left(F_{D i}+F_{N i}\right)}{6 \lambda_{2} \phi_{1} \phi_{2} z \sum_{i} \gamma_{i} \mathcal{P}_{i} \mathcal{R}_{i}} \psi^{*} .
\end{aligned}
$$

\subsubsection{Two-Phase Deformational Work Rate}

\subsubsection{Mixing Variables}

For two phases $\mathrm{A}$ and $\mathrm{B}$, the mixing state of the aggregate is defined by the relative quantity of interfacial area $\mathrm{AB}$ and intraphase grain boundaries areas AA and BB (Heilbronner \& Barrett, 2014, chapter 18). Normalized by the volume, these quantities are the interface density $\alpha$ and the intraphase grain boundaries densities $\alpha_{11}$ and $\alpha_{22}$ (which are different from the phases grain boundaries densities $\alpha_{1}$ and $\alpha_{2}$ ). According to Heilbronner and Barrett (2014, chapter 18), a relevant way to estimate the mixing state is to compute the ratio of the interface area over the sum of interface and both intraphase grain boundaries areas:

$$
S=\frac{\alpha}{\alpha+\alpha_{11}+\alpha_{22}}
$$

The interface density is given by the equation (66), and the phase intraphase grain boundaries densities could be estimated as the phase grain boundaries density minus the interface density. This is only an estimation because of the grains that are cut by the control volume surfaces; however, for very large number of grains, this error should be small (i.e., if $L$ the volume characteristic length is much larger than $\mathcal{R}_{i}$, which is already a necessary condition for continuous grain-size distributions). $S$ therefore reads

$$
S=\frac{\alpha}{\alpha_{1}+\alpha_{2}-\alpha}
$$


where $\alpha_{i}$ is given by equation (15). For a given phase volume fraction, the mixing state of the aggregate is therefore only dependent on the evolution of both phases mean grain size and interface mean curvature radius. Grain growth by removing primarily intraphase grain boundaries (coalescence of neighboring grains) increases the mixing of the phases. In two phase aggregates, dislocation creep which involves grains splitting either by subgrains rotation or bulging (Shimizu, 2008) creates intraphase grain boundaries and results in clusters formation (Raimbourg et al., 2008) thus decreasing the phase mixing. In the diffusion creep domain, the evolution of the mixing state must depend on the mechanism considered. However, fine-grained mylonite often exhibits well-mixed pattern that advocate for a grain reduction mechanism with important creation of interface such as nucleation (Dimanov et al., 2007; Precigout \& Stunitz, 2016).

\subsubsection{Phases Effective Viscosities}

It is now necessary to define the form of the deformational work $\psi^{*}$. Even if numerous microscopic, elementary processes are simultaneously involved in ductile rock deformation (dislocation glide and climb, diffusion through grain boundaries or interiors, and grain boundary sliding), the macroscopic deformation and associated flow laws are classically classified under two main types:

1. dislocation or grain size insensitive creep; and

2. diffusion or grain-size sensitive creep.

The effective viscosities associated with these two mechanisms reads (see section D2):

$$
\begin{aligned}
& \mu_{i}^{\mathrm{disl}}=\frac{1}{2}\left(a_{i} \tau_{i}^{n_{i}-1}\right)^{-1}, \\
& \mu_{i}^{\mathrm{diff}}=\frac{1}{2}\left(\frac{\mathcal{R}^{m_{i}}}{b_{i}}\right),
\end{aligned}
$$

with $\tau_{i}$ being the second invariant of the stress tensor in phase $i$. We consider that within a phase $i$, all grains share a stress $\breve{\tau}_{i}$ equivalent to the macroscopic stress $\tau^{*}$ (Bercovici \& Ricard, 2012; Rozel et al., 2011). Furthermore, the deformation of a grain can be due to the two mechanisms acting simultaneously, thus the total strain rate is assumed to be the sum of the strain rate related to each deformation mechanism. We therefore use a composite rheology of the form:

$$
\underline{\dot{e}}=\underline{\dot{e}}_{i}^{\mathrm{disl}}+\underline{\dot{e}}_{i}^{\mathrm{diff}}=\left(a_{i} \tau_{i}^{n_{i}-1}+\frac{\mathrm{B}_{i}}{\mathcal{R}_{i}^{m_{i}}}\right) \underline{\boldsymbol{\tau}}_{i},
$$

where the coefficient $\mathrm{B}_{i}=b_{i} \frac{\lambda_{3-m}}{\lambda_{3}}$ accounts for the effect of grain-size distribution variance on the overall rheology (Rozel et al., 2011). This relation defines the phase $i$ global viscosity:

$$
\mu_{i}=\frac{1}{2}\left(a_{i} \tau_{i}^{n_{i}-1}+\frac{\mathrm{B}_{i}}{\mathcal{R}_{i}^{m_{i}}}\right)^{-1} .
$$

\subsubsection{Effective Deformational Work Rate}

The simplest hypothesis is to assume the aggregate effective viscosity $\mu^{*}$ to be a volume average of the phases viscosities, we get $\mu^{*}=\bar{\mu}=\sum_{i} \phi_{i} \mu_{i}$ and $\psi^{*}=\bar{\psi}$ (Bercovici \& Ricard, 2012). However, the influence of phases mixing on the material rheological behavior may be taken into account in defining the effective deformational work rate. Therefore, the aggregate viscosity $\mu^{*}$ is written as a function of phases viscosities $\mu_{i}$, volume fractions $\phi_{i}$, and the variable $S$ quantifying the degree of mixing between the phases. Evaluating the rheology of heterogeneous materials requires a complex mathematical treatment (Drew \& Segel, 1971; Hill, 1965; Ravichandran \& Seetharaman, 1993). Here the effective viscosity is considered to be a function of the two pure phase end-members viscosities, whose relative contributions is a function of the aggregate "mixing pattern" depicted by the variable $S$. The aggregate is considered to evolve between two end-member cases:

1. The phase tends toward clustering (see Figures E2b and E2e), when $S \rightarrow 0$ (i.e., intraphase boundaries are dominant); and

2. The phase tends toward maximum mixing (see Figures E2c and E2f), when $S \rightarrow 1$ (i.e., interface boundaries are dominant).

If interface grain boundaries are dominant, the variable $S$ tends toward unity (the case $S=1$ corresponds to the perfect mixing state where there is no intraphase boundaries anymore akin to the chessboard pattern). In 
contrast, even if the texture includes large clusters, the presence of two phases implies a nonzero amount of interface. As a consequence, if $S$ could be low depending on the amount and size of grains clusters, it cannot reach zero. The evolution of the aggregate mixing state is likely to be controlled by the dominant deformation mechanism acting on each of the two phases. In order to compute the material effective viscosity, we consider that in case (1) the whole rheology is dominated by the clusters (or layers) of the weakest phase, while the homogeneous mixing of case (2) is likely to results in an average of the two phases viscosities. These remarks lead us to propose the following expression for the aggregate effective viscosity:

$$
\mu^{*}=S\left(\phi_{1} \mu_{1}+\phi_{2} \mu_{2}\right)+(1-S)\left(\frac{\mu_{1} \mu_{2}}{\phi_{1} \mu_{2}+\phi_{2} \mu_{1}}\right) .
$$

Thus, when the phases are clustered $(S \rightarrow 0)$, the first term is negligible, and the rheology is dominated by the weakest phase (harmonic mean). On the contrary, when the phase are well mixed $(S \rightarrow 1)$, the viscosity is approximated by the first term and results from a volume average of the two phases viscosities. This averaging hypothesis on the rheology of the general aggregate is still linear with respect to the strain rate, thus the total work rate acting on the control volume reads

$$
\begin{aligned}
\psi^{*} & =\underline{\tau}^{*}: \underline{\dot{e}} \\
& =2 \mu^{*} \dot{e}^{2} \\
& =2 \dot{e}^{2}\left[S\left(\phi_{1} \mu_{1}+\phi_{2} \mu_{2}\right)+(1-S)\left(\frac{\mu_{1} \mu_{2}}{\phi_{1} \mu_{2}+\phi_{2} \mu_{1}}\right)\right] .
\end{aligned}
$$

\section{Application of the Model in Mono and Two-Phase Cases}

\subsection{Dimensionless Equations and Solving Method}

In deriving dimensionless relations, we account for the possibility of different phases rheological laws. The phase 2 rheological parameters $a_{2}=\mathfrak{a}_{2} \exp \left(-\mathrm{E}_{2}^{\text {disl }} / R T\right), b_{2}=\mathfrak{b}_{2} \exp \left(-\mathrm{E}_{2}^{\text {diff }} / R T\right)$ are scaled using the corresponding parameters of the phase 1 , thus the imposed strain rate $\dot{e}$ and the rheological parameters of the phase $1, a_{1}=\mathfrak{a}_{1} \exp \left(-\mathrm{E}_{1}^{\text {disl }} / R T\right)$ and $b_{1}=\mathfrak{b}_{1} \exp \left(-\mathrm{E}_{1}^{\text {diff }} / R T\right)$ are our main scaling parameters:

$$
\begin{aligned}
\dot{e} & =\dot{e}_{s} \dot{e}^{\prime} \quad \text { with } \quad \dot{e}_{s}=\dot{e}, \\
t & =t_{s} t^{\prime} \quad \text { with } \quad t_{s}=\frac{1}{\dot{e}}, \\
\tau & =\tau_{s} \tau^{\prime} \quad \text { with } \quad \tau_{s}=\left(\frac{\dot{e}_{s}}{a_{1 s}}\right)^{\frac{1}{n_{1}}}, \\
\mathcal{R} & =\mathcal{R}_{s} \mathcal{R}^{\prime} \quad \text { with } \quad \mathcal{R}_{s}=\left(b_{1 s} \frac{\tau_{s}}{\dot{e}_{s}}\right)^{\frac{1}{m_{1}}}, \\
a_{1} & =a_{1 s} a_{1}^{\prime} \quad \text { with } \quad a_{1 s}=a_{1}, \\
\mathrm{~B}_{1} & =\mathrm{B}_{1 s} \mathrm{~B}_{1}^{\prime} \quad \text { with } \quad \mathrm{B}_{1 s}=\mathrm{B}_{1}, \\
a_{2}=a_{2 s} a_{2}^{\prime} & \text { with } \quad a_{2 s}=\frac{\dot{e}_{s}}{\tau_{s}^{n_{2}}}=\frac{\dot{e}_{s}^{1-\frac{n_{1}}{n_{2}}}}{a_{1}^{-\frac{n_{1}}{n_{2}}}}, \\
\mathrm{~B}_{2}=\mathrm{B}_{2 s} \mathrm{~B}_{2}^{\prime} & \text { with } \quad \mathrm{B}_{2 s}=\frac{\mathcal{R}_{s}^{m_{2}} \dot{e}_{s}}{\tau_{s}}=\mathrm{B}_{1}^{\frac{m_{2}}{m_{1}}} \dot{e}^{\frac{m_{2}\left(1-n_{1}\right)+m_{1}\left(n_{1}-1\right)}{m_{1} n_{1}}} a_{1}^{\frac{m_{2}-m_{1}}{m_{1} n_{1}}},
\end{aligned}
$$

where

$$
\mathrm{B}_{i}=\frac{\lambda_{3-m_{i}}}{\lambda_{3}} b_{i} .
$$

Using these scaling parameters, the rheological equation does not change and yields

$$
\dot{e}^{\prime}=a_{i}^{\prime} \tau_{i}^{\prime n_{i}}+\frac{\mathrm{B}_{i}^{\prime}}{\mathcal{R}_{i}^{\prime m_{i}}} \tau_{i}^{\prime} .
$$


From equations (112) and (113), using the scaling parameters described above, the grain size evolution law reads

$$
\begin{aligned}
\dot{\mathcal{R}}_{i}^{\prime} & =\frac{G_{i}}{p \mathcal{R}_{s}^{(p-1)} \dot{e}_{s}}\left[1-z\left(1-\phi_{i}\right)\left(\frac{\mathcal{R}^{\prime}}{r^{\prime}}\right)^{2}\right]-\frac{\lambda_{3}\left(1-\chi_{1}\right) \mathcal{R}_{i}^{\prime 2} \mathcal{R}_{s} \tau_{s}}{3 \lambda_{2} \gamma_{i} \mathcal{P}_{i}}\left(F_{D i}+F_{N i}\right) \psi^{\prime *}, \\
\dot{\mathcal{R}}_{i}{ }^{\prime p} & =\mathrm{C}_{i} \mathrm{Z}_{i}-\mathrm{D}_{i} \mathcal{R}_{1}^{\prime(p+1)}\left(F_{D i}+F_{N i}\right) \psi^{\prime *},
\end{aligned}
$$

where $\mathrm{C}_{i}, \mathrm{Z}_{i}$, and $\mathrm{D}_{i}$ are given by

$$
\begin{aligned}
\mathrm{C}_{i} & =\frac{G_{i}}{\mathcal{R}_{s}^{2} \dot{e}_{s}}, \\
\mathrm{Z}_{i} & =1-z\left(1-\phi_{i}\right)\left(\frac{\mathcal{R}_{i}^{\prime}}{r^{\prime}}\right)^{2}, \\
\mathrm{D}_{i} & =\frac{p \lambda_{3} \chi_{i} \mathcal{R}_{s} \tau_{s}}{3 \lambda_{2} \gamma_{i} \mathcal{P}_{i}} .
\end{aligned}
$$

The dimensionless mean radius of curvature equation reads from equation (114):

$$
\begin{aligned}
\dot{r}^{\prime} & =\frac{3 \phi_{1} \phi_{2} \lambda_{2} z \hat{G}_{I}}{\lambda_{3} q r^{\prime(q-1)} \mathcal{R}_{s}^{q} \dot{e}_{s}} \sum_{i} \mathcal{P}_{i} \mathcal{R}_{i}-\frac{q \lambda_{3} r^{\prime 3} \mathcal{R}_{s} \tau_{s} \sum_{i} \phi_{i}\left(1-\chi_{i} \mathrm{Z}_{i}\right)\left(F_{D i}+F_{N i}\right)}{6 \lambda_{2} \phi_{1} \phi_{2} z \sum_{i} \gamma_{i} \mathcal{P}_{i} \mathcal{R}_{i}} \psi^{\prime *}, \\
r^{\prime q} & =\mathrm{C}_{I} \sum_{i} \mathcal{P}_{i} \mathcal{R}_{i}-\mathrm{D}_{I} \frac{r^{\prime(q+2))} \sum_{i} \phi_{i}\left(1-\chi_{i} Z_{i}\right)\left(F_{D i}+F_{N i}\right)}{\sum_{i} \gamma_{i} \mathcal{P}_{i} \mathcal{R}_{i}} \psi^{\prime *},
\end{aligned}
$$

with the coefficients:

$$
\begin{gathered}
\mathrm{C}_{I}=\frac{3 \phi_{1} \phi_{2} \lambda_{2} z \hat{G}_{I}}{\lambda_{3} \mathcal{R}_{s}^{q} \dot{e}_{s}} \\
\mathrm{D}_{I}=\frac{q \lambda_{3} \mathcal{R}_{s} \tau_{s}}{6 \lambda_{2} \phi_{1} \phi_{2} z}
\end{gathered}
$$

To evaluate the behavior of the model, we assume velocity boundaries conditions to be $\mathbf{v}=u(y) \hat{\mathbf{x}}$ ( $\operatorname{simple}$ shear), applied to a horizontal layer infinite in $x$ and finite in $y$. The medium is considered uniform in both $x$ and $y$ directions so the volume fractions $\phi$ and $\phi_{2}=1-\phi$ remain constant and uniform throughout the calculation. Therefore, all material derivatives become only dependent on time $t$, and we have

$$
\frac{D}{D t}=\frac{\mathrm{d}}{\mathrm{d} t}
$$

In simple shear and zero dimension, the only imposed strain-rate tensor component is $\dot{e}=\frac{1}{2} \frac{\partial u}{\partial y}$. Thus, the only component of stress tensor is given by

$$
\tau_{i}=\left(\frac{\dot{e}}{a_{i}+\mathrm{B}_{i} \mathcal{R}_{i}^{-m_{i}} \tau_{i}^{1-n_{i}}}\right)^{1 / n i},
$$

which is evaluated numerically using a Picard fixed point scheme. This yields the deformational work on each phase $i$ through

$$
\psi_{i}=2 \dot{e} \tau_{i}
$$

Then, the total deformational work rate $\psi^{*}$ is computed using equation (115) which then allows to calculate the time evolution of $\mathcal{R}_{1}, \mathcal{R}_{2}$ (equation (134)), and $r$ (equation (139)) using a fourth-order Runge-Kutta explicit iterative scheme. The code was developed with using Matlab@). 


\subsection{Model Parameters}

\subsubsection{General Parameters and Rheological Laws}

The role of the grain-size distribution dispersion during two-phase material deformation could be important (Rozel et al., 2011). Accordingly, we choose to use rheological laws for dislocation and diffusion creep derived from the same starting material. We use the rheological parameters from Hirth and Kolhstedt (2003) for olivine flow laws, Rybacki and Dresen (2000) for anorthite, and Dimanov and Dresen (2005) for diopside. For grain-growth laws, we use the parameters proposed by Karato (1989) for olivine using an activation energy of $310^{5} \mathrm{Jmol}^{-1} \mathrm{~K}^{-1}$ (Bercovici \& Ricard, 2012). The growth laws used for anorthite and diopside are from Dresen et al. (1995) and Fisler et al. (1997), respectively. The experimental parameters are summarized in Table E1. A rough estimate from Hidaka gabbro thin section measurement (ratio of measured grain perimeter over the perimeter of a circle of equivalent area, 2-D measures) yields a value of 1.2 for the grain roughness $\mathcal{P}_{i}$, which we will adopt for our computations. Grain boundaries and interfaces surface tension energy differences are assumed close enough to be assumed equal to 1J (Bercovici \& Ricard, 2012; Duyster \& Stockhert, 2001). As the medium is considered incompressible, we do not account for activation volume and also neglect the possible effect of water fugacity. All the parameters are summarized in Table E1 below. The rheological laws for plagioclase, diopside, and olivine are plotted in a deformation map (stresses as a function of grain size) in Figure E5. We impose throughout the computation a constant strain rate of $10^{-12} \mathrm{~s}^{-1}$ as a reasonable order of magnitude for geological deformation processes.

\subsubsection{Zener Pinning Parameters}

The value of the pinning coefficient $z$ is computed using the value of $n=1$ which yields $b_{n}=1 / 20$ and $z=\frac{3 \lambda_{4}}{160 \lambda_{2}}$. The exponent of the mean interface curvature radius is $q=4$ as comes out of our equation (72) and was also proposed by Bercovici and Ricard (2012). To determine the interface mean curvature radius growth rate $\hat{G}_{I}$ by comparing the model to the grain growth experiments of Hiraga et al. (2010), Bercovici and Ricard (2012) proposed the form

$$
G_{I}=\frac{q G_{i}}{p 250} \tilde{r}^{(q-p)},
$$

where $G_{i}$ the phase growth rate considered identical for the two phases, $p$ is the grain growth exponent considered to be 2 (Hashim, 2016), and $\tilde{r}$ is the experimental interface roughness considered to be of the order $1 \mu \mathrm{m}$ (Bercovici \& Ricard, 2012, 2013). In the current case, in order to account for different grain-size growth rates, we propose

$$
\hat{G}_{I}=g_{I}\left(\phi_{2} G_{1}+\phi_{1} G_{2}\right),
$$

because in the case of $\left.\phi_{i}>>>\left(1-\phi_{i}\right)\right)$, the interface growth rate should be dominated by the less abundant phase. This equation imposes a linear dependence of $\hat{G}_{I}$ on the phases volumic fractions. Another possibility can be

$$
\hat{G}_{I}=g_{I}\left(\frac{G_{1} G_{2}}{\phi_{1} G_{1}+\phi_{2} G_{2}}\right)
$$

which yields a hyperbolic relationship between $\hat{G}_{I}$ and $\phi_{i}$. These equations are likely to be valid when $\phi_{i} \rightarrow 0$, but in the limit case where $\phi_{i}=0$, the whole equation for the interface mean radius of curvature $r$ vanishes, and this equation does not make sense anymore. We will choose the equation (146) as the simplest choice for the following numerical applications. The parameter $g_{I}$ is fixed to $7.910^{-2}$ because lower values yield unphysical negative value of the Zener coefficient $\mathcal{Z}_{i}$. The grain growth rate $G_{i}$ is given by

$$
G_{i}=g_{i}^{\text {grow }} \exp \left(\frac{-E_{i}^{\text {grow }}}{R T}\right),
$$

where $R$ is the gas constant, $T$ is the temperature, and $g_{i}$ and $E_{i}^{\text {grow }}$ are, respectively, a prefactor and an activation energy (Table E1). As the phases volumic fraction may have an important impact on the grain-sizes growth rates, we display on Figure E4 the grain sizes of during grain growth using the grain growth law of Dresen et al. (1995) for anorthite (phase 1) and Fisler et al. (1997) for enstatite (phase 2) computed with varying $\phi_{1}$. The mixing variable $S$ growth until the aggregate reach the maximum mixing possible, that is, all the intraphase boundaries have disappeared in the less abundant phase, all its grains are therefore dispersed within the "matrix" phase. As expected, the maximum $S$ value is reached for $\phi_{1}=\phi_{2}=0.5$ 
(Figure E4, cyan curves), while strongly different volumic fractions yield a weaker mixing (Figure E4, green and brown curves).

4.2.3. Deformational Work Partition Fractions

To constrain the value of the deformational work partition fraction $\mathfrak{f}_{D i}$, we consider the case of a pure phase aggregate, where according to De Bresser et al. (2000), we should obtain steady-state when the mean grain size reaches the field boundary. This assumption allows to compute the corresponding partition functions $\mathfrak{f}_{D i}$ using equation (134) in the case of $\phi_{1}=1$ and taking into account that $F_{D i}=\mathfrak{f}_{D i} / 2$ :

$$
\mathfrak{f}_{D i}=\frac{2 C i}{D_{i} \mathcal{R}_{c i}^{3} \psi^{*}},
$$

where $\mathcal{R}_{c i}$ is the transition grain size computed using equation (84), and the stresses are evaluated numerically using equation (119). With the rheological parameters summarized in Table E1, this equation yields the following $\mathfrak{f}_{D i}$ values for each phase.

The dimensionless variance $\sigma$ of the experimental grain-size distributions used to constrain the flow laws parameters is estimated from the Rybacki and Dresen (2000) for anorthite. The value of 0.8 yields a steady-state grain size on the field boundary for a partition fraction $\mathfrak{f}_{D i}$ of $9.844510^{-6}$ as depicted on Table E2 column 3. For olivine and diopside, the values for $\sigma$ and $\mathfrak{f}_{D i}$ are estimated by computing the steady-state grain size using equation (92) and comparing the resulting values to the experimental stress/grain-size curves displayed in Figure E5. For the grain sizes to achieve steady-state on the field boundary requires the value of $\sigma$ and $\mathfrak{f}_{D i}$ displayed in Table E2 columns 2 and 3, respectively. In the case of olivine, our estimated value lies within the range predicted by the Mulyukova and Bercovici (2017) parametrization $\left(\mathfrak{f}_{D i}=9.910^{-4}\right.$ to $1.510^{-11}$ depending on the chosen activation energy) for a temperature of $1,000 \mathrm{~K}$, taking into account the value of $\mathcal{P}_{i}$. For the following computation, we therefore use the values computed for the pure phase aggregate and displayed in Table E2 last column, while assuming that the two phases share similar normalized variance $\sigma$. This does not change the values computed for anorthite and olivine; however, the value of $\mathfrak{f}_{D i}$ for pyroxene using a dimensionless variance of 0.8 is displayed in Table E2 column 4.

One of the main parameter of the model is the partition function $\chi_{i}$ between intraphase and interface grain boundaries (between $\mathcal{R}_{i}$ and $r$ ). So far, we cannot explicitly constraint its potential range of values. However, a discussion on the expected behavior of our set of variables with respect to the texture evolution during deformation is able to provide significant insight on $\chi_{i}$.

First, it is expected that dislocation creep forms grain clusters (for instance, recrystallized tails around porphyroclasts; Passchier \& Trouw, 2006; Raimbourg et al., 2008), which could be deformed as the deformation goes on. In this case, when a new small grain forms, a large portion of its boundary remains "intraphase." In the extreme limit, one could think of a large grain segmenting in smaller ones without any change of its extern boundary. Within our model framework, this case would lead to a mean radius of curvature decreasing more slowly than the mean grain size yielding a decrease of $S$, the degree of mixing.

Second, when classical diffusion creep becomes the dominant mechanism, the mean grain size increases. This results from the migration of intraphase grain boundary in order to decrease surface tension energy. Simultaneously, grain growth involves a smoothing of the interface, but interfacial grain boundaries density would not decrease at the same rate than intraphase boundaries densities. Indeed, for one grain to be consumed by another one separated by the other phase requires mass transfer of one phase across the other one, a process slower than simple intraphase boundaries migration between two grains of the same phase. The corresponding evolution of our variables yields mean grain sizes $\mathcal{R}_{i}$ growing much faster than the mean interface curvature radius $r$. As a consequence, the aggregate degree of mixing increases because of grains clusters are consumed by the growing grains.

Eventually, if any processes reducing grain size is active in the diffusion creep domain, the formation of a new small grain involves the creation of an important amount of interfacial area. In this model, this results in a difference between the rate of decrease of the grain size and the interface mean radius of curvature, the later decreasing more rapidly. The main parameter controlling the relative evolution of $\mathcal{R}_{i}$ and $r$ rates is the function $\chi_{i}$. For anorthite compositions, a value below $\chi_{i}=0.5$ yields an interface mean radius of curvature of the interface decreasing more rapidly than the grain size, while a value of approximately $\chi_{i}=0.7$ leads to similar rates of evolutions. We have chosen the $\chi_{i}=1$ value to be consistent with this interpretation. 
4.3. Application to a Gabbroic Aggregate 4.3.1. Mono-Phase Deformation and Grain Size Reduction in the Diffusion Creep Regime Figure E6 depicts the effect of a grain-size reduction mechanism active in diffusion creep domain. In the case where no grain-size reduction is allowed within the diffusion creep regime (Figure E6, blue solid lines), the anorthite grain size stabilizes on the dislocation/diffusion transition grain size depicted by the blue-dotted line. The rate of grain-size decrease is controlled by the partition fraction $\mathfrak{f}_{D_{i}}$ adjusted in section 4.2.3 to match the field boundary hypothesis. The small weakening observed on the stress curve is due to the portion of the grain-size distribution that is already deforming in the diffusion creep domain when the mean grain-size approach the transition between the two domains. If a grain-size reduction mechanism is active in the diffusion creep domain (Figure E6, red solid lines), as expected, both the steady-state grain size and stress appear much smaller than the ones reached in the previous case, highlighting the role such a mechanism to weaken the material. The importance of this effect is related to the partition fraction $\mathfrak{f}_{N i}$. Within our model framework and given the chosen parameters, a portion function $\mathfrak{f}_{N i} \simeq 160 \mathfrak{f}_{D i}$ is required to reach the Post and Tullis (1999) piezometer. Therefore, if the mechanism involved remains compatible with a dissipation of deformation work under the form of surface tension energy, it has to be much more efficient. 4.3.2. Bi-Phase Aggregate, Dislocation Creep, and Phase Mixing Figure E7 depicts a deforming two-phase aggregate of gabbroic composition (70\% anorthite/30\% diopside) computed with the rheologies depicted in Table E1. If no energy storage in the diffusion creep regime is allowed (a), the two mean grain sizes $\mathcal{R}_{1}$ and $\mathcal{R}_{2}$ stabilize on the field boundary (dotted green and blue lines). The rates of evolution of the phases mean grain sizes and interface mean radius of curvature depend mostly on the value of the coarsening rate $\hat{G}_{I}$ (equation (146)). As expected, the interface density $\alpha$ achieves steady-state when the value of the smallest phase grain boundary density is reached. The mixing degree $S$ (magenta solid line) displays a decrease when dislocation creep is dominant. This is due to the small rate of decrease of $r$ with respect to $\mathcal{R}_{1}$ and $\mathcal{R}_{2}$. An important stress drop is synchronous of the mechanism transition due to the most abundant phase grain size (anorthite, blue lines) stabilizing below the field boundary. The minimum stress is reached at about 1 My.

If an active grain-size reduction mechanism is active in the diffusion creep domain $\left(\mathfrak{f}_{N i} \neq 0\right.$, Figure E7b), the behavior of the main variables appears much more disturbed. Both mean grain sizes show a sharp decrease at the transition between dislocation and diffusion creep and reach smaller values than presented in the case Figure E7a. The interface density appears to achieve steady-state near the smaller grain boundary density. The computed steady-state grain sizes appear compatible with the fine grain size and the mixture pattern observed in natural ultramylonites (Drury et al., 2011; Warren \& Hirth, 2006). The behavior of the mixing variable $S$ appears similar to the case Figure E7a during dislocation creep but displays a smaller increase when diffusion creep is dominant while the contrary would have been expected. The stress decrease is very sharp, and the smallest stress value are reached as soon as diffusion creep becomes active and much sooner (30,000 years) than the case without important grain-size reduction in the diffusion creep regime.

The final grain size and the difference between the two phases depend mostly on volumic fraction as illustrated by Figure E8. Especially, anorthite grains can reach a size compatible with the Post and Tullis (1999) piezometer for small volume fractions.

\section{Discussion}

\subsection{Model Objectives}

Natural shear zones or shear bands are characterized as "band-like structures of higher strain and vorticity than their surrounding” (Mancktelow, 2002). This definition appears valid at all the scales where deformation localization is observed, that is, from the thin section (millimeters) to regional scales (100 km). From a mechanical point of view, such a localization phenomenon requires stress weakening, which could results from great variety of control factors, over the involved range of scales (White et al., 1980). However, grain-size reduction appears to be a very common feature of deformation localization whatever the scale of observation. Whether it is a consequence or a cause of the related high strain is still largely debated. In the case of relatively homogeneous materials (granites, gabbro, and peridotite) where neither preexistent heterogeneities of higher scales nor external factors could be identified, the inherent rock mechanical properties have to be the crucial factors to explain rock complex rheologies especially deformation localization (Raimbourg et al., 2008; Sullivan et al., 2013). These mechanical properties are determined by the rock microstructural organization, that is, the rock texture. The rock texture depends both on its nature (the mineral phases that 
are present, their proportions, and chemical compositions) and its history (the deformation history and a resulting preexistent fabric and the thermal history that control the rate of grains recovery).

From a numerical point of view, geodynamical models are based on the known deformation mechanisms, and the related experimental data provided the hypothesis that the results obtained at high experimental strain rate $\left(10^{-5}\right.$ to $\left.10^{-7} \mathrm{~s}^{-1}\right)$ can be linearly extrapolated to geological ones $\left(10^{-12}\right.$ to $\left.10^{-14} \mathrm{~s}^{-1}\right)$. The flow laws (see Appendix A) related to these mechanisms are yet the only available mean to connect strain rate to stress for geological materials.

The model presented here aims to connect the evolution of rock textures with the underlying dominant creep mechanisms while keeping with the self-consistent framework of the grain damage theory. This is obtained by linking the theoretical evolution of the statistical variables described by our set equations to the observation and interpretation of natural mylonitic texture. To this end, it is crucial not only to measure and constrain the evolution of phases mean grain sizes during deformation and grain recovery but also to define and characterize a set of variable able to fully describe the variety of mylonite textures. To serve this purpose, the self-consistent physical framework provided by the Bercovici and Ricard (2012), Ricard and Bercovici (2009), and Rozel et al. (2011) grain damage theory appears to be of great efficiency. Indeed, the continuum approach using statistical variables allows to represent geological processes over a wide range of scales and statistical variables can easily be compared to natural or experimental measures.

In this model, we consider a set of five statistical variables to be sufficient in describing the microscopic texture characterizing a mylonitized two-phase aggregate. These variables are the two mean grain sizes $\mathcal{R}_{1}$ and $\mathcal{R}_{2}$, the mean interface curvature radius $r$ which depicts the overall coarseness of the interface area between the two phases and eventually the phases volumic fractions $\phi_{1}$ and $\phi_{2}=1-\phi_{1}$. If the whole grains-size distributions are taken into account, the assumption of self-similar distribution (Rozel et al., 2011) implies that all distributions parameters are only function of the mean $\mathcal{R}_{i}$, in this case the normalized variance $\sigma$ (normalized by the mean grain-size) also has to be fixed (see section B2). Even if, rigorously speaking, we do not account for the anisotropic fabric ubiquitous in ductile deformation, the "roughness" variable $\mathcal{P}_{i}$ could to some extent be regarded as a measure of grain shape variation (stretching and distortion of crystal surface), but we keep it constant in the current formalism.

\subsection{Zener Pinning, Phase Mixing, and Texture Evolution}

In dealing with two-phase aggregates, Bercovici and Ricard (2012) proposed a micromechanical model to account for the effect of interface in grain-size evolution, eventually yielding an equation where Zener pinning not only impedes grain recovery but also actively promotes grain-size reduction during deformation. We consider that the Zener pinning effect is only relevant to grain coarsening. This leads to a grain-size equation where the Zener coefficient $\mathcal{Z}_{i}$ appears only in the coarsening term of equations (112) and (113). We achieve this by proposing a partition function $\mathfrak{f}_{\alpha i}$ of the Zener coefficient $\mathcal{Z}_{i}$ (equation (110)).

Unlike previous studies (Bercovici \& Ricard, 2012), we do not assume entire independence of the interface density $\alpha$ on the two phases mean grain sizes $\mathcal{R}_{i}$. We use the Zener pinning mathematical description of Hillert (1965) and Bercovici and Ricard (2012) in order to propose an equation for $\alpha$ that depends on the full set of statistical variables $\left(\mathcal{R}_{1}, \mathcal{R}_{2}, r, \phi_{1}\right.$, and $\left.\sigma\right)$. Thus, each grain-size equation appears related on $r$ and vice versa. This dependence implies to modify the evolution equation for the interface mean curvature radius, as both coarsening and damage term appears now dependent on grain size. Furthermore, in spite of reliable knowledge of the related micromechanisms, we choose to allow for work storage in the diffusion creep domain. Within the general framework of this model, we show that such a mechanism requires a partition fraction at least 160 time the dislocation creep one in order to reach the steady-state grain size predicted by the Post and Tullis (1999) piezometer (Figure E6). The presented model allows for different phase rheologies and grain growth laws. As a consequence, an expression for the interface mean curvature radius coarsening rate $\hat{G}_{I}$ that is slightly different than the Bercovici and Ricard (2012) one is proposed (equations (145) and (146)). The resulting coarsening rate appears higher, but it does not significantly impacts the results.

In order to represent the textural evolution of a two-phase aggregate, we are especially interested in measuring the amount of mixing between the two phases. Indeed, the evolution of mixing (Figure E2) can be regarded as a critical clue to characterize both the active deformation mechanisms and the relative strength of the deforming material (Bercovici \& Skemer, 2017; Cross \& Skemer, 2017; Menegon et al., 2015; Precigout \& Stunitz, 2016). Taking into account the proposed set of variables, the degree of mixing of the aggregate and 
its evolution are described using the ratio between the interface area $\alpha$ and the sum of all grain boundaries within the control volume $\alpha_{11}+\alpha_{22}+\alpha$ (Heilbronner \& Barrett, 2014; see section 3.5.3 and equations (115) and (116)). This variable called $S$ measures the quantity of grain clusters and their relative sizes with respect to the mean grain sizes. Therefore, taking into account the equation for $\alpha(66)$ and $\alpha_{i}$ (15), the evolution of the mixing state between the phases is controlled by the difference between the grain size $\mathcal{R}_{i}$ and mean interface curvature radius $r$. Mixing requires the mean interface curvature $r$ to be smaller than the mean grain sizes, while phase segregation (or clustering, because formation of clusters requires intraphase grain boundaries creation) requires the mean grain sizes to be smaller than the mean interface curvature.

In the presented formalism, the dependence of the partition function $\mathfrak{f}_{\alpha i}$ implies that the damage term of the interface mean curvature radius equation is coupled to the grain-growth slow down (equation (111)). The evolution rates of these variables are directly controlled by the partition function $\chi_{i}$, which is imposed to be 1 in order to couple interface damage with Zener pinning and the interface coarsening rate $\hat{G}_{I}$. Therefore, constraining the forms or the values of $\chi_{i}$ requires to constrain the effect of deformation mechanisms on the textural characteristics of the aggregate. Accordingly, the grain damage and mixing that is depicted in Figure E8a results from this coupling. They are coherent with the effect of a nucleation mechanism on the aggregate (Precigout \& Stunitz, 2016) because nucleation would promote both the formation of very small grains and an important quantity of interface grain boundaries. However, here this effect relates on the Zener pinning coefficient promoting the damage of interface to the point where the Zener pinning itself becomes negative $\left(\mathcal{R}_{i}<r\right.$; see equation (57)) which cannot explicitly be related to nucleation.

\subsection{Thermodynamics and Grain-Size Distributions}

In the physical development of section 3, we use Onsager (1931) and Fischer et al. (2014) thermodynamical formalism to infer the relationship between the evolution equations. However, dealing with the full coupling between the variables describing a rock fabric and stress/strain-rate tensor requires our variable to be of the same tensorial order (De Groot \& Mazur, 1984).

In theory, the deformation work rate term of the entropy production equation should be written as the product of the strain rate (a thermodynamical flux) and the stress (a dissipation force; De Groot \& Mazur, 1984). However, because of the non-Newtonian rheology of mineral aggregates, the stress depends on the strain rate; therefore, the dissipation force is a function of the thermodynamical flux which requires a more general thermodynamical extremum principle. As a consequence, keeping with the hypothesis of linear and homogeneous evolution equations yields formally an unusual deformation work rate equation where the dissipation force has to be the deformation work rate while its conjugate thermodynamical flux is 1 (equations (74) and (95)). In such a framework, we show that equations equivalent than the ones of previous studies (Bercovici \& Ricard, 2012; Rozel et al., 2011) can be obtained.

Concerning the shapes of the grain-size distributions, we assume self-similar log-normal grain-size distributions (Rozel et al., 2011). This choice simplifies the mathematical treatment and remains reasonably consistent with natural observations in mono-phase (Faul \& Scott, 2006; Feltham, 1957; Slotemaker, 2006) and polyphase (Brodhag \& Herwegh, 2010; Dimanov et al., 2003) aggregates. Rozel et al. (2011) proposes to account for the effect of self-similar grain-size distribution (variance normalized by the mean) on the prefactor of diffusion creep rheological law (see section B2 and equation (A12)). However, experimental flow laws parameters already incorporate this effect (characteristic of the experimental aggregate). Therefore, allowing a variable variance in the model requires a correction of the $b_{i}$ parameter using the experimental variance of the aggregate used to constrain the rheological law (Table E2). As illustrated by Figure E5 (red-dashed line), taking into account this correction, the variance of the grain-sizes distribution has a nonnegligible impact on the aggregate rheology by shifting the steady-state mean grain size toward smaller sizes. When the mean grain size reaches the field boundary, most of the grains are already deforming in the diffusion creep regime, while in term of mass, the phase is still predominantly recrystallizing.

Eventually, upscaling this model from $0.1-1 \mathrm{~m}$ to shear-zone scales (100 $\mathrm{m}$ to $10 \mathrm{~km}$ ) requires to account for the temperature variations due to dissipative processes. Indeed, the energy not dissipated by grain-damage mechanisms has to be dissipated through heat. However, temperature increase as a result of deformation ("shear heating") is not expected to play a major role at the space/time scales that are in the scope of this study. Indeed, heat diffusion would be too efficient, and thermal dissipation cannot result in an effective viscosity drop (low Brinkmann number). Therefore, we do not treat heat sources and fluxes in this study. 
Indeed, due to the nonlinear dependence on temperature of rheological laws (Arrhenius), Onsager's linear formalism would not be sufficient and a nonlinear thermodynamical formalism required.

\section{Conclusion and Perspectives}

This study provides a model for the evolution of two-phase rock texture evolution during ductile deformation from the two-phase damage theory of Bercovici \& Ricard $(2012,2013)$. The coupling between mean grain size and interface density allows us to track the effect of microscopical deformation mechanism on the texture and to propose an equation to transfer this effect on the macroscopical scale. Grain-size relevant for ultramylonites could be reached either by considering the activation of a grain-size reduction mechanism in the diffusion creep domain or by the interaction between interface and intraphase grain boundaries area in two-phase aggregate (section 3.5.2). The evolution of phases mixing during long-term deformation at constant strain rate displays a complex global aggregate strength involving important stress weakening whose impact has to be investigated at larger scales. This rheology is likely to impact the initiation and dynamic of shear zones and to study the scale transfer of strain localization from shear bands to fully developed shear zones.

Even if the observation of natural and experimental rock samples provides meaningful insights on the relative behavior of the model variables in order to accurately represent the textural evolution of rock during deformation, a reliable set of quantified data is required to constrain the magnitude of the variables and their relationships. Measured mean grain sizes and phase proportions are generally available in the literature treating of rock microstructures, and grain-size distribution parameters appear to be increasingly included. However, there is few data available on interface and mixing quantification (Kruse \& Stunitz, 1999). In order to test the validity of our set of equations in accurately describing texture evolution during deformation, we will constrain their values and relations using natural mylonites samples. While not in the scope of the current model, the understanding of grain-size reduction mechanisms during diffusive creep requires a micromechanical model in order to link the magnitude of the partition fraction $\mathfrak{f}_{N i}$ to the aggregate fabric, intensive parameters, and deformational work rate.

In mylonitized rocks, the microstructural fabric displays a strong anisotropy characteristic of the magnitudes and orientations of principal stresses. However, in the current state of the model, considering scalar variables implies textural isotropy. Acknowledging for the strong anisotropy observed in natural samples thus requires to consider tensorial variables accounting for textural variations in all space directions. This is also required to self-consistently link the geometry of mylonite texture to the stress and strain-rate tensors.

The model of textures evolution proposed here will be implemented in a 2-D framework to study its impact on strain localization and ductile deforming rock dynamic at higher scales. Indeed, the framework provided by the Bercovici and Ricard (2012) theory allows to study the effect of scale transfer between the length scale on which the statistical variables are averaged (the volume characteristic size represented by the averaged variables) and the length scale of the geodynamical model where each point yields an effective viscosity that depends on the statistical variables. Therefore, numerical results could be discussed with respect to natural rocks at two different scales which could provide efficient mean to both constraint the physics of the model and the effect of textural variables on the evolution of the whole material rheology.

\section{Appendix A: Mass Conservation}

According to Bercovici and Ricard (2012), considering the two phases to fill the entire control volume (no gap or void of any king the grains), the two phases incompressible, and no mass transfer between the two phases, the mass conservation equations reads

$$
\frac{\partial \phi_{i}}{\partial t}+\nabla \cdot\left(\phi_{i} \mathbf{v}_{i}\right)=0
$$

where the term $\mathbf{v}_{i}$ accounting for phase velocity could be potentially different for each phase. However, as stated in Bercovici and Ricard (2012), in presence of two solid silicates phases, the relative difference between $\mathbf{v}_{1}$ and $\mathbf{v}_{2}$ is considered negligible so that $\mathbf{v}_{1}=\mathbf{v}_{2}=\mathbf{v}$. Therefore, the velocity averaged over the control volume is $\overline{\mathbf{v}}=\sum_{i} \phi_{i} \mathbf{v}_{i}=\mathbf{v}$, and there is only one mass conservation equation that yields

$$
\frac{\partial \phi}{\partial t}+\mathbf{v} \cdot \nabla \phi=0 \quad \text { and } \quad \nabla \cdot \mathbf{v}=0
$$


where $\phi$ could be indifferently $\phi_{1}$ or $\phi_{2}$. In a finite volume $\Delta V$, the number of grain between sizes $\mathrm{R}_{1}$ and $\mathrm{R}_{2}$ is

$$
\mathrm{n}_{i}=\int_{\Delta V} \int_{\mathrm{R}_{1}}^{\mathrm{R}_{2}} v_{i} \mathrm{dR} \phi_{i} \mathrm{~d} V .
$$

$v_{i}$ being the grain-size distribution function. The control volume $\Delta V$ is considered open and fixed, and in the same conditions, the portion of grain-size space between two grain sizes $R_{1}$ and $R_{2}$ is fixed and open to mass transfer from other populations in the grain-size space. Therefore, the rate of change of grains number reads

$$
\begin{aligned}
\frac{\partial \mathrm{n}_{i}}{\partial t} & =\int_{\Delta V} \int_{\mathrm{R}_{1}}^{\mathrm{R}_{2}} \frac{\partial v_{i} \phi_{i}}{\partial t} \mathrm{dRd} V \\
& =\int_{\Delta A} \int_{\mathrm{R}_{1}}^{\mathrm{R}_{2}} v_{i} \mathrm{dR} \phi_{i} \mathbf{v}_{i} \cdot \hat{\mathbf{n}} \mathrm{d} A-\int_{\Delta V}\left[v_{i} \dot{\mathrm{R}}_{i}\right]_{\mathrm{R}_{1}}^{\mathrm{R}_{2}} \phi_{i} \mathrm{~d} V+\int_{\Delta V} \int_{\mathrm{R}_{1}}^{\mathrm{R}_{2}} \Gamma_{i} \mathrm{dR} \phi_{i} \mathrm{~d} V,
\end{aligned}
$$

where $\Delta A$ is the surface area of the volume $\Delta V, \hat{\mathbf{n}}$ is the unit vector normal to a surface element $\mathrm{d} A, \dot{\mathrm{R}}_{i}$ is the growth rate of a grain of size $\mathrm{R}$, and $\Gamma_{i}$ is the discontinuous transfer of populations dispersed within the grain-size distribution resulting of breaking or fusing of grains. Considering the limit where $R_{2}-R_{1} \rightarrow 0$, taking in account that $v_{i}$ and $\phi_{i}$ are independent from the grain-size $\mathrm{R}$ and using Stokes theorem yields the distribution continuity equation (Bercovici \& Ricard, 2012, appendix B, equation, B.11):

$$
\frac{\partial \phi_{i} v_{i}}{\partial t}+\nabla \cdot\left(\mathbf{v}_{i} \phi_{i} v_{i}\right)+\phi_{i} \frac{\partial}{\partial \mathrm{R}}\left(\dot{\mathrm{R}}_{i} v_{i}\right)=\Gamma_{i} \phi_{i}
$$

which, using the equation (A1), could be written

$$
\frac{D_{i} v_{i}}{D t}+\frac{\partial}{\partial \mathrm{R}}\left(\dot{\mathrm{R}}_{i} v_{i}\right)=\Gamma_{i}
$$

with the differential operator:

$$
\frac{D_{i}}{D t}=\frac{\partial}{\partial t}+\mathbf{v}_{i} \cdot \nabla
$$

From there, it is possible to write the evolution of any grain property $\breve{\theta}_{i}$ :

$$
\begin{aligned}
\frac{D_{i} \Theta_{i}}{D t} & =\int_{\Delta V} \int_{0}^{\infty}\left(v_{i}\left[\frac{D_{i} \breve{\theta}_{i}}{D t}+\dot{\mathrm{R}} \frac{\partial \breve{\theta}_{i}}{\partial \mathrm{R}}\right]-\frac{\partial}{\partial \mathrm{R}}\left(\dot{\mathrm{R}}_{i} \breve{\theta}_{i} v_{i}\right)+\breve{\theta}_{i} \Gamma_{i}\right) \mathrm{dR} \phi_{i} \mathrm{~d} V \\
& =\int_{\Delta V} \int_{0}^{\infty}\left(v_{i} \frac{d_{i} \breve{\theta}_{i}}{\mathrm{~d} t}-\frac{\partial}{\partial \mathrm{R}}\left(\dot{\mathrm{R}}_{i} \breve{\theta}_{i} v_{i}\right)+\breve{\theta}_{i} \Gamma_{i}\right) \mathrm{dR} \phi_{i} \mathrm{~d} V
\end{aligned}
$$

defining the full derivative in grain-size space:

$$
\frac{d_{i}}{\mathrm{~d} t}=\frac{D_{i}}{D t}+\dot{\mathrm{R}}_{i} \frac{\partial}{\partial \mathrm{R}}
$$

Given the fact the distribution must vanish at a definite maximum and minimum grain size (i.e., $\dot{\mathrm{R}}_{i} \breve{\theta}_{i} v_{i} \rightarrow 0$ if $\mathrm{R} \rightarrow 0$ or $\infty$ ) and that $\breve{\theta}_{i}$ is an extensive property then equation (A10) yields

$$
\frac{D_{i} \Theta_{i}}{D t}=\int_{\Delta V} \int_{0}^{\infty}\left(v_{i} \frac{d_{i} \breve{\theta}_{i}}{\mathrm{~d} t}+\Gamma_{i} \breve{\theta}_{i}\right) \mathrm{dR} \phi_{i} \mathrm{~d} V .
$$

Written in term of the grain mass, it reads

$$
\frac{D_{i} \mathrm{M}_{i}}{D t}=\int_{\Delta V} \int_{0}^{\infty}\left(v_{i} \frac{d_{i} \breve{\mathrm{m}}_{i}}{\mathrm{~d} t}+\Gamma_{i} \breve{\mathrm{m}}_{i}\right) \mathrm{dR} \phi_{i} \mathrm{~d} V,
$$

where $\breve{\mathrm{m}}_{i}$ is the mass of one grain and $\mathrm{M}_{i}$ the total mass of phase $i$ in the volume $\Delta V$. The volume $\Delta V$ is closed, assuming that locally within its limits, mass is conserved, thus $\frac{D_{i} \mathrm{M}_{i}}{D t}=0$ which implies

$$
\int_{0}^{\infty}\left(v_{i} \frac{d_{i} \breve{\mathrm{m}}_{i}}{\mathrm{~d} t}+\Gamma_{i} \breve{\mathrm{m}}_{i}\right) \mathrm{dR}=0 .
$$


So that, the sum of discontinuous and continuous mass transport within the volume $\Delta V$ must satisfy mass conservation. Furthermore, following Ricard and Bercovici (2009), it is assumed that continuous processes (grains growing or shrinking) and discontinuous ones (breaking or fusing of grains) are decoupled:

$$
\begin{gathered}
\int_{0}^{\infty} \frac{d_{i} \breve{\mathrm{m}}_{i}}{\mathrm{~d} t} v_{i} \mathrm{dR}=0, \\
\int_{0}^{\infty} \Gamma_{i} \breve{\mathrm{m}}_{i} \mathrm{dR}=0 .
\end{gathered}
$$

A general differential operator is defined as

$$
\frac{\mathfrak{D}_{i}}{\mathfrak{D} t}=\frac{d_{i}}{\mathrm{~d} t}+\frac{\Gamma_{i}}{v_{i}} \equiv \frac{D_{i}}{D t}+\dot{\mathrm{R}}_{i} \frac{\partial}{\partial \mathrm{R}}+\frac{\Gamma_{i}}{v_{i}},
$$

so that equations (A12) and (A13) read:

$$
\begin{gathered}
\frac{D_{i} \Theta_{i}}{D t}=\int_{\Delta V} \int_{0}^{\infty} \frac{\mathfrak{D}_{i} \breve{\theta}_{i}}{\mathrm{~d} t} v_{i} \mathrm{dR} \phi_{i} \mathrm{~d} V, \\
\frac{D_{i} \mathrm{M}_{i}}{D t}=\int_{\Delta V} \int_{0}^{\infty} \frac{\mathfrak{D}_{i} \breve{\mathrm{m}}_{i}}{\mathrm{~d} t} v_{i} \mathrm{dR} \phi_{i} \mathrm{~d} V=0 .
\end{gathered}
$$

\section{Appendix B: Grain-Size Distributions}

\section{B1. Self-Similar Grain-Sizes Distributions}

The grain-sizes distribution $\left(v_{i}\right)$ evolution is calculated using the same self-similarity hypothesis than Rozel et al. (2011). Self-similarity implies that the distribution shape remains similar throughout deformation. As a consequence, all the distribution moments (mean, variance, skewness, etc.) are functions of a mean grain-size $\mathcal{R}_{i}$ which is time dependent. If this assumption is valid for grain growth in the limit of long times, there is qualitative arguments to maintain it even in the presence of deformation. Indeed, small grains should be less sensitive to deformation than bigger ones. Consequently, the distribution variance and mean are driven down simultaneously. Rozel et al. (2011) defines the self-similar grain-size distribution $v_{i}$ as

$$
v_{i}(\mathrm{R}, \mathbf{x}, t)=B\left(\mathcal{R}_{i}(\mathrm{t})\right) H(u),
$$

where $\mathcal{R}_{i}$ is the time-dependent characteristic mean grain size of phase $i, u=\mathrm{R} / \mathcal{R}_{i}$ is the self-similarity variable (assumed identical between the two phase for the sake of simplicity even if it is not truly exact), $B\left(\mathcal{R}_{i}\right)$ an amplitude function, and $H(u)$ the shape function of the distribution. Taking into account the assumption that $\mathbf{v}_{i}=\tilde{\mathbf{v}}=\mathbf{v}$, all the material derivatives become equivalent to $D / D t=\partial / \partial t+\mathbf{v}$. $\nabla$. Therefore, all variables that are function of both time $t$ and space $\mathbf{x}$ become function of time $t$ following a particle with a velocity $\mathbf{v}$, that is, $f(\mathrm{R}, \mathbf{x}, t)=f(\mathrm{R}, \mathrm{t})$. Using equation (6),

$$
\int_{0}^{\infty} \breve{V} v_{i} \mathrm{dR}=1
$$

The amplitude $B$ can be directly derived:

$$
\int_{0}^{\infty} \breve{V} \nu_{i} \mathrm{dR}=\frac{4}{3} \pi \mathcal{R}_{i}^{4} B\left(\mathcal{R}_{i}\right) \int_{0}^{\infty} u^{3} H(u) \mathrm{d} u=1,
$$

yielding

$$
B=\frac{3}{4 \pi \lambda_{3} \mathcal{R}_{i}^{4}}
$$

In this equation the term $\lambda_{3}$ is the third moment of the grain-size distribution, defined according to Rozel et al. (2011) as

$$
\lambda_{n}=\int_{0}^{\infty} u^{n} H(u) \mathrm{d} u
$$


for $n=3$. Accordingly, the distribution $v_{i}$ yields from equations (B1) and (B4):

$$
v_{i}=\frac{3 H(u)}{4 \pi \lambda_{3} \mathcal{R}_{i}^{4}} .
$$

Derived with respect to time variable, it yields

$$
\frac{D v_{i}}{D t}=\frac{\partial \nu_{i}}{\partial t}=-\frac{3}{4 \pi \lambda_{3} \mathcal{R}_{i}^{5}} \frac{\mathrm{d} \mathcal{R}_{i}}{\mathrm{~d} t} \frac{1}{u^{3}} \frac{d\left(u^{4} H\right)}{\mathrm{d} u} .
$$

Rozel et al. (2011) demonstrated that the form of $D v_{i} / D t$ must constrain the form of $\dot{\mathrm{R}}_{i}$ and $\Gamma_{i}$ because they have to be symmetrical in order to be balanced in equation (A7), thus leading to

$$
\Gamma_{i}=\frac{3}{4 \pi \lambda_{3} \mathcal{R}_{i}^{5}} \mathcal{D}\left(\mathcal{R}_{i}\right) \frac{1}{u^{3}} \frac{d\left(u^{4} H\right)}{\mathrm{d} u},
$$

where $D\left(\mathcal{R}_{i}\right)$ is an amplitude function of $\mathcal{R}_{i}$ accounting for discontinuous fluxes of grains populations. Symmetrically, the $\frac{\partial\left(\dot{\mathrm{R}}_{i} v_{i}\right)}{\partial \mathrm{R}}$ term reads

$$
\frac{\partial\left(\dot{\mathrm{R}}_{i} v_{i}\right)}{\partial \mathrm{R}}=\frac{3}{4 \pi \lambda_{3} \mathcal{R}_{i}^{5}} C\left(\mathcal{R}_{i}\right) \frac{1}{u^{3}} \frac{d\left(u^{4} H\right)}{\mathrm{d} u},
$$

which after integration yields

$$
\dot{\mathrm{R}}_{i}=C\left(\mathcal{R}_{i}\right)\left(\frac{b}{H}+u+\frac{3}{H} \int_{0}^{u} H\left(u^{\prime}\right) \mathrm{d} u^{\prime}\right),
$$

where $C\left(\mathcal{R}_{i}\right)$ is the amplitude function of $\mathcal{R}_{i}$ that account for the continuous fluxes of grains populations. Substituting equations (B7)-(B9) into the equation (A7), Rozel et al. (2011) proposes the mean grain-size kinetic equation:

$$
\frac{\mathrm{d} \mathcal{R}_{i}}{\mathrm{~d} t}=C\left(\mathcal{R}_{i}\right)-D\left(\mathcal{R}_{i}\right)
$$

\section{B2. Log-Normal Grain-Size Distributions}

To explore the model behavior, we need to choose the form of the self-similar distribution. A log-normal distribution function, that is, a Gaussian distribution when plotted in logarithmic coordinates, appears to be a reasonable approximation for natural grain-size distributions, considering the mean of the grain-size distribution plotted in logarithmic coordinate as the "mean grain size" $\mathcal{R}_{i}$ (Rozel et al., 2011). The self-similar distribution reads

$$
H(u)=\frac{1}{\sqrt{2 \pi} \sigma u} e^{-\log (u)^{2} /\left(2 \sigma^{2}\right)},
$$

where $u$ is the self-similar variable $\mathrm{R} / \mathcal{R}_{i}$, and $\sigma$ is the dimensionless variance of the distribution plotted as a function of $\ln \left(\mathrm{R} / \mathcal{R}_{i}\right)$. This choice yields the following important quantity from equation (B5):

$$
\lambda_{n}=\int_{0}^{\infty} u^{n} H(u) \mathrm{d} u=\exp \left(\frac{n^{2} \sigma^{2}}{2}\right) .
$$

The true mean grain size reads

$$
\langle\mathrm{R}\rangle=\frac{\int_{0}^{\infty} \mathrm{R} v(\mathrm{R}) \mathrm{dR}}{\int_{0}^{\infty} v(\mathrm{R}) \mathrm{dR}}=\mathcal{R}_{i} \exp \left(\frac{\sigma^{2}}{2}\right),
$$

and the variance function of $\mathcal{R}_{i}$ yields

$$
\sigma^{2}\left(\mathcal{R}_{i}\right)=\mathcal{R}_{i}^{2} \exp \left(\sigma^{2}\right)\left[\exp \left(\sigma^{2}\right)-1\right]
$$

\section{Appendix C: Thermodynamics of Two-Phase Aggregates}

\section{C1. Energy}

Using the framework described above, the rate of total energy (where the volume kinetic energy is neglected) derived by Bercovici and Ricard (2012) and Ricard and Bercovici (2009) reads

$$
\frac{\partial E}{\partial t}=-\mathrm{J}_{\varepsilon}-\mathrm{J}_{\gamma}+W_{b}+W_{s}+W_{l}-\int_{\delta A} q \mathrm{~d} A+\int_{\delta V} Q \mathrm{~d} V,
$$


with

1. $\mathrm{J}_{\varepsilon}$ : energy flux due to grain internal energy and surface tension of grain boundaries;

2. $\mathrm{J}_{\gamma}$ : energy flux due to surface tension of interfaces between phases;

3. $W_{b}$ : work done by body forces;

4. $W_{s}$ : work done by surface forces;

5. $W_{l}$ : work done by line forces;

6. $q$ : surface heat flux; and

7. $Q$ : heat source.

The Gibbs energy written for a grain reads (Bercovici \& Ricard, 2012, appendix E, equation E.6):

$$
\frac{d_{i} \breve{\varepsilon}_{i}}{\mathrm{~d} t}=T \frac{d_{i} \breve{S}_{i}}{\mathrm{~d} t}-\breve{P}_{i} \frac{d\left(1 / \rho_{i}\right)}{\mathrm{d} t} .
$$

$\breve{\varepsilon}$ is the grain specific internal energy, $\breve{S}_{i}$ is the grain specific entropy, $T$ is the temperature within the control volume, $P_{i}$ the pressure in phase $i$, and $\rho_{i}$ the phase $i$ density. The chemical potential of a grain is (Bercovici \& Ricard, 2012, appendix E, equation E.7):

$$
\breve{\mu}_{i}=\breve{\varepsilon}_{i}-T \breve{S}_{i}+\breve{P}_{i} \rho_{i}
$$

Taking into account that

1. the temperature $T$ is assumed homogeneous across all grains of all phases within the control volume $\Delta V$.

2. both phases are incompressible which leads to $\frac{\mathrm{d} \rho_{i}}{\mathrm{~d} t}=0$ and $\mathrm{d} \breve{\varepsilon}_{i}=T \mathrm{~d} \breve{S}_{i}$ and applying expansion of scale yields $\breve{\varepsilon}_{i}=T \breve{S}_{i}$ and $\breve{\mu}_{i}=\breve{P}_{i} / \rho_{i}$ (Bercovici \& Ricard, 2012, appendix E).

3. the velocities of both phases are assumed very close, that is, $\mathbf{v}_{1}=\mathbf{v}_{2}=\tilde{\mathbf{v}}=\mathbf{v}$.

4. the effective pressure $\Pi_{i}=P_{i}+\gamma_{i} \mathcal{K}_{i}$ includes the work done by grain boundary surface tension on the surface of the control volume $\left(\mathcal{K}_{i}\right.$ being the average grain-boundary curvature of phase $i$; Bercovici \& Ricard, 2012, appendix D).

5. the effective pressure difference $\Delta \Pi$ is defined as $\Delta \Pi=\Pi_{2}-\Pi_{1}$ (Bercovici \& Ricard, 2012, appendix D).

6. the effective interface surface tension is defined as $\gamma_{I}=\tilde{\gamma}_{I}-\sum_{i} \phi_{i} \gamma_{i}$ with $\tilde{\gamma}_{I}$ being the interface surface tension energy and $\gamma_{i}$ the intraphase grain boundaries surface tension energy (Bercovici \& Ricard, 2012, appendix E.1).

7. the phases volume fraction is defined as $\phi=\phi_{1}=1-\phi_{2}$ (Bercovici \& Ricard, 2012, appendix D).

and using standard method (see; Ricard \& Bercovici, 2009 and; Bercovici \& Ricard, 2012, appendix E.1 for details), the equation ( $\mathrm{C} 1$ ) eventually reduces to

$$
\begin{aligned}
\sum_{i} \int_{0}^{\infty}\left(T \frac{\mathfrak{D}_{i}\left(\breve{m}_{i} \breve{\varepsilon}_{i}\right)}{\mathfrak{D} t}-\breve{P}_{i} \frac{\mathfrak{D}_{i}\left(\breve{m}_{i} / \rho_{i}\right)}{\mathfrak{D} t}+\breve{\mu}_{i} \frac{\mathfrak{D}_{i} \breve{m}_{i}}{\mathfrak{D} t}+\gamma_{i} \frac{\mathfrak{D}_{i} \breve{\mathcal{A}}_{i}}{\mathfrak{D} t}\right) v_{i} \mathrm{dR}+\gamma_{I} \frac{\tilde{D} \alpha}{\tilde{D} t} \\
=c(\Delta \mathbf{v})^{2}+\psi^{*}-\Delta \Pi \frac{\tilde{D} \phi}{\tilde{D} t}+Q-\nabla . \mathbf{q}
\end{aligned}
$$

where $\breve{m}_{i}$ is the mass of grain of phase $i, c(\Delta v)^{2}$ is a drag coefficient depending on the phases velocities, $Q$ is the heat source within the control volume, $\nabla . q$ is the heat fluxes across the control volume boundaries, and the differential operator $\mathfrak{D} / \mathfrak{D} t$ is the material derivative in the framework of phase $i$ defined by (A15):

$$
\frac{\mathfrak{D}_{i}}{\mathfrak{D} t}=\frac{d_{i}}{\mathrm{~d} t}+\frac{\Gamma_{i}}{v_{i}},
$$

and the differential operator $\tilde{D} / \tilde{D} t$ is the material derivative in the framework of interface defined by the equation:

$$
\frac{\tilde{D}}{\tilde{D} t}=\sum_{i} \omega_{i} \frac{D}{D t}
$$

The coefficient $\omega_{i}$ accounts for how the interfacial surface energy is partitioned between each phase (see; Bercovici et al., 2001; Bercovici \& Ricard, 2003, 2012, appendix D). 


\section{C2. Rate of Entropy Production}

The energy balance equation, as well as the Second Law of Thermodynamics, allows to infer the entropy production rate. As this production must inevitably be positive in the case of irreversible work, it becomes possible to use nonequilibrium thermodynamics to infer phenomenological laws and damage relations (De Groot \& Mazur, 1984). Following Bercovici and Ricard (2012, appendix E.2), the total entropy of the control volume $\Delta V$ is

$$
S=\int_{\Delta V} \sum_{i} \phi_{i} \int_{0}^{\infty} \breve{m}_{i} \breve{S}_{i} v_{i} \mathrm{dRd} V
$$

The Second Law of Thermodynamics states that entropy production must be equal or greater than to zero which is written as

$$
\frac{D S}{D t}=\int_{\Delta V} \sum_{i} \int_{0}^{\infty} \frac{\mathfrak{D}_{i}\left(\breve{m}_{i} \breve{S}_{i}\right)}{\mathfrak{D} t} v_{i} \mathrm{~d} \operatorname{Rd} V \geqslant-\int_{\Delta A} \frac{1}{T} \boldsymbol{q} \cdot \hat{\boldsymbol{n}} \mathrm{d} A,
$$

where the last term, on the left-hand side of the inequality, represents the entropy flux through the surface of the control volume. Therefore, using the equation (C3) divided by $T$ which is assumed uniform at every points of the control volume, the entropy production $\Sigma$ reads

$$
\begin{aligned}
\Sigma= & \sum_{i} \phi_{i} \int_{0}^{\infty}\left(\left(\breve{P}_{i} / \rho_{i}-\breve{\mu}_{i}\right) \frac{\mathfrak{D}_{i} \breve{m}_{i}}{\mathfrak{D} t}-\gamma_{i} \frac{\mathfrak{D}_{i} \breve{\mathcal{A}}_{i}}{\mathfrak{D} t}\right) v_{i} \mathrm{dR}-\gamma_{I} \frac{\tilde{D} \alpha}{\tilde{D} t}-\Delta \Pi \frac{\tilde{D} \phi_{1}}{\tilde{D} t} \\
& c(\Delta \nu)^{2}+\psi^{*}+Q-\frac{1}{T} \boldsymbol{q} \cdot \nabla T \geqslant 0,
\end{aligned}
$$

where $\breve{\mathcal{A}_{i}}$ is the boundary area of a grain of size $\breve{\mathrm{R}}$ defined by equation (3). According to the assumptions made for equation (C3), that is, $\breve{\mu}_{i}=\breve{P}_{i} / \rho_{i}$, equation (C9) becomes

$$
\Sigma=-\sum_{i} \phi_{i} \gamma_{i} \int_{0}^{\infty} \frac{\mathfrak{D}_{i} \breve{A}_{i}}{\mathfrak{D} t} \nu_{i} \mathrm{dR}-\gamma_{I} \frac{\tilde{D} \alpha}{\tilde{D} t}-\Delta \Pi \frac{\tilde{D} \phi_{1}}{\tilde{D} t}+c(\Delta v)^{2}+\psi^{*}+Q-\frac{1}{T} \boldsymbol{q} \cdot \nabla T \geqslant 0,
$$

which is the relation used by Bercovici and Ricard (2012) to derive phenomenological laws plus the heat source and fluxes terms. This equation describes the different entropy sources related to intraphase grain boundaries, interfaces, phases volumic fractions, deformational work, heat production, and fluxes. The drag work term $c\left(\Delta v_{i}\right)^{2}$ will be neglected as we expect two mineral phases to have macroscopic velocities of the same order (Bercovici \& Ricard, 2012). At the scales involved in the following model, the heat diffusion is not expected to play a major role; therefore, we neglect the heat production and fluxes terms.

\section{C2.1. Intraphase Grain Boundary Entropy Production Terms}

Using the expression of the effective grain area $\mathcal{A}_{i}$ (see equation (3)):

$$
\breve{\mathcal{A}}_{i}=\breve{\mathrm{A}}_{i} \breve{\mathrm{P}}_{i}=4 \pi \breve{\mathrm{R}}_{i}^{2} \breve{\mathrm{P}}_{i}
$$

and the definition of the $\mathfrak{D} / \mathfrak{D} t$ operator (equation (C5)), the first member of equation (C10) yields

$$
\begin{aligned}
&-\sum_{i} \phi_{i} \gamma_{i} \int_{0}^{\infty} \frac{\mathfrak{D} \breve{\mathcal{A}}_{i}}{\mathfrak{D} t} v_{i} \mathrm{dR}=-\sum_{i} \phi_{i} \gamma_{i}\left(\int_{0}^{\infty} \frac{\mathrm{d} \breve{\mathcal{A}}_{i}}{\mathrm{~d} t} \nu_{i} \mathrm{dR}+\int_{0}^{\infty} \breve{\mathcal{A}}_{i} \Gamma_{i} \mathrm{dR}\right) \\
&=-\sum_{i} \phi_{i} \gamma_{i}\left(\int_{0}^{\infty} \frac{\mathrm{d} \breve{A}_{i} \breve{\mathrm{P}}_{i}}{\mathrm{~d} t} v_{i} \mathrm{dR}+\int_{0}^{\infty} \breve{A}_{i} \breve{\mathrm{P}}_{i} \Gamma_{i} \mathrm{dR}\right) \\
&=-\sum_{i} \phi_{i} \gamma_{i}\left[\int_{0}^{\infty}\left(\breve{\mathrm{P}}_{i}+\breve{A}_{i} \frac{\mathrm{d} \breve{\mathrm{P}}_{i}}{\mathrm{~d} \breve{A}_{i}}\right) \frac{\mathrm{d} \breve{A}_{i}}{\mathrm{~d} t} v_{i} \mathrm{dR}+\int_{0}^{\infty} \breve{A}_{i} \breve{\mathrm{P}}_{i} \Gamma_{i} \mathrm{dR}\right] \\
&=-\sum_{i} \phi_{i} \gamma_{i}\left[-\int_{0}^{\infty}\left(\breve{\mathrm{P}}_{i}+\breve{A}_{i} \frac{\mathrm{d} \breve{\mathrm{P}}_{i}}{\mathrm{~d} \breve{A}_{i}}\right) \frac{\partial \dot{\mathrm{R}}_{i} v_{i}}{\partial \mathrm{R}} \breve{A}_{i} \mathrm{dR}\right. \\
&\left.+\int_{0}^{\infty} \breve{A}_{i} \breve{\mathrm{P}}_{i} \Gamma_{i} \mathrm{dR}\right] .
\end{aligned}
$$


Then, using equations (B8) and (B9) for the continuous $\left(\partial \dot{\mathrm{R}} v_{i} / \partial \breve{\mathrm{R}}\right)$ and discontinuous $\left(\Gamma_{i}\right)$ fluxes of grains in the grain-size space, respectively, the entropy production terms related to intraphase grain-boundary evolution eventually reads

$$
\begin{gathered}
-\sum_{i} \phi_{i} \gamma_{i} \int_{0}^{\infty} \frac{\mathfrak{D} \breve{\mathcal{A}}_{i}}{\mathfrak{D} t} v_{i} \mathrm{dR}=\sum_{i}\left[\frac{3 \phi_{i} \gamma_{i} C\left(\mathcal{R}_{i}\right)}{\lambda_{3} \mathcal{R}_{i}^{2}} \int_{0}^{\infty}\left(\breve{\mathrm{P}}_{i}+\breve{A}_{i} \frac{\mathrm{d} \breve{\mathrm{P}}_{i}}{\mathrm{~d} \breve{A}_{i}}\right) \frac{1}{u} \frac{d\left(u^{4} H\right)}{\mathrm{d} u} \mathrm{~d} u\right. \\
\left.-\frac{3 \phi_{i} \gamma_{i} D\left(\mathcal{R}_{i}\right)}{\lambda_{3} \mathcal{R}_{i}^{2}} \int_{0}^{\infty} \frac{\breve{\mathrm{P}}_{i}}{u} \frac{d\left(u^{4} H\right)}{\mathrm{d} u} \mathrm{~d} u\right],
\end{gathered}
$$

where we recall that $u=\breve{\mathrm{R}} / \mathcal{R}_{i}$ is the self-similarity variable, and $H(u)$ and $\lambda_{n}$ are, respectively, the shape function and the $n$th moment of the self-similar distribution (see Appendix B and equations (B12) and (B13)).

\section{C2.2. Interface Entropy Production Term}

From the entropy production equation (C10), given that $\frac{\tilde{D}}{\tilde{D} t}=\sum_{i} \omega_{i} \frac{\mathrm{d}}{\mathrm{d} t}$ is the material derivative related to phase interface, using the fact that $\sum_{i} \omega_{i}=1$ (see; Bercovici \& Ricard, 2012, appendix D), and using equation (B11), the interface density term reads

$$
\begin{aligned}
-\gamma_{I} \frac{\tilde{D} \alpha}{\tilde{D} t} & =-\gamma_{I}\left(\sum_{i} \frac{\partial \alpha}{\partial \mathcal{R}_{i}} \frac{\partial \mathcal{R}_{i}}{\partial t}+\frac{\partial \alpha}{\partial r} \frac{\partial r}{\partial t}+\frac{\partial \alpha}{\partial \phi_{1}} \frac{\partial \phi_{1}}{\partial t}\right) \\
& =-\gamma_{I}\left(\sum_{i} \frac{\partial \alpha}{\partial \mathcal{R}_{i}}\left[C\left(\mathcal{R}_{i}\right)-D\left(\mathcal{R}_{i}\right)\right]+\frac{\partial \alpha}{\partial r} \frac{\partial r}{\partial t}+\frac{\partial \alpha}{\partial \phi_{1}} \frac{\partial \phi_{1}}{\partial t}\right) .
\end{aligned}
$$

\section{C2.3. Final Entropy Production Equation}

Substituting the expressions (C13) and (C15) in (C10) and factorizing yields the entropy production equation:

$$
\begin{gathered}
\Sigma=\sum_{i}\left\{\left[\frac{3 \phi_{i} \gamma_{i}}{\lambda_{3} \mathcal{R}_{i}^{2}} \int_{0}^{\infty}\left(\breve{\mathrm{P}}_{i}+\breve{A}_{i} \frac{\mathrm{d} \breve{\mathrm{P}}_{i}}{\mathrm{~d} \breve{A}_{i}}\right) \frac{1}{u} \frac{d\left(u^{4} H\right)}{\mathrm{d} u} \mathrm{~d} u-\gamma_{I} \frac{\partial \alpha}{\partial \mathcal{R}_{i}}\right] C\left(\mathcal{R}_{i}\right)\right. \\
\left.-\left[\frac{3 \phi_{i} \gamma_{i} D\left(\mathcal{R}_{i}\right)}{\lambda_{3} \mathcal{R}_{i}^{2}} \int_{0}^{\infty} \frac{\breve{\mathrm{P}}_{i}}{u} \frac{d\left(u^{4} H\right)}{\mathrm{d} u} \mathrm{~d} u-\gamma_{I} \frac{\partial \alpha}{\partial \mathcal{R}_{i}}\right] D\left(\mathcal{R}_{i}\right)\right\}-\gamma_{I} \frac{\partial \alpha}{\partial r} \frac{\partial r}{\partial t} \\
-\left(\gamma_{I} \frac{\partial \alpha}{\partial \phi_{1}}+\Delta \Pi\right) \frac{\tilde{D} \phi_{1}}{\tilde{D} t}+\psi^{*} .
\end{gathered}
$$

For the sake of simplicity, we then choose to assume that the grain roughness variable $\breve{\mathrm{P}}_{i}$ remains independent from the grain-size variable $\breve{\mathrm{R}}_{i}$ and constant with respect to time, that is, all the grains of phase $i$ share the same shape, or the shape of a grain does not depend on its size. Therefore, the variable $\breve{P}_{i}$ is considered in the following model as a mean parameter $\mathcal{P}_{i}$ representing the average "shape" of the grains. As a consequence, the grain roughness $\breve{\mathrm{P}}_{i}$ is not different from the $\Pi_{i}$ coefficient proposed by Ricard and Bercovici (2009, section 2.2) to account for averaged grain shapes. However, we retain the variable $\breve{P}_{i}$ in the current model. This simplification yields from (C16):

$$
\begin{aligned}
\Sigma=\sum_{i}\{ & {\left[\frac{3 \phi_{i} \gamma_{i} \mathcal{P}_{i}}{\lambda_{3} \mathcal{R}_{i}^{2}} \int_{0}^{\infty} \frac{1}{u} \frac{d\left(u^{4} H\right)}{\mathrm{d} u} \mathrm{~d} u-\gamma_{I} \frac{\partial \alpha}{\partial \mathcal{R}_{i}}\right] C\left(\mathcal{R}_{i}\right) } \\
& \left.-\left[\frac{3 \phi_{i} \gamma_{i} \mathcal{P}_{i}}{\lambda_{3} \mathcal{R}_{i}^{2}} \int_{0}^{\infty} \frac{1}{u} \frac{d\left(u^{4} H\right)}{\mathrm{d} u} \mathrm{~d} u-\gamma_{I} \frac{\partial \alpha}{\partial \mathcal{R}_{i}}\right] D\left(\mathcal{R}_{i}\right)\right\} \\
& -\gamma_{I} \frac{\partial \alpha}{\partial r} \frac{\partial r}{\partial t}-\left(\gamma_{I} \frac{\partial \alpha}{\partial \phi_{1}}+\Delta \Pi\right) \frac{\tilde{D} \phi_{1}}{\tilde{D} t}+\psi^{*},
\end{aligned}
$$

which for log-normal distributions (see section B2) and using equations (B12) and (B13) allow to evaluate the integrand through integration by parts (Rozel et al., 2011):

$$
\begin{aligned}
\int_{0}^{\infty}\left(\frac{1}{u} \frac{d\left(u^{4} H\right)}{\mathrm{d} u}\right) \mathrm{d} u & =\left[u^{3} H(u)\right]_{0}^{\infty}+\int_{0}^{\infty} u^{2} H(u) \mathrm{d} u \\
& =\lambda_{2} .
\end{aligned}
$$


The final entropy production equation reads

$$
\begin{aligned}
\Sigma=\sum_{i}\left\{\left[\frac{3 \lambda_{2} \phi_{i} \gamma_{i} \mathcal{P}_{i}}{\lambda_{3} \mathcal{R}_{i}^{2}}-\gamma_{I} \frac{\partial \alpha}{\partial \mathcal{R}_{i}}\right] C\left(\mathcal{R}_{i}\right)-\right. & {\left.\left[\frac{3 \lambda_{2} \phi_{i} \gamma_{i} \mathcal{P}_{i}}{\lambda_{3} \mathcal{R}_{i}^{2}}-\gamma_{I} \frac{\partial \alpha}{\partial \mathcal{R}_{i}}\right] D\left(\mathcal{R}_{i}\right)\right\}-\gamma_{I} \frac{\partial \alpha}{\partial r} \frac{\partial r}{\partial t} } \\
& -\left(\gamma_{I} \frac{\partial \alpha}{\partial \phi_{1}}+\Delta \Pi\right) \frac{\tilde{D} \phi_{1}}{\tilde{D} t}+\psi^{*},
\end{aligned}
$$

and making use again of equation (B11) can be reduced to

$$
\Sigma=\sum_{i}\left[\frac{3 \lambda_{2} \phi_{i} \gamma_{i} \mathcal{P}_{i}}{\lambda_{3} \mathcal{R}_{i}^{2}}-\gamma_{I} \frac{\partial \alpha}{\partial \mathcal{R}_{i}}\right] \frac{\mathrm{d} \mathcal{R}_{i}}{\mathrm{~d} t}-\gamma_{I} \frac{\partial \alpha}{\partial r} \frac{\partial r}{\partial t}-\left(\gamma_{I} \frac{\partial \alpha}{\partial \phi_{1}}+\Delta \Pi\right) \frac{\tilde{D} \phi_{1}}{\tilde{D} t}+\psi^{*}
$$

\section{Appendix D: Flow Mechanisms, Viscosities, and Deformation Work Rate}

\section{D1. Micromechanical Deformation Mechanisms}

Even if numerous microscopic, elementary processes are simultaneously involved in ductile rock deformation (dislocation glide and climb, diffusion through grain boundaries or interiors, and grain boundary sliding), the macroscopic deformation is generally described by two main mechanisms to which experimental flow laws are associated:

1. dislocation or grain size insensitive creep

2. diffusion or grain-size sensitive creep

D1.1. Dislocation creep

Dislocation creep requires creation and motion of dislocations in the crystal lattice. Recrystallization operates in parallel to decrease the stored strain energy, resulting, through grain boundaries migration or the formation of subgrains, in creating new, strain-free, grains of smaller size (see; Drury \& Urai, 1990; Shimizu, 2008; Twiss, 1977 for instance). From experimental data, an empirical rheological law as been proposed:

$$
\dot{e}=a_{i} \tau^{n_{i}} \quad \text { Dislocation flow law }
$$

where $\dot{e}$ is the strain rate, $\tau$ is the deviatoric stress, and $a_{i}$ and $n$ are, respectively, a rheological coefficient and an exponent depending on phase $i$ and experimentally calibrated.

\section{D1.2. Diffusion creep}

Diffusion creep mechanism results from atom diffusion through vacancies in the lattice from the regions undergoing maximum stress to the minimum stress regions. The grain atoms could diffuse at grain boundaries (Coble creep, Coble, 1963) or through the grain lattice (Nabarro-Herring creep, Herring, 1950) depending on the intensive conditions of deformation. It could also imply a component of grain boundary sliding. Diffusion creep flow law reads

$$
\dot{e}=b_{i} \tau^{n_{i}} \mathcal{R}_{i}^{-m_{i}} \quad \text { Diffusion flow law }
$$

where $\mathcal{R}$ is a characteristic grain size. The rheological coefficient $b_{i}$ and the exponent $n_{i}$ and $m_{i}$ both depend on the mineral phase $i$ considered. Thus, diffusion creep involves a dependence on grain size. In these two different flow laws, the rheological coefficients $a_{i}$ and $b_{i}$ usually take the form of Arrhenius-type equations:

$$
a_{i}=\mathfrak{a}_{i} \exp \left(\frac{-\mathrm{E}_{i}^{\mathrm{dis}}}{R T}\right) \quad b_{i}=\mathfrak{b}_{i} \exp \left(\frac{-\mathrm{E}_{i}^{\mathrm{diff}}}{R T}\right),
$$

with $\mathfrak{a}_{i}$ and $\mathfrak{b}_{i}$ being prefactors, $E_{i}^{\text {disl }}$ and $E_{i}^{\text {diff }}$ activation energies, $T$ the temperature, and $R$ the gas constant. The prefactors $\mathfrak{a}_{i}, \mathfrak{b}_{i}$, the activation energies $\mathrm{E}_{i}^{\text {disl }}$ and $\mathrm{E}_{i}^{\mathrm{diff}}$, and the exponents $n_{i}, m_{i}$ are constrained by experimental studies. Consequently, they are theoretically only valid for a given mono-phase aggregate with specific chemical composition and grain-sizes distributions. These flow laws defines two mechanical 
domains according to the mean grain size of the deforming aggregate. The transition between these two mechanisms could lead to mechanical weakening provided that the mean grain size could still decrease while the material is deforming in diffusion creep domain.

\section{D2. Effectives Viscosities and Deformational Work Rates}

We consider the aggregate mechanical strength to be controlled by the dislocation and diffusion creep rheologies. The corresponding rheological laws (equation (D1) and (D2)) are assumed equivalent to

$$
\begin{aligned}
& \underline{\tau}_{i}=2 \mu_{i}^{\mathrm{disl}} \underline{\underline{e}}^{\mathrm{disl}}, \\
& \underline{\tau}_{i}=2 \mu_{i}^{\mathrm{diff}} \underline{\underline{e}}^{\mathrm{diff}},
\end{aligned}
$$

where $\tau_{i}$ is the stress tensor, and $\underline{\underline{e}}$ is the strain-rate tensor. These relations are used to define effective viscosities associated with each deformation domain:

$$
\begin{gathered}
\mu_{i}^{\text {disl }}=\frac{1}{2}\left(a_{i} \tau_{i}^{n_{i}-1}\right)^{-1}, \\
\mu_{i}^{\text {diff }}=\frac{1}{2}\left(\frac{\mathcal{R}^{m_{i}}}{b_{i}}\right),
\end{gathered}
$$

with $\tau_{i}$ being the second invariant of the stress tensor in phase $i$. The deformational work rates associated with each mechanism thus yield

$$
\begin{gathered}
\psi_{i}^{\text {diff }}=\underline{\tau}_{i} \vdots \underline{\dot{e}}^{\text {disl }}=2 \mu_{i}^{\text {disl }} \underline{e}^{\text {disl }}: \underline{\dot{e}}^{\text {disl }}=\frac{\dot{e}^{2}}{a_{i} \tau_{i}^{n_{i}-1}}, \\
\psi_{i}^{\text {disl }}=\underline{\tau}_{i}: \underline{\dot{e}}^{\text {diff }}=2 \mu_{i}^{\text {diff } \underline{e}^{\text {diff }}}: \underline{\dot{e}}^{\text {diff }}=\frac{\dot{e}^{2} \mathcal{R}^{m_{i}}}{b_{i}} .
\end{gathered}
$$

We assume that within a phase $i$, all grains share a stress $\underline{\breve{\tau}}_{i}$ equivalent to the macroscopic stress $\tau^{*}$ (Bercovici \& Ricard, 2012, appendix F; Rozel et al., 2011). Furthermore, the deformation of a grain can be due to the two mechanisms acting simultaneously; thus, the total strain rate is assumed to be the sum of the strain rate related to each deformation mechanism. We therefore use a composite rheology of the form:

$$
\underline{\dot{e}}=\underline{\dot{e}}_{i}^{\mathrm{disl}}+\underline{\dot{e}}_{i}^{\mathrm{diff}}=\left(a_{i} \tau_{i}^{n_{i}-1}+\frac{\mathrm{B}_{i}}{\mathcal{R}_{i}^{m_{i}}}\right) \underline{\boldsymbol{\tau}}_{i},
$$

where the coefficient $\mathrm{B}=b_{i} \frac{\lambda_{3-m}}{\lambda_{3}}$ accounts for the effect of grain-size distribution variance on the overall rheology (Rozel et al., 2011). This relation defines the phase $i$ global viscosity:

$$
\mu_{i}=\frac{1}{2}\left(a_{i} \tau_{i}^{n_{i}-1}+\frac{\mathrm{B}_{i}}{\mathcal{R}_{i}^{m_{i}}}\right)^{-1},
$$

and yields the deformational work rate related to each phase

$$
\psi_{i}=\underline{\tau}_{i}: \underline{\dot{e}}=2 \mu_{i} \dot{e}^{2}=\left(a_{i} \tau_{i}^{n_{i}-1}+\frac{\mathrm{B}_{i}}{\mathcal{R}_{i}^{m_{i}}}\right)^{-1} \dot{e}^{2} .
$$

The point where each mechanisms contribute equally to the grain deformation defines a transition grain size $\mathcal{R}_{c i}$ given by the equation:

$$
\mathcal{R}_{c i}=\left(\frac{b_{i}}{a_{i} \tau_{i}^{n_{i}-1}}\right)^{1 / m_{i}}
$$




\section{Appendix E: Table of Used Symbols}

These tables gather all the symbols used in the model description as well as the section number in which they first appear of are defined and the related equation number.

Table E1

Model Parameters

\begin{tabular}{|c|c|c|c|c|}
\hline Symbols & Value & Dimension SI & Description & Reference \\
\hline$T$ & 1,000 & $\mathrm{~K}$ & Temperature & \\
\hline$\gamma$ & 1 & $\mathrm{~J}$ & Surface tension of grain boundaries and interfaces & Duyster and Stockhert (2001) \\
\hline$A_{o l}^{\mathrm{disl}}$ & $1.1010^{-16}$ & $\mathrm{~Pa}^{n_{o l}} \mathrm{~s}^{-1}$ & Prefactor of olivine dislocation creep law & Hirth and Kolhstedt (2003) \\
\hline$E_{o l}^{\text {disl }}$ & $5.3010^{5}$ & $\mathrm{~J} \cdot \mathrm{mol}^{-1} \cdot \mathrm{K}^{-1}$ & Activation energy of olivine dislocation creep law & Hirth and Kolhstedt (2003) \\
\hline$n_{o l}$ & 3.5 & $\varnothing$ & Stress exponent of olivine dislocation creep law & Hirth and Kolhstedt (2003) \\
\hline$A_{o l}^{\mathrm{diff}}$ & $1.5010^{-15}$ & $\mathrm{~m}^{m_{o l}} \cdot \mathrm{Pa}^{-1} \cdot \mathrm{s}^{-1}$ & Prefactor of olivine diffusion creep law & Hirth and Kolhstedt (2003) \\
\hline$E_{o l}^{\text {diff }}$ & $3.7510^{5}$ & $\mathrm{~J} \cdot \mathrm{mol}^{-1} \cdot \mathrm{K}^{-1}$ & Activation energy of olivine diffusion creep law & Hirth and Kolhstedt (2003) \\
\hline$m_{o l}$ & 3 & $\varnothing$ & Grain-size exponent of olivine diffusion creep law & Hirth and Kolhstedt (2003) \\
\hline$A_{A n}^{\mathrm{disl}}$ & $5.0110^{-6}$ & $\mathrm{~Pa}^{n_{\text {dio }}} \mathrm{s}^{-1}$ & Prefactor of diopside dislocation creep law & Rybacki and Dresen (2000) \\
\hline$E_{A n}^{\text {disl }}$ & $6.4810^{5}$ & $\mathrm{~J} \cdot \mathrm{mol}^{-1} \cdot \mathrm{K}^{-1}$ & Activation energy of diopside dislocation creep law & Rybacki and Dresen (2000) \\
\hline$n_{A n}$ & 3 & $\varnothing$ & Stress exponent of diopside dislocation creep law & Rybacki and Dresen (2000) \\
\hline$A_{A n}^{\text {diff }}$ & $1.2610^{-12}$ & $\mathrm{~m}^{m_{\text {dio }}} \cdot \mathrm{Pa}^{-1} \cdot \mathrm{s}^{-1}$ & Prefactor of diospside diffusion creep law & Rybacki and Dresen (2000) \\
\hline$E_{A n}^{\text {diff }}$ & $4.6710^{5}$ & $\mathrm{~J} \cdot \mathrm{mol}^{-1} \cdot \mathrm{K}^{-1}$ & Activation energy of diopside diffusion creep law & Rybacki and Dresen (2000) \\
\hline$m_{A n}$ & 3 & $\varnothing$ & Grain-size exponent of diopside diffusion creep law & Rybacki and Dresen (2000) \\
\hline$A_{\text {dio }}^{\text {disl }}$ & $3.1010^{-28}$ & $\mathrm{~Pa}^{n_{\text {dio }}} \mathrm{s}^{-1}$ & Prefactor of diopside dislocation creep law & Dimanov and Dresen (2005) \\
\hline$E_{d i o}^{\text {disl }}$ & $6.9110^{5}$ & $\mathrm{~J} \cdot \mathrm{mol}^{-1} \cdot \mathrm{K}^{-1}$ & Activation energy of diopside dislocation creep law & Dimanov and Dresen (2005) \\
\hline$n_{\text {dio }}$ & 5.5 & $\varnothing$ & Stress exponent of diopside dislocation creep law & Dimanov and Dresen (2005) \\
\hline$A_{\text {dio }}^{\text {diff }}$ & $3.1910^{-11}$ & $\mathrm{~m}^{m_{\text {dio }} \cdot \mathrm{Pa}^{-1} \cdot \mathrm{s}^{-1}}$ & Prefactor of diospside diffusion creep law & Dimanov and Dresen (2005) \\
\hline$E_{\text {dio }}^{\text {diff }}$ & $5.2810^{5}$ & $\mathrm{~J} \cdot \mathrm{mol}^{-1} \cdot \mathrm{K}^{-1}$ & Activation energy of diopside diffusion creep law & Dimanov and Dresen (2005) \\
\hline$m_{\text {dio }}$ & 3 & $\varnothing$ & Grain-size exponent of diopside diffusion creep law & Dimanov and Dresen (2005) \\
\hline$g_{o l}^{\text {grow }}$ & $2.8010^{-8}$ & $\mathrm{~m}^{p} \mathrm{~s}^{-1}$ & Prefactor of olivine grain-growth law & Karato (1989) \\
\hline$E_{o l}^{\text {grow }}$ & $3.0010^{5}$ & $\mathrm{~J} \cdot \mathrm{mol}^{-1} \cdot \mathrm{K}^{-1}$ & Activation energy of olivine grain-growth law & Karato (1989) \\
\hline$g_{A n}^{\text {grow }}$ & $2.5910^{-4}$ & $\mathrm{~m}^{p} \mathrm{~s}^{-1}$ & Prefactor of anorthite grain-growth law & Dresen et al. (1995) \\
\hline$E_{A n}^{\text {grow }}$ & $3.6510^{5}$ & $\mathrm{~J} \cdot \mathrm{mol}^{-1} \cdot \mathrm{K}^{-1}$ & Activation energy of anorthite grain-growth law & Dresen et al. (1995) \\
\hline$g_{\text {Ens }}^{\text {grow }}$ & $3.3010^{-3}$ & $\mathrm{~m}^{p} \mathrm{~s}^{-1}$ & Prefactor of enstatite grain-growth law & Fisler et al. (1997) \\
\hline$E_{\text {Ens }}^{\text {grow }}$ & $4.0010^{5}$ & $\mathrm{~J} \cdot \mathrm{mol}^{-1} \cdot \mathrm{K}^{-1}$ & Activation energy of enstatite grain-growth law & Fisler et al. (1997) \\
\hline
\end{tabular}

Table E2

Values of the Partition Function Related to Dislocation Creep $\mathfrak{f}_{D i}$

\begin{tabular}{lccc}
\hline Mineral phase & Estimated $\sigma$ & $\mathfrak{f}_{D i}$ with estimated $\sigma$ & $\mathfrak{f}_{D i}$ with $\sigma=0.8$ \\
\hline Olivine & 0.8 & $2.432610^{-8}$ & $2.432610^{-8}$ \\
Plagioclase & 0.8 & $9.844510^{-6}$ & $9.844510^{-6}$ \\
Pyroxene & 0.4 & $5.253110^{-5}$ & $3.814610^{-5}$ \\
\hline
\end{tabular}


Table E3

List of Used Symbols

\begin{tabular}{|c|c|c|c|c|}
\hline Symbol & Description & Dimension SI & Section & Equation no. \\
\hline$\theta_{i}$ & Variable related to one specific phase $(i=1,2)$ & & 2.1 & \\
\hline$\breve{\theta}$ & Variable related to one specific grain & & 2.1 & \\
\hline$\tilde{\theta}$ & Variable related to the interface between phases & & 2.1 & \\
\hline$\theta_{s}$ & Dimension analysis scale & & 4.1 & \\
\hline$\theta^{\prime}$ & Dimensionless variable & $\varnothing$ & 4.1 & \\
\hline $\bar{\theta}$ & Simple weighted average of the variable $\theta, \bar{\theta}=\sum_{i} \phi_{i} \theta_{i}$ & & 2.1 & \\
\hline$\theta^{*}$ & Variable averaged over the entire control volume & & 3.5.3 & (11) \\
\hline$a_{i}$ & Dislocation creep prefactor & $\mathrm{Pa}^{-n} \mathrm{~S}^{-1}$ & $\mathrm{D}$ & (D3) \\
\hline$a_{s i}$ & Scale of dislocation creep prefactor & $\mathrm{Pa}^{-n} \mathrm{~s}^{-1}$ & 4.1 & $(126)$ \\
\hline $\mathfrak{a}_{i}$ & Prefactor of the dislocation creep Arrhenius coefficient & $\mathrm{Pa}^{-n} \mathrm{~s}^{-1}$ & $\mathrm{D}$ & (D3) \\
\hline$A$ & Area & $\mathrm{m}^{2}$ & 2.1 & (3) \\
\hline$\breve{\mathcal{A}}_{i}$ & Effective grain boundary area & $\mathrm{m}^{2}$ & 2.1 & (3) \\
\hline$b_{i}$ & Diffusion creep prefactor & $\mathrm{m}^{m} \cdot \mathrm{Pa}^{-1} \cdot \mathrm{s}^{-1}$ & $\mathrm{D}$ & (D3) \\
\hline $\mathfrak{b}_{i}$ & Prefactor of the diffusion creep Arrhenius coefficient & $\mathrm{m}^{m} \cdot \mathrm{Pa}^{-1} \cdot \mathrm{s}^{-1}$ & $\mathrm{D}$ & (D3) \\
\hline$B$ & Amplitude coefficient of the self-similar distribution function & $\varnothing$ & B1 & (B4) \\
\hline$B_{s i}$ & Scale of corrected diffusion creep prefactor & $\mathrm{m}^{m} \cdot \mathrm{Pa}^{-1} \cdot \mathrm{s}^{-1}$ & 4.1 & (127) \\
\hline $\mathrm{B}_{i}$ & Diffusion creep coefficient variance corrected & $\mathrm{m}^{m} \cdot \mathrm{Pa}^{-1} \cdot \mathrm{s}^{-1}$ & 4.1 & $(131)$ \\
\hline$c$ & Drag coefficient & $\mathrm{s}^{-1}$ & $\mathrm{C} 1$ & (C4) \\
\hline$C\left(\mathcal{R}_{i}\right)$ & Amplitude function accounting for continuous grain-size fluxes & $\mathrm{m} / \mathrm{s}$ & $3.1, \mathrm{~B} 1$ & $(12),(\mathrm{B} 11)$ \\
\hline$C_{i}$ & Coarsening coefficient of phase $i$ & $\varnothing$ & 4.1 & (134) \\
\hline$C_{I}$ & Coarsening coefficient of interface & $\varnothing$ & 4.1 & $(139)$ \\
\hline$D\left(\mathcal{R}_{i}\right)$ & Amplitude function accounting for discontinuous grain-size fluxes & $\mathrm{m} / \mathrm{s}$ & $3.1, \mathrm{~B} 1$ & $(12),(\mathrm{B} 11)$ \\
\hline $\mathrm{D}_{i}$ & Damage coefficient of phase $i$ & $\varnothing$ & 4.1 & (136) \\
\hline $\mathrm{D}_{I}$ & Damage coefficient of interface & $\varnothing$ & 4.1 & $(140)$ \\
\hline$\dot{e}$ & Total strain rate & $\mathrm{s}^{-1}$ & 3.5 .3 & $(118)$ \\
\hline $\mathrm{E}_{i}^{\mathrm{disl}}$ & Dislocation creep activation energy of phase $i$ & $\mathrm{Jmol}^{-1} \mathrm{~K}^{-1}$ & $\mathrm{D}$ & (D3) \\
\hline $\mathrm{E}_{i}^{\mathrm{diff}}$ & Diffusion creep activation energy of phase $i$ & $\mathrm{Jmol}^{-1} \mathrm{~K}^{-1}$ & $\mathrm{D}$ & (D3) \\
\hline $\mathrm{E}_{i}^{\text {grow }}$ & Grain growth activation energy of phase $i$ & $\mathrm{Jmol}^{-1} \mathrm{~K}^{-1}$ & $\mathrm{D}$ & (D3) \\
\hline $\mathbf{f}$ & Vector of thermodynamical forces & & 3.2 & (26) \\
\hline $\mathfrak{f}_{D i}$ & $\begin{array}{l}\text { Partition fraction of the deformational work rate } \\
\text { related to dislocation creep }\end{array}$ & $\varnothing$ & 3.5.1 & (79) \\
\hline $\mathfrak{f}_{N i}$ & $\begin{array}{l}\text { Partition function of the deformational work rate } \\
\text { related to grain damage in the diffusion creep domain }\end{array}$ & $\varnothing$ & 3.5 .1 & $(79)$ \\
\hline $\mathfrak{f}_{\alpha i}$ & Partition fraction of the deformation work rate & $\varnothing$ & 3.5 .2 & $(110)$ \\
\hline $\mathrm{F}$ & Deformation mechanisms partition function & $\varnothing$ & 3.5 .1 & $(79)$ \\
\hline $\mathcal{F}$ & Fineness of the interface (Bercovici \& Ricard, 2012) & $\mathrm{m}^{-1}$ & 2.2 & (7) \\
\hline$g_{i}$ & Prefactor of the grain growth Arrhenius coefficient & $\mathrm{m}^{p} \mathrm{~s}^{-1}$ & 4.2.2 & $(148)$ \\
\hline$G_{i}$ & Grain growth rate of phase $i$ & $\mathrm{~m}^{p} \mathrm{~s}^{-1}$ & 4.2.2 & (148) \\
\hline$G_{I}$ & $\begin{array}{l}\text { Bercovici and Ricard (2013) coarsening rate of mean } \\
\text { interface curvature radius } r\end{array}$ & $\mathrm{~m}^{q} \mathrm{~s}^{-1}$ & 4.2.2 & $(145)$ \\
\hline$\hat{G}_{I}$ & Generic grain growth rate of interface roughness $r$ & $\mathrm{~m}^{q} \mathrm{~s}^{-1}$ & 4.2 .2 & $(146)$ \\
\hline$H$ & Shape function of the self similar distribution & $\varnothing$ & $3.1, \mathrm{~B} 2$ & $(11),(\mathrm{B} 12)$ \\
\hline$i$ & Phase indice $=1$ or 2 & $\varnothing$ & 2.1 & \\
\hline$I_{i}$ & Integrand constant related to phase $i$ & & 3.4 .2 & $(66)$ \\
\hline
\end{tabular}




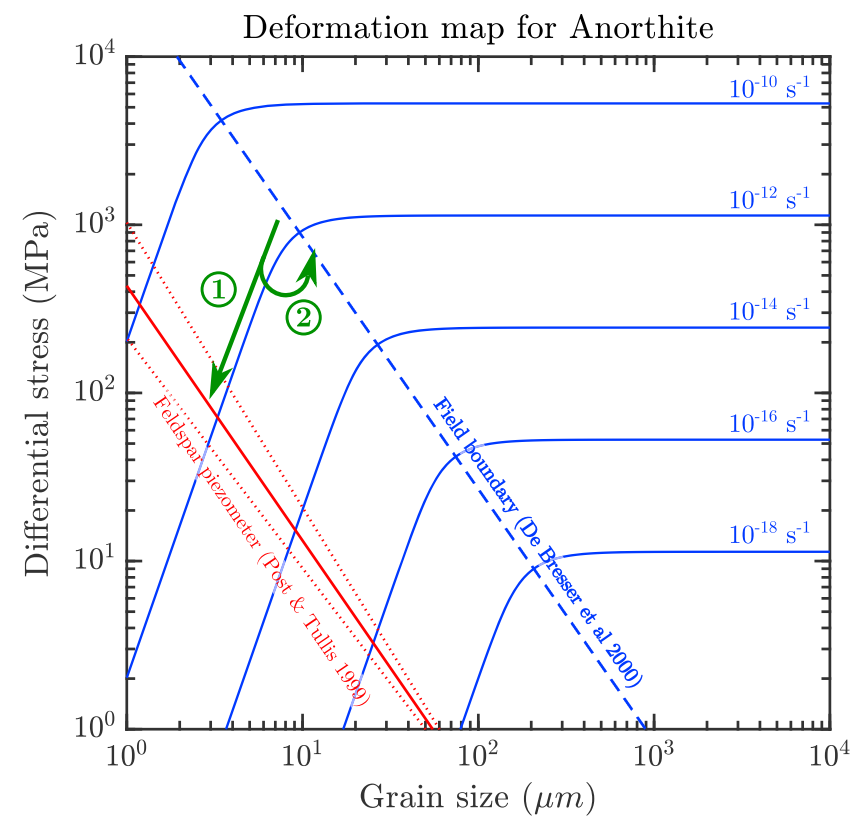

Figure E1. Deformation map for anorthite (blue lines) using Rybacki and Dresen (2000) flow law parameters and computed at 1,000 K. The red line displays the piezometer proposed by Post and Tullis (1999) with the uncertainties (dotted red lines). The dotted blue line corresponds to the fields boundary. As demonstrated by De Bresser et al. (2000), the known behavior of grains deforming under dislocation and diffusion creep alone implies a steady-state grain size limited to the boundary between the two domains (green arrows 1). In order to reach the piezometric grain size, other mechanisms are needed (green arrows 2).
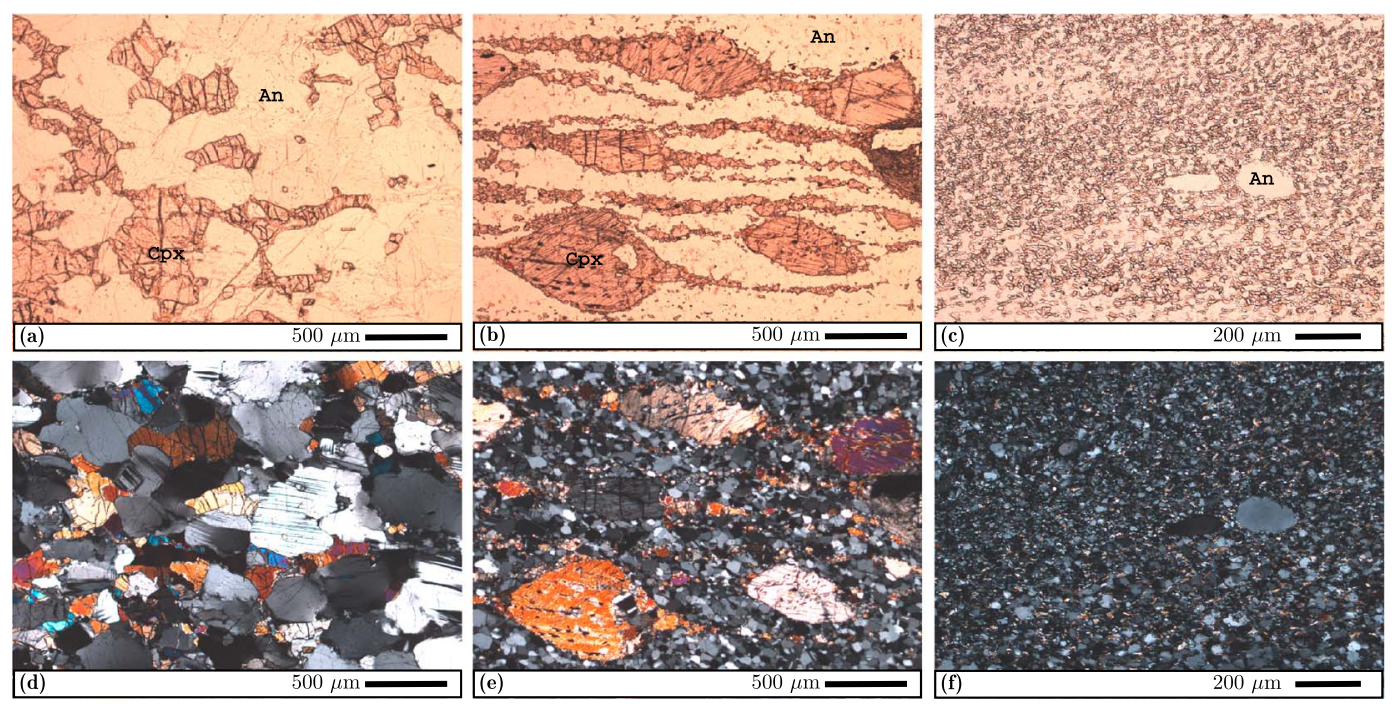

Figure E2. Optical microscope images of thin sections presenting different textures exhibited by the Hidaka shear zone (Hokkaido, Japan). Upper pictures are in plane light and lower ones in cross-polarized light. (a) and (d) present the quasi-undeformed texture. (b) and (e) display a classical dislocation creep texture where pyroxenes grains tend to cluster around porphyroclasts and plagioclase ones form layers. Grain sizes are decreasing through predominant dynamic recrystallization. (c) and (f) illustrate a pattern of very fine and well-mixed grain where grain boundary sliding-induced nucleation is suspected. All the samples consist of mylonitized gabbro deforming under granulitic/amphibolitic conditions. 
Table E4

List of Used Symbols

\begin{tabular}{|c|c|c|c|c|}
\hline Symbol & Description & Dimension SI & Section & Equation no. \\
\hline $\mathbf{J}$ & Vector of thermodynamical fluxes & & 3.2 & (18) \\
\hline$k$ & Indice of the phenomenological coefficients & & 3.2 & (18) \\
\hline$\underline{\mathbf{K}}$ & Matrix of phenomenological coefficients & & 3.2 & (18) \\
\hline$K_{k k}$ & Phenomenological coefficient & & 3.2 & (18) \\
\hline $\mathrm{K}$ & Mean curvature of a grain surface & $\mathrm{m}^{-1}$ & 2.1 & (4) \\
\hline $\mathcal{K}$ & Mean curvature of a grain boundaries averaged over the volume $\Delta V$ & $\mathrm{~m}^{-1}$ & 2.1 & (4) \\
\hline$l$ & Indice of the phenomenological coefficients & & 3.2 & (18) \\
\hline$m$ & Grain-size exponent of the diffusion creep law & $\varnothing$ & 3.5 .3 & $(118)$ \\
\hline$\breve{\mathrm{m}}_{i}$ & Mass of one grain of size $\breve{\mathrm{R}}_{i}$ & $\mathrm{~kg}$ & A & (A15) \\
\hline $\mathrm{M}_{i}$ & Total mass of phase $i$ & $\mathrm{~kg}$ & A & (A15) \\
\hline$n$ & Stress exponent of the dislocation creep law & $\varnothing$ & 3.5.3 & (117) \\
\hline $\mathrm{n}_{i}$ & Number of grain of phase $i$ & $\varnothing$ & A & (A3) \\
\hline$\hat{\mathbf{n}}$ & Unit vector normal to element $\mathrm{d} A$ & $\varnothing$ & A & (A6) \\
\hline$P$ & Pressure & $\mathrm{Pa}$ & $\mathrm{C} 1$ & $(\mathrm{C} 2)$ \\
\hline $\mathrm{P}$ & Roughness variable relative to one grain & $\varnothing$ & 2.1 & (3) \\
\hline $\mathcal{P}$ & Mean grain roughness variable & $\varnothing$ & 2.1 & \\
\hline$q$ & Heat fluxes across the control volume surface & $\mathrm{J} / \mathrm{m}^{2}$ & $\mathrm{C} 1$ & $(\mathrm{C} 1)$ \\
\hline$Q$ & Heat source within the control volume & $\mathrm{J}$ & $\mathrm{C} 1$ & (C1) \\
\hline$r$ & $\begin{array}{l}\text { Interface mean curvature radius of curvature } \\
\text { (Bercovici \& Ricard, 2012) }\end{array}$ & $\mathrm{m}$ & 2.2 & $(10)$ \\
\hline$R$ & Gas constant & $\mathrm{J} \cdot \mathrm{mol}^{-1} \cdot \mathrm{K}^{-1}$ & 4.2 .2 & $(148)$ \\
\hline $\mathrm{R}$ & $\begin{array}{l}\text { Grain-size variable in the grain-size space } \\
\text { (radius of the sphere equivalent of a specific grain) }\end{array}$ & $\mathrm{m}$ & 2.1 & (2) \\
\hline $\mathcal{R}$ & Mean grain-size variable & $\mathrm{m}$ & 2.1 & \\
\hline $\mathcal{R}_{c i}$ & Transition grain size between GSI and GSS creep & $\mathrm{m}$ & 3.5 .1 & (84) \\
\hline $\mathcal{R}_{s}$ & Grain-size (microscopical) scale & $\mathrm{m}$ & 4.1 & $(126)$ \\
\hline$S$ & Mixture variable & $\varnothing$ & 3.5.3 & $(115)$ \\
\hline$s$ & Total entropy & $\varnothing$ & 3.5.3 & $(115)$ \\
\hline$\breve{S}_{i}$ & $\begin{array}{l}\text { Entropy of one grain of phase } i \\
\text { between grain size and interface }\end{array}$ & $\varnothing$ & 3.5 .3 & $(115)$ \\
\hline$t$ & Time variable & $\mathrm{s}$ & 4.1 & (124) \\
\hline$t_{s}$ & Time scale & $\mathrm{s}$ & 4.1 & (124) \\
\hline$T$ & Temperature & K & 4.2 .2 & $(148)$ \\
\hline$u$ & Self-similarity variable $u=\mathrm{R} / \mathcal{R}$ & $\varnothing$ & B1 & (B1) \\
\hline$V$ & Volume & $\mathrm{m}^{3}$ & 2.1 & (1) \\
\hline $\mathbf{v}$ & Velocity tensor & $\mathrm{m} / \mathrm{s}$ & 4.1 & \\
\hline$x$ & Exponent of partition function & $\varnothing$ & 3.5 .1 & $(82)$ \\
\hline $\mathbf{x}$ & Vector of space coordinates & $\varnothing$ & B & (B1) \\
\hline$X$ & Undetermined partition function & $\varnothing$ & 3.5 .1 & (77) \\
\hline $\mathrm{X}$ & Vector of dissipative forces & & 3.2 & (17) \\
\hline $\mathbf{Y}$ & Vector of state variables & & 3.2 & (20) \\
\hline$\dot{\mathbf{Y}}$ & Vector of kinetic variables & & 3.2 & $(20)$ \\
\hline $\mathrm{Z}$ & Coefficient of Zener pinning & $\varnothing$ & 4.1 & $(136)$ \\
\hline$z$ & Amplitude coefficient of Zener pinning & $\varnothing$ & 3.4 .2 & $(58)$ \\
\hline
\end{tabular}

Note. GSI = grain size insensitive; GSS = grain size sensitive. 


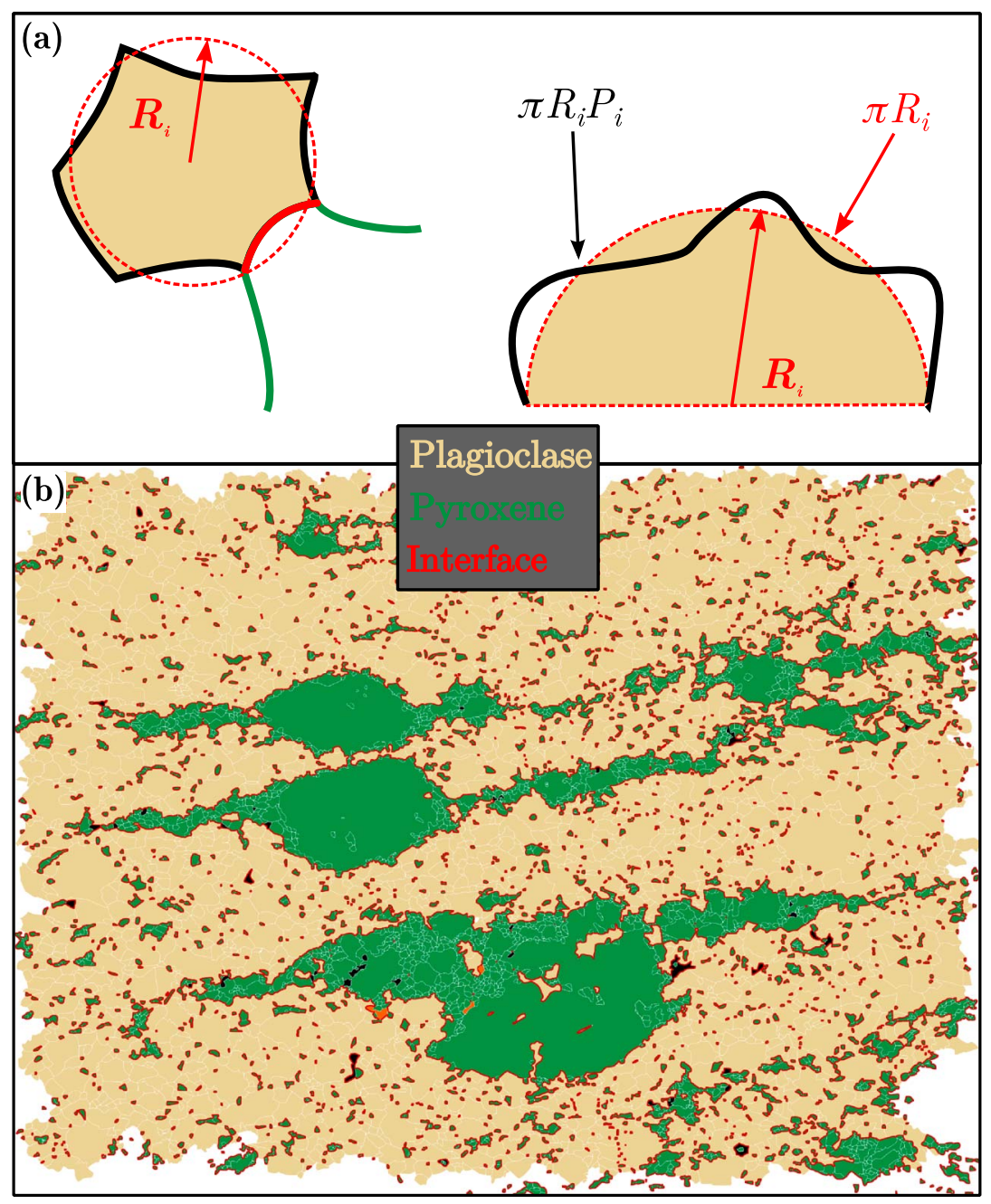

Figure E3. (a) Sketch representing the overall assumptions and relations between the "real" two-phase aggregate and its physical representation in the model. (b) Manually segmented pictures of a quasi-two-phase aggregate (gabbro from Hidaka, Japan), illustrating the relationship between phase proportion, grain-size distributions, and the mean curvature radius of the interface $r$. The mixture quality of the aggregate is a function of all these variables. The orange and black grains are scarce amphiboles and oxides, respectively. 

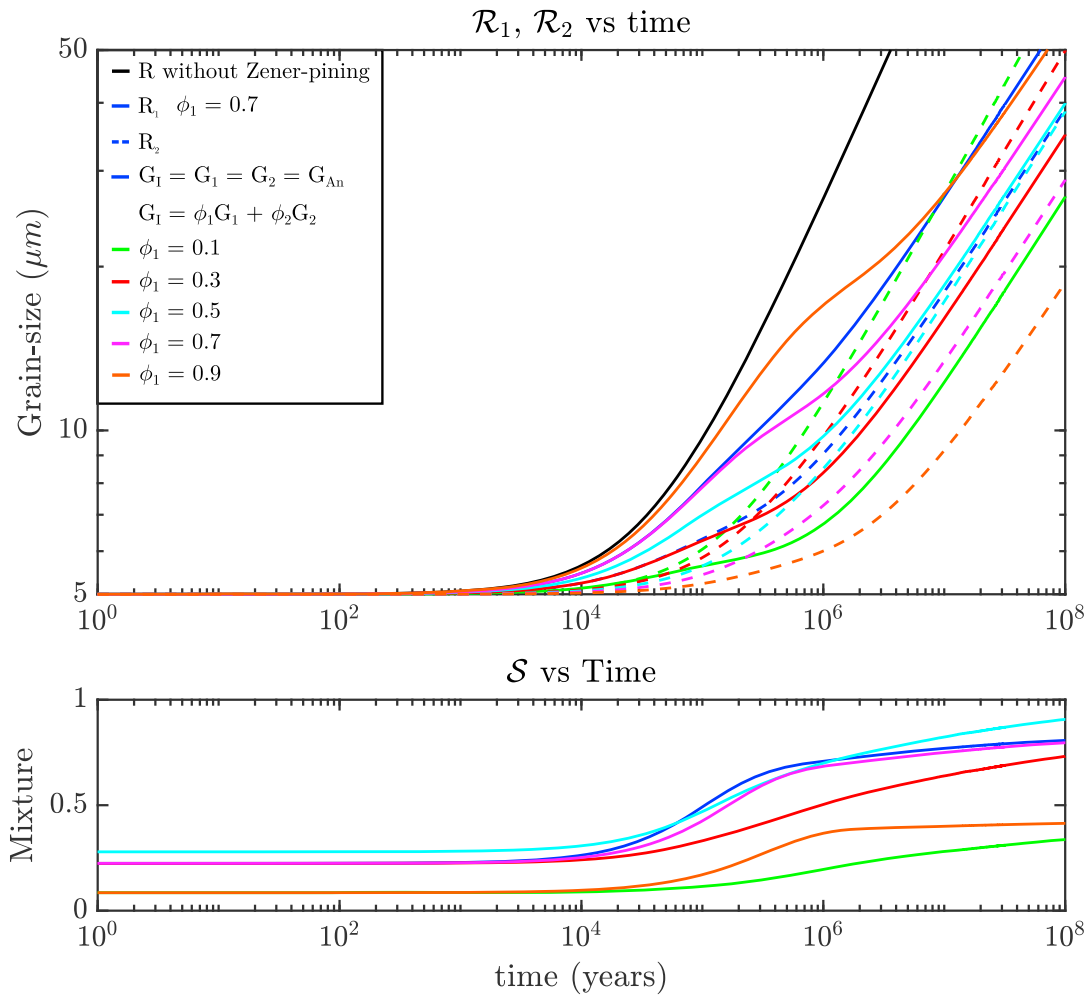

Figure E4. Evolution of the two grain size $\mathcal{R}_{1}, \mathcal{R}_{2}$ without deformation at a temperature of $1,000 \mathrm{~K}$. All initial grain size are set up to $5 \mu \mathrm{m}$. (black) Grain size computed with anorthite grain-growth law (Dresen et al., 1995) without active Zener pinning. (blue curves) The two phases share anorthite grain-growth law (Dresen et al., 1995) with volumic fractions $\phi_{1}=0.7$. (green to brown curves) Grain sizes computed with anorthite grain-growth law (Dresen et al., 1995) for phase 1 and enstatite grain-growth law (Fisler et al., 1997) for phase 2 at varying phase volumic fractions. Using different grain growth laws for the two phase shows little difference in the final grain sizes at $10^{9}$ years, the main controlling parameter being the phase volumic fractions. The maximum mixing state is reached for $\phi_{1}=\phi_{2}=0.5$, while much larger difference in phase proportion decreases the value of the maximum $S$. 
Table E5

List of used symbols

\begin{tabular}{|c|c|c|c|c|}
\hline Symbol & Description & Dimension SI & Section & Equation no \\
\hline$\alpha$ & Interface area volume density & $\mathrm{m}^{-1}$ & 3.4 .2 & (66) \\
\hline$\alpha_{i}$ & Grain boundary volume density & $\mathrm{m}^{-1}$ & 3.1 & (15) \\
\hline$\gamma_{i}$ & Surface tension of intraphase grain boundaries & $\mathrm{s}^{-2}$ & 3.1 & (13) \\
\hline$\gamma_{I}$ & Effective surface tension of interfaces & $\mathrm{s}^{-2}$ & 3.1 & (13) \\
\hline$\Gamma_{i}$ & Discontinuous flux of grain size in grain-size space & $\mathrm{s}^{-1}$ & B1 & (B8) \\
\hline$v_{i}$ & Grain-size distribution function of phase $i$ & $\varnothing$ & $3.1, \mathrm{~B} 1$ & $(11),(\mathrm{B} 1)$ \\
\hline$\Pi_{i}$ & Effective pressure within phase $i, \Pi=P_{i}+\gamma_{i} \mathcal{K}_{i}$ & $\mathrm{~Pa}$ & 3.3 & (39) \\
\hline$\rho_{i}$ & Density of phase $i$ & $\mathrm{~kg} / \mathrm{m}^{3}$ & $\mathrm{C} 1$ & $(\mathrm{C} 2)$ \\
\hline$\lambda_{n}$ & $\begin{array}{l}n \text {th moment of the grain-size distribution } \\
\text { (Rozel et al., 2011) }\end{array}$ & $\varnothing$ & $3.1, \mathrm{~B} 1$ & (14),(B13) \\
\hline$\mu_{i}$ & Effective viscosity of phase $i$ & Pas & 3.5 .3 & (120) \\
\hline$\sigma$ & $\begin{array}{l}\text { Variance of the grain-size distribution } \\
\text { normalized by the mean }\end{array}$ & $\varnothing$ & 3.1 & (14) \\
\hline$\sigma(\mathcal{R})$ & True variance of the grain-size distribution & $\mathrm{m}^{2}$ & B2 & (B15) \\
\hline$\Sigma$ & Entropy production & $\mathrm{J} \cdot \mathrm{K}^{-1} \cdot \mathrm{s}^{-1}$ & 3.1 & (13) \\
\hline$\tau$ & Stress (second invariant of the stress tensor) & $\mathrm{Pa}$ & 3.5 .3 & (117) \\
\hline$\phi_{i}$ & Phase $i$ volume fraction & $\varnothing$ & 2.1 & (1) \\
\hline$\chi_{i}$ & Phase $i$ deformation work rate partition coefficient & $\varnothing$ & 3.5 .2 & (110) \\
\hline$\varphi_{i}$ & Phase $i$ spacial continuous function & $\varnothing$ & 2.1 & (1) \\
\hline$\psi$ & Deformation work rate & W & 3.5 .3 & (122) \\
\hline
\end{tabular}

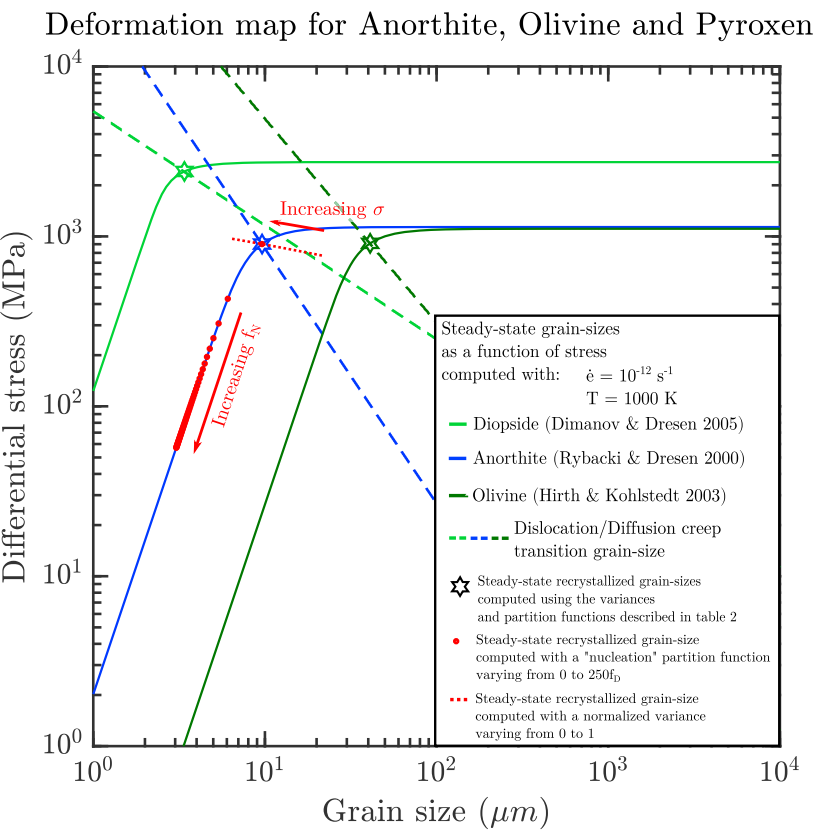

Figure E5. Deformation map for olivine, anorthite, and diopside computed at a strain rate of $10^{-12} \mathrm{~s}^{-1}$. The grain size/stress curves are displayed as solid lines, and the grain size insensitive/grain size sensitive domains are defined by the blue and green dashed lines (the field boundary limit; De Bresser et al., 2000). The stars display the steady-state grain size computed using the partitions functions $\mathfrak{f}_{D}$ and the variances $\sigma$ displayed in Table E2 for each mineral phases. The red solid line presents the effect of a varying partition function $\mathfrak{f}_{N}$ on the steady-state grain size $\left(\mathfrak{f}_{N}\right.$ varying between 0 and $250 \mathfrak{f}_{D}$ ). The dashed red lines illustrates the effect of a varying variance on the steady-state grain size (from 0 to 1). Generally, a higher dimensionless variance $\sigma$ results in a smaller steady-state mean grain size at fixed temperature and strain rate. 
(a)
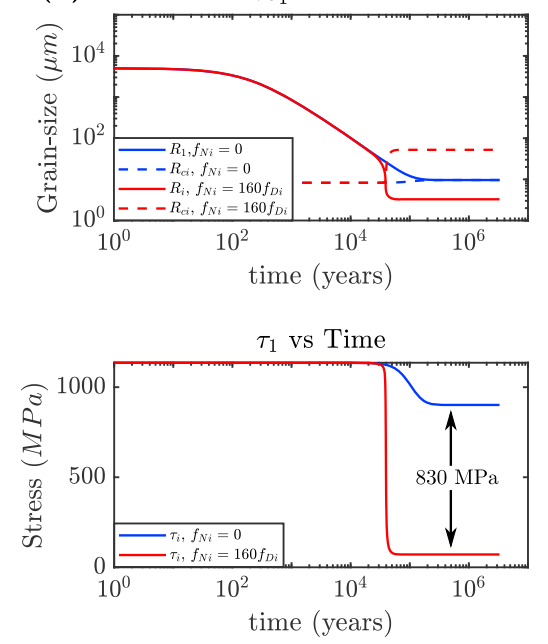

\begin{tabular}{l|l}
\hline Phase rheology = anorthite & $\begin{array}{l}\dot{e}=10^{-12} \mathrm{~s}^{-1} \\
\mathcal{P}_{i}=1.2\end{array}$ \\
Initial $\mathcal{R}_{i}=r=5 \mathrm{~mm}$ &
\end{tabular} (b) Deformation map for Anorthite

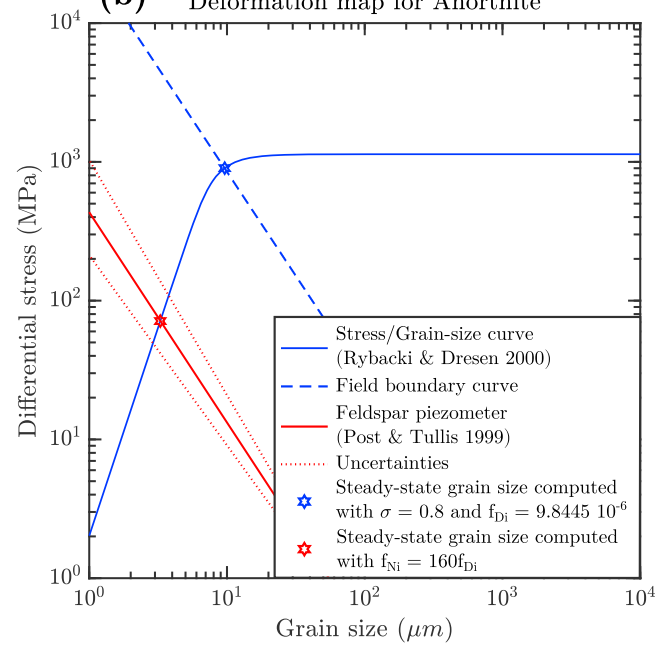

Grain size $(\mu m)$

Figure E6. (a) Evolution of the aggregates variables (mean grain sizes $\mathcal{R}_{i}$, grain size insensitive/grain size sensitive transition grain-sizes $\mathcal{R}_{c i}$, and stresses $\tau_{i}$ during deformation without dissipation of energy in the grain size sensitive domain [blue lines] and when such a mechanism is occurring [red lines]). Comparing the grain-size evolution yields as expected, smaller steady-state grain size and lower stress when deformation energy dissipation is possible in the diffusion creep domain. In this case a partition function $\mathfrak{f}_{N i}$ of about 160 time $\mathfrak{f}_{D i}$ is required to reach the steady-state grain size predicted by the Post and Tullis (1999) piezometer. (b) Deformation map for anorthite comparing the computed steady-state grain sizes with the stress/grain-size curve computed using Rybacki and Dresen (2000) flow laws and the Post and Tullis (1999) piezometer. 
(a)
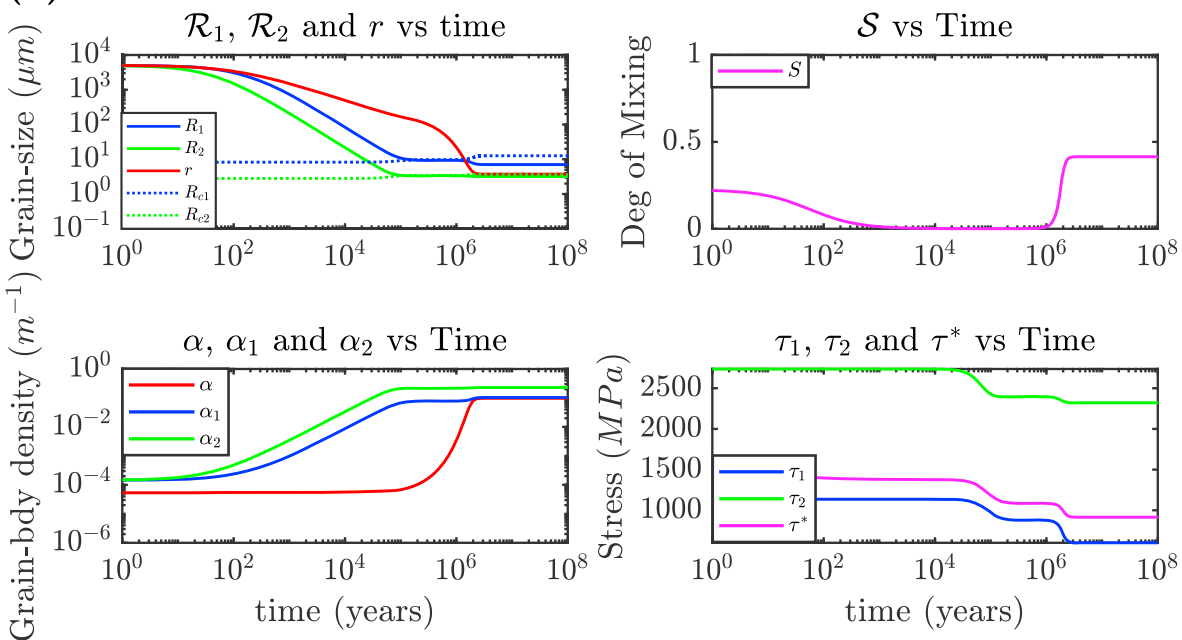

\begin{tabular}{lll}
\hline Phase $1=$ anorthite & $\dot{e}=10^{-12} \mathrm{~s}^{-1}$ & $T=1000 \mathrm{~K}$ \\
Phase $2=$ diopside & $\mathcal{P}_{i}=1.2$ & $\phi_{1}=0.7$ \\
Initial $\mathcal{R}_{i}=r=5 \mathrm{~mm}$ & $\chi_{i}=1$ & $\mathfrak{f}_{D i}=\mathrm{cf}$ Table 2 \\
\hline
\end{tabular}

(b)
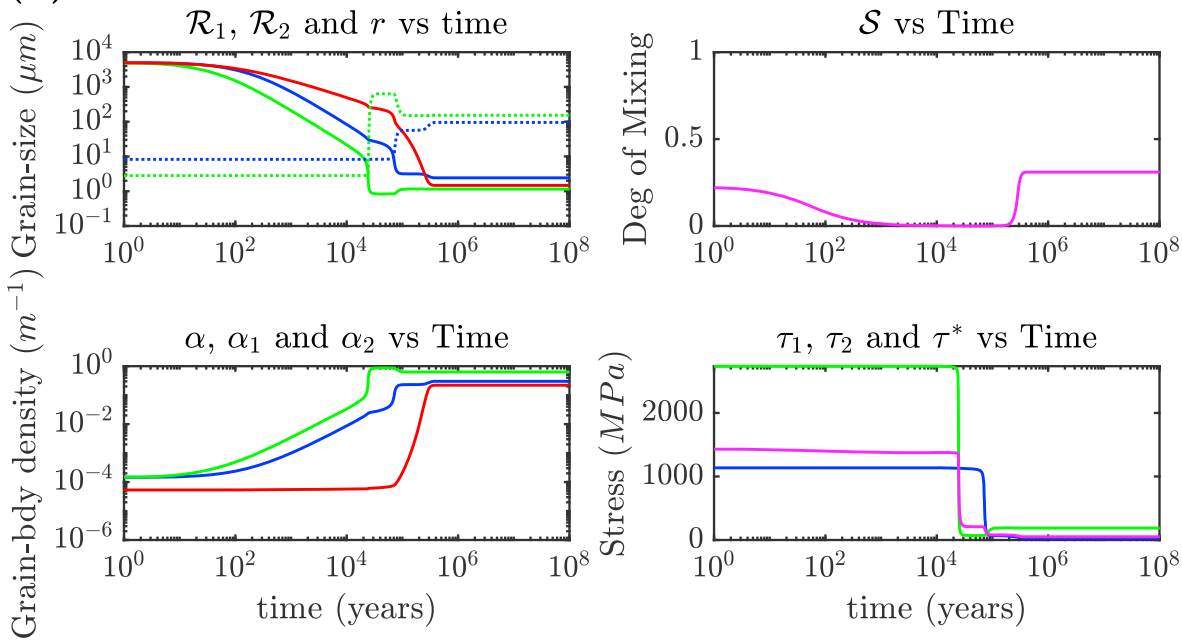

Figure E7. Time evolution of the phases mean grain sizes $\left(\mathcal{R}_{i}\right)$, interface mean radius of curvature $r$, degree of mixing $S$ grain boundary and interface densities $\left(\alpha\right.$ and $\left.\alpha_{i}\right)$, and stresses $\left(\tau_{i}\right)$ describing gabbroic texture evolution during deformation. The rheological parameters are described in Table E1. (a) No storage of deformation work is allowed in the diffusion creep regime. The two phases mean grain size stabilize near the field boundary (dotted lines). The interface mean radius of curvature $r$ decrease slowly during dislocation creep (clusters formation) and more rapidly when diffusion creep becomes dominant. The mixing degree $S$ describes this evolution with a decrease during dynamic recrystallization and a important increase when diffusion creep is active. (b) If deformation energy storage is allowed in the diffusion creep regime, the two phases depict a sharp decrease near the field boundary. The steady-state mean grain size are much smaller, and the resulting stress drop appears very sharp and occurs much earlier than the previous case. 
Deformation map for Anorthite and Diopside

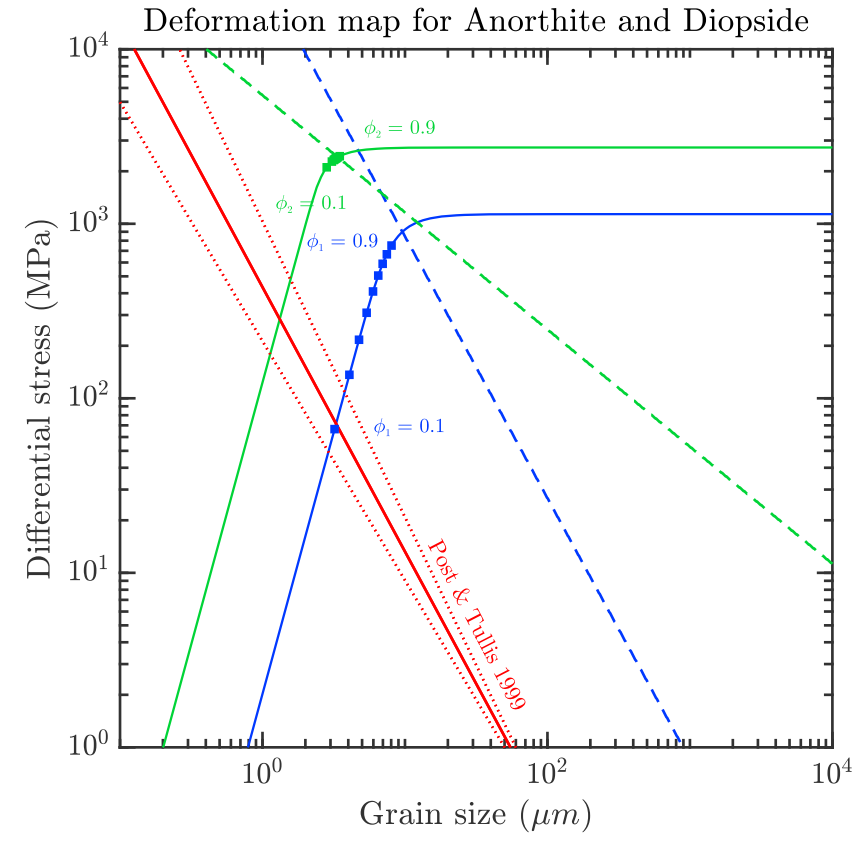

Figure E8. Evolution of a gabbro aggregate during deformation. (a) Time evolution of phases mean grain sizes $\left(\mathcal{R}_{i}\right)$, interface mean radius of curvature $r$, degree of mixing $S$ grain boundary and interface densities $\left(\alpha\right.$ and $\left.\alpha_{i}\right)$, and stresses $\left(\tau_{i}\right)$. Even in the absence of work storage in the diffusion creep domain, the coupling between interface and grain-size evolution allows the two phases to reach smaller steady-state grain sizes. (b) Deformation map computed for anorthite (blue solid line) and diopside (green solid line) along with the Post and Tullis (1999) piezometer for feldspar (red solid lines). The dashed blue and green lines display the computed steady-state grain size for anorthite and diopside, respectively, when we vary the phase volumic fraction from 0.1 to 0.9 .

Acknowledgments

This study was funded by the European Research Council (ERC) under the seventh framework program of the European Union (ERC advanced Grant, grant agreement 290864, RHEOLITH) and by the Institut Universitaire de France. It also benefited from important contributions of the JSPS KAKENHI grant 15K21755 for Benoit Bevillard, Guillaume Richard, and Hikaru Iwamori, the UK Engineering and Physical Sciences Research Council (EPSRC grant EP/K032208/1), the Isaac Newton Institute for the participation of Benoit Bevillard and Guillaume Richard to the Melt in the Mantle Program. The authors deeply thank Jacques Precigout, Hikaru Iwamori, and Thibault Duretz for the valuable discussions that greatly benefited to this study and David Bercovici for the constructive comments made in a previous review. The Matlab script and input files used to produce the figures are available at https://zenodo.org/record/2539301.

\section{References}

Austin, N. J., \& Evans, B. (2007). Paleowattmeters: A scaling relation for dynamically recristallized grain size. Geology, 35, 343-346. Bercovici, D., \& Ricard, Y. (2003). Energetics of a two-phase model of lithospheric damage, shear localization and plate-boundary formation. Geophysical Journal International, 152, 581-596.

Bercovici, D., \& Ricard, Y. (2005). Tectonic plate generation and two-phase damage void growth versus grain size reduction. Journal of Geophysical Research, 110, B03401. https://doi.org/10.1029/2004JB003181

Bercovici, D., \& Ricard, Y. (2012). Mechanisms for the generation of plate tectonics by two phase grain damage and pinning. Physics of the Earth and Planetary Interiors, 202-203, 27-55.

Bercovici, D., \& Ricard, Y. (2013). Generation of plate tectonics with two-phase grain-damage and pinning: Source sink model and toroidal flow. Earth and Planetary Science Letters, 365, 275-288.

Bercovici, D., Ricard, Y., \& Schubert, G. (2001). A two-phase model for compaction and damage 1 general theory. Journal of Geophysical Research, 106(B5), 8887-8906.

Bercovici, D., \& Skemer, P. (2017). Grain damage, phase mixing and plate-boundary formation. Journal of Geodynamics, $108,40-55$.

Brodhag, S. H., \& Herwegh, M. (2010). The effect of different second-phase particle regimes on grain growth in two-phase aggregates: Insights from in situ rock analogue experiments. Contributions to Mineralogy and Petrology, 160, 219-238. https://doi.org/10.1007/s00410-009-0474-6

Carreras, J. (2001). Zooming on Northern Cap de Creus shear zones. Journal of Structural Geology, 23, 1457-1486.

Coble, R. (1963). A model for boundary diffusion controlled creep in polycrystalline materials. Journal of Applied Physics (US), 34, 1679-1682.

Cross, A. J., \& Skemer, P. (2017). Ultramylonite generation via phase mixing in high-strain experiments. Journal of Geophysical Research Solid Earth, 122, 1744-1759. https://doi.org/10.1002/2016JB013801

Czaplinska, D., Piazolo, S., \& Zibra, I. (2015). The influence of phase and grain size distribution on the dynamics of strain localization in polymineralic rocks. Journal of Structural Geology, 72, 15-32.

De Bresser, J. H. P., Ter Heege, J. H., \& Spiers, C. J. (2000). Grain-size reduction by dynamic recrystallisation: Can it result in major rheological weakening? International Journal of Earth Sciences, 90, 28-45.

De Groot, S., \& Mazur, P. (1984). Non-equilibrium thermodynamics. New york: Dover publications.

Derby, B. (1991). The dependence of grain size on stress during dynamic recrystallisation. Acta Metallica Materials, 39(5), 955-962.

Derby, B., \& Ashby, M. F. (1987). On dynamic recrystallisation. Scripta Metallurgica, 21, 879-884.

Dimanov, A., \& Dresen, G. (2005). Rheology of synthetic anorthite-diopside aggregates: Implications for ductile shear zones. Journal of Geophysical Research, 110, B07203. https://doi.org/10.1029/2004JB003431

Dimanov, A., Lavie, M. P., Dresen, G., Ingrin, J., \& Jaoul, O. (2003). Creep of polycrystalline anorthite and diopside. Journal of Geophysical Research, 108(B1), 2061. https://doi.org/10.1029/2002JB001815

Dimanov, A., Ribacki, E., Wirth, R., \& Dresen, G. (2007). Creep and strain-dependent microstructures of synthetic anorthite diopside aggregates. Journal of Structural Geology, 29, 1049-1069. https://doi.org/10.1016/j.jsg.2007.02.010

Dresen, G., Wang, Z., \& Bai, Q. (1995). Kinetics of grain growth in anorthite. Tectonophysics, 258, 251-262. 
Drew, D. A., \& Segel, L. A. (1971). Averaged equation for two-phase flows. Studies in Applied Mathematics, 3, $205-230$.

Drury, M., Lallemant, H. V., Pennock, G., \& Palasse, L. (2011). Crystal preferred orientation in peridotite ultramylonites deformed by grain size sensitive creep, Etang de Lers, Pyrenees, France. Journal of Structural Geology, 33, 1776-1789.

Drury, M. R., \& Urai, J. L. (1990). Deformation-related recrystallization processes. Tectonophysics, 172, $235-253$.

Drury, M. R., Vissers, R. L. M., Van der Wal, D., \& Strating, E. H. H. (1991). Shear localisation in upper mantle peridotites. Pure and Applied Geophysics, 137(4), 439-460.

Duyster, J., \& Stockhert, B. (2001). Grain boundary energies in olivine derived from natural microstructures. Contributions to Mineralogy and Petrology, 104, 567-576.

Faul, U. H., \& Scott, D. (2006). Grain growth in partially molten olivine aggregates. Contributions to Mineralogy and Petrology, 151, 101-111. Feltham, P. (1957). Grain growth in metals. Acta Metallica Materials, 5, 97-105.

Fischer, F. D., Svoboda, J., \& Petryk, H. (2014). Thermodynamic extremal principles for irreversible processes in materials science. Acta Materialia, 67, 1-20.

Fisler, D. K., Mackwell, S. J., \& Petsch, S. (1997). Grain boundary diffusion in enstatite. Physics and Chemistry of Minerals, 24, 264-273.

Fossen, H., \& Cavalcante, G. C. G. (2017). Shear zones-A review. Earth Science Reviews, 171, 434-455. https://doi.org/10.1016/ j.earscirev.2017.05.002

Ganesan, S., \& Poirier, D. R. (1990). Conservation of mass and momentum for the flow of interdendritic liquid during solidification. Metallurgical and Materials Transactions A: Physical Metallurgy and Materials Science, B 21, 173-181.

Hashim, L. (2016). Unraveling the grain size evolution in the Earth's mantle (Ph.D. thesis), UniversitÃl'd'OrlÃl'ans.

Heilbronner, R., \& Barrett, S. (2014). Image analysis in Earth sciences. Berlin, Heidelberg: Springer.

Herring, C. (1950). Diffusional viscosity of a polycrystalline solid. Journal of Applied Physics, 21(5), 437-445.

Herwegh, M., De Bresser, J. H. P., \& Ter Heege, J. H. (2005). Combining natural microstructures with composite flow laws: An improved approach for the extrapolation of lab data to nature. Journal of Structural Geology, 27, 503-521.

Herwegh, M., Linckens, J., Ebert, A., Berger, A., \& Brodhag, S. H. (2011). The role of second phases for controlling microstructural evolution in polymineralic rocks: A review. Journal of Structural Geology, 33, 1728-1750.

Hill, R. (1965). Self-consistent mechanics of composite materials. Journal of Mechanics and Physics of the Solid, 13, 213-222.

Hillert, M. (1965). On the theory of normal and abnormal grain growth. Acta Metallurgica, 13, 227-238.

Hiraga, T., Tachibana, C., Ohashi, N., \& Sano, S. (2010). Grain growth systematics for forsterite +/- enstatite aggregates: Effect of lithology on grain size in the upper mantle. Earth and Planetary Science Letters, 291, 10-20.

Hirth, G., \& Kolhstedt, D. (2003). Rheology of the upper mantle and the mantle wedge: A view from the experimentalists. In J. Eiler (Ed.), Inside the subduction factory (Vol. 138, pp. 83-105). Washington DC: American Geophysical union.

Kameyama, M., Yuen, D. A., \& Fujimoto, H. (1997). Interactions of viscous heating with grain-size dependent rheology in the formation of localized slip zones. Geophysical Research Letters, 24(20), 2523-2526.

Karato, S. I. (1984). Grain size distribution and the rheology of the upper mantle. Tectonophysics, 104, 155-176.

Karato, S. (1989). Grain growth kinetics in olivine aggregates. Tectonophysics, 168, 255-273.

Karato, S. I., Paterson, M. S., \& FitzGerald, J. D. (1986). Rheology of synthetic olivine aggregates: Influence of grain size and water. Journal of Geophysical Research, 91(B8), 8151-8176.

Kaus, B. J. P., \& Podladchikov, Y. Y. (2006). Initiation of localized shear zones in viscoelastoplastic rocks. Journal of Geophysical Research, 111, B04412. https://doi.org/10.1029/2005JB003652

Kohlstedt, D. L. (2007). Properties of rocks and minerals-Constitutive equations, rheological behavior, and viscosity of rocks. In Treatise on geophysics (Vol. 2, pp. 389-417): Elsevier. https://doi.org/10.1016/B978-044452748-6.00043-2

Kohlstedt, D. L., Evans, B., \& Mackwell, S. J. (1995). Strength of the lithosphere: Constraints imposed by laboratory experiments. Journal of Geophysical Research, 100(B9), 17,587-17,602.

Kruse, R., \& Stunitz, H. (1999). Deformation mechanisms and phase distribution in mafic high-temperature mylonites from the Jotun Nappe, Southern Norway. Tectonophysics, 303, 223-249.

Leloup, P. H., Ricard, Y., Battaglia, J., \& Lacassin, R. (1999). Zones heating in continental strike-slip shear zones: Model and field examples. Geophysical Journal International, 136, 19-40.

Linckens, J., Herwegh, M., \& Muntener, O. (2015). Small quantities but large effect-How minor phases control shear localization in upper mantle shear zones. Tectonophysics, 643, 26-43.

Mancktelow, N. S. (2002). Finite-element modelling of shear-zone development in viscoelastic materials and its implications for localisation of partial melting. Journal of Structural Geology, 24, 1045-1053.

Mehl, L., \& Hirth, G. (2008). Plagioclase preferred orientation in layered mylonites: Evaluation of flow laws for the lower crust. Journal of Geophysical Research, 113, B05202. https://doi.org/10.1029/2007JB005075

Menegon, L., Fusseis, F., Stunitz, H., \& Xiao, X. (2015). Creep cavitation bands control porosity and fluid flow in lower crustal shear zones. Geology, 43(3), 227-230. https://doi.org/10.1130/G36307.1

Mulyukova, E., \& Bercovici, D. (2017). Formation of lithospheric shear zones: Effect of temperature on two-phase grain damage. Physics of Earth and Planetary Interiors, 270, 195-212.

Nabarro, F. (1967). Steady-state diffusional creep. Philosophical Magazine, 16, 231-237.

Onsager, L. (1931). Reciprocal relations in irreversible processes. Physical Review, 37, 405.

Passchier, C. W., \& Trouw, R. A. (2006). Microtectonics (2nd ed., Vol. 144): Cambridge University Press. https://doi.org/10.1017/ S0016756806002792

Pennachioni, G., \& Mancktelow, N. S. (2007). Nucleation and initial growth of a shear zone network within compositionally and structurally heterogeneous granitoids under amphibolite facies conditions. Journal of Structural Geology, 29, 1757-1780.

Platt, J. P. (2015). Rheology of two-phase systems: A microphysical and observational approach. Journal of Structural Geology, 77, 213-227.

Poirier, J. P. (1980). Shear localization and shear instability in materials in the ductile field. Journal of Structural Geology, 2(1/2), 135-142.

Post, A., \& Tullis, J. (1999). A recrystallized grain size piezometer for experimentally deformed feldspar aggregates. Tectonophysics, 303, 159-173.

Precigout, J., \& Stunitz, H. (2016). Evidence of phase nucleation during olivine diffusion creep: A new perspective for mantle strain localisation. Earth and Planetary Science Letters, 405, 94-105.

Raimbourg, H., Toyoshima, T., Harima, Y., \& Kimura, G. (2008). Grain-size reduction mechanisms and rheological consequences in high temperature mylonites of Hidaka, japan. Earth and Planetary Science Letters, 267, 637-653.

Ravichandran, K. S., \& Seetharaman, V. (1993). Prediction of steady state creep behavior of two phase composites. Acta Materalia, 41(12), 3351-3361. 
Ricard, Y., \& Bercovici, D. (2009). A continuum theory of grain size evolution and damage. Journal of Geophysical Research, 114 , B01204. https://doi.org/10.1029/2007JB005491

Roberts, C. (2008). Grain growth and the Zener pinning phenomenon: A computational and experimental investigation (PhD thesis), Carnegie Mellon University.

Rozel, A., Ricard, Y., \& Bercovici, D. (2011). A thermodynamically self-consistent damage equation for grain size evolution during dynamic recrystallization. Geophysical Journal International, 184, 719-728. https://doi.org/10.1111/j.365-246x.2010.04875.x

Rutter, E. H., \& Brodie, K. H. (1988). The role of grain-size reduction in the rheological stratification of the lithosphere. Geologische Rundschau, 77(1), 295-308.

Rybacki, E., \& Dresen, G. (2000). Dislocation and diffusion creep of synthetic anorthite aggregates. Journal of Geophysical Research, 105(B11), 26,017-26,036.

Rybacki, E., \& Dresen, G. (2004). Deformation mechanism maps for feldspar rocks. Tectonophysics, 382, $173-187$.

Schubert, G., \& Yuen, D. A. (1978). Shear heating instability in the Earth's upper mantle. Tectonophysics, 50, 197-205.

Shimizu, I. (1998). Stress and temperature dependence of recrystallized grain size: A subgrain misorientation model. Geophysical Research Letters, 25(22), 4237-4240.

Shimizu, I. (2008). Theories and applicability of grain size piezometers: The role of dynamic recrystallization mechanisms. Journal of Structural Geology, 30, 899-917.

Slotemaker, A. K. (2006). Dynamic recrystallization and grain growth in olivine rocks (PhD thesis), Utrecht University.

Smith, C. S. (1948). Grains, phases, and interfaces: An interpretation of microstructure. Trans AIME, 175, 15-51.

Stipp, M., \& Tullis, J. (2003). The recristallised grain size piezometer for quartz. Geophysical Research Letters, $30(21)$, 2088. https://doi.org/10.1029/2003GL018444

Sullivan, W. A., Boyd, A. S., \& Monz, M. E. (2013). Strain localization in homogeneous granite near the brittle-ductile transition: A case study of the Kellyland Fault Zone, Maine, USA. Journal of Structural Geology, 56, 70-88.

Tasaka, M., Zimmerman, M. E., Kohlstedt, D. L., Stunitz, H., \& Heilbronner, R. (2017). Rheological weakening of olivine + orthopyroxene aggregates due to phase mixing, part 2: Microstructural development. Journal of Geophysical Research: Space Physics, 122, 7597-7612. https://doi.org/ 10.1002/2017jb014311

Ter Heege, J. H., De Bresser, J. H. P., \& Spiers, C. J. (2004). Composite flow laws for crystalline materials with log-normally distributed grain size: Theory and application to olivine. Journal of Structural Geology, 26, 1693-1705.

Tullis, T. E., Horowitz, F. G., \& Tullis, J. (1991). Flow laws of polyphase aggregates from end-member flow laws. Journal of Geophysical Research, 96(B5), 8081-8096.

Twiss, J. (1977). Robert. Theory and applicability of a recristallized grain-size paleopiezometer, Pure and Applied Geophysics, 115, 227-244.

Van der Wal, D., Chopra, P., Drury, M., \& Gerald, J. F. (1993). Relationship between dynamically recrystallization grain size and deformation conditions in experimentally deformed olivine rocks. Geophysical Research Letters, 20(14), 1479-1482.

Warren, J. M., \& Hirth, G. (2006). Grain size sensitive deformation mechanisms in naturally deformed peridotites. Earth and Planetary Science Letters, 248, 438-450.

Weertman, J. (1957). Steady-state creep through dislocation climb. Journal of applied physics, 28, 362-364.

White, S. H., Burrows, S. E., Carreras, J., Shaw, N. D., \& Humphreys, F. J. (1980). On mylonites in ductile shear zones. Journal of Structural Geology, 2(1/2), 175-187.

Wilks, K. R., \& Carter, N. L. (1990). Rheology of some continental lower crustal rocks. Tectonophysics, 182, 57-77. 